MANCHESTER

1824

†⿺

Economics

这突 Discussion Paper Series

E

\title{
The Performance of Lag Selection and Detrending Methods for HEGY Seasonal Unit Root Tests
}

Tomás del Barrio Castro

Denise R. Osborn

A.M. Robert Taylor

October 2012

Economics

School of Social Sciences

The University of Manchester

Manchester M13 9PL 


\title{
The Performance of Lag Selection and Detrending Methods for HEGY Seasonal Unit Root Tests*
}

\author{
Tomás del Barrio Castro \\ Department of Applied Economics \\ University of the Balearic Islands
}

\author{
Denise R. Osborn \\ Economics, School of Social Sciences \\ University of Manchester
}

\author{
A.M. Robert Taylor \\ School of Economics \\ University of Nottingham
}

October 2012

\begin{abstract}
This paper analyzes two key issues for the empirical implementation of parametric seasonal unit root tests, namely GLS versus OLS detrending and the selection of the lag augmentation polynomial. Through an extensive Monte Carlo analysis, the performance of a battery of lag selection techniques is analyzed, including a new extension of modified information criteria for the seasonal unit root context. All procedures are applied for both OLS and GLS detrending for a range of data generating processes, also including an examination of hybrid OLS-GLS detrending in conjunction with (seasonal) modified AIC lag selection. An application to quarterly US industrial production indices illustrates the practical implications of choices made.
\end{abstract}

Keywords: seasonal unit root; HEGY tests; data-based lag selection; OLS and GLS detrending. JEL codes: C22; C52.

\section{Introduction}

In their seminal paper, Hylleberg, Engle, Granger and Yoo (1990) [HEGY] develop seasonal unit root tests which enable separate regression-based $t$ - and $F$-tests to be conducted for unit roots at the zero, semi-annual and annual frequencies for quarterly data. Many subsequent papers build on this approach, including Ghysels, Lee and Noh (1994), who extend the analysis to consider joint tests for unit roots at the zero and all seasonal frequencies, and Smith, Taylor and del Barrio Castro (2009) who generalise the approach to the case of an arbitrary seasonal data frequency.

${ }^{*}$ Tomás del Barrio Castro gratefully acknowledges financial support from Ministerio de Educación y Ciencia ECO201123934. Address correspondence to: Robert Taylor, School of Economics, University of Nottingham, Nottingham, NG7 2RD, UK. E-mail: Robert.Taylor@nottingham.ac.uk 
The original HEGY analysis assumes that the time series under study follows a finite-order autoregressive (AR) process, with empirical researchers almost invariably taking the same stance when they employ these tests. However, the AR assumption contrasts with other literature concerned with seasonal time series. For example, seasonal adjustment is based largely on models that have important moving average (MA) components; see Cleveland and Tiao (1976) or Burridge and Wallis (1984). Although it has been widely conjectured that an AR approximation can be applied when conducting HEGY tests for an ARMA process (Taylor, 1997), the required theoretical justification has been provided only very recently by del Barrio Castro and Osborn (2011) and del Barrio Castro, Osborn and Taylor (2012) [COT]. More specifically, COT show that popular tests based on the HEGY approach remain valid for a general ARMA process, provided that the order of the AR lag augmentation polynomial increases in proportion with the sample size at a suitable rate.

Nevertheless, empirical practice requires matters to be taken a stage further, since data-dependent methods are used to select the lag augmentation polynomial. To our knowledge, there has been no systematic study of the performance of different lag selection methods in this context. The present paper fills this important gap, using Monte Carlo methods to explore the small sample performance of a variety of methods for determining the lags to be included in a HEGY test regression. In particular, we examine sequential test procedures similar to those employed by Hall (1994) and Ng and Perron (1995), seasonal variants of these as suggested by Rodrigues and Taylor (2004) and Beaulieu and Miron (1993), and methods based on information criteria including $A I C, B I C$ and a seasonal extension we develop for the modified information criteria $[M A I C, M B I C]$ of $\mathrm{Ng}$ and Perron (2001). Further, in addition to allowing for deterministic components in the HEGY regression, we explore whether or not seasonal generalised least squares (GLS) detrending, developed by Rodrigues and Taylor (2007) from the GLS approach of Elliot, Rothenberg and Stock (1996), improves results. In this last context, we also examine whether the approach of Perron and Qu (2007), whereby lag specification is undertaken via modified information criteria in an OLS context prior to unit root testing based on GLS detrending, improves the power of GLS tests for alternatives distant from the respective seasonal unit root null.

Our Monte Carlo analysis allows us to make recommendations about how seasonal unit root tests should be applied in practice. An empirical application to quarterly US industrial production series shows how decisions made about lag specification and detrending can influence the conclusions drawn about the presence of (seasonal) unit roots. Both of these decisions are seen to be important in practice.

The remainder of the paper is organised as follows. Section 2 outlines the seasonal model for quarterly data, defines the hypotheses of interest within that model, briefly reviews the augmented HEGY-type seasonal unit root tests and their limiting null distributions, then discusses lag selection and detrending methods. Section 3 presents our investigation of the finite sample performance of HEGY-type tests based on a variety of data-based lag selection methods for series driven by both MA and AR shocks. In addition to size and local power investigations of conventional ordinary least squares (OLS) detrending versus GLS detrending, this section examines power issues arising from OLS, GLS and hybrid OLS-GLS detrending for DGPs distant from the unit root null. The empirical application 
to US industrial production indices is the focus of section 4, while section 5 concludes. Throughout this paper we study HEGY-type tests at the quarterly data frequency, since the vast majority of empirical applications of seasonal unit root tests employ such data. However, the recommendations we make will also be useful in the context of other data frequencies.

\section{The Seasonal Unit Root Test Framework}

This section considers the model and hypotheses of interest, together with lag specification and detrending methods that may be employed when testing for the presence of unit roots in the seasonal context.

\subsection{The Seasonal Model and Unit Root Hypotheses}

Consider a univariate seasonal time-series process $\left\{x_{4 t+s}\right\}$ observed at the quarterly frequency from the data generating process (DGP):

$$
\begin{aligned}
x_{4 t+s} & =\mu_{4 t+s}+y_{4 t+s}, s=-3, \ldots, 0, t=1,2, \ldots, N \\
\alpha(L) y_{4 t+s} & =u_{4 t+s} \\
u_{4 t+s} & =\psi(L) \varepsilon_{4 t+s}
\end{aligned}
$$

where $\alpha(z):=1-\sum_{j=1}^{4} \alpha_{j}^{*} z^{j}$, is an $A R(4)$ polynomial in the conventional lag operator, $L$. The term $\mu_{4 t+s}:=\gamma^{\prime} Z_{4 t+s}$ in 2.1a is purely deterministic. The DGP of 2.1a to 2.1c can be generalised to any seasonal aspect $S$; see, for example, Smith, Taylor and del Barrio Castro (2009) or COT. Further, Smith et al. (2009) present a typology of six cases of interest for $\mu_{4 t+s}$, namely: no deterministic component; non-seasonal intercept; non-seasonal intercept and non-seasonal trend; seasonal intercepts; seasonal intercepts and non-seasonal trend, and seasonal intercepts and seasonal trends.

The error process $u_{4 t+s}$ in $2.1 \mathrm{c}$ is a linear process with $\psi(z):=1+\sum_{j=1}^{\infty} \psi_{j} z^{j}$. Following COT and Chang and Park (2002), the polynomial $\psi(z)$ is assumed to satisfy $\psi(z) \neq 0$ for all $|z| \leq 1$ and $\sum_{j=1}^{\infty}|j|^{\tau}\left|\psi_{j}\right|<\infty$ for some $\tau \geq 1$. Thus, $u_{4 t+s}$ admits the causal and invertible $\operatorname{ARMA}(p, q)$ representation, $\phi(L) u_{4 t+s}=\theta(L) \varepsilon_{4 t+s}$, such that all the roots of $\phi(z):=1-\sum_{i=1}^{p} \phi_{p} z^{i}$ and $\theta(z):=1-\sum_{i=1}^{q} \theta_{i} z^{i}$ lie strictly outside the unit circle. The martingale difference innovation sequence $\left(\varepsilon_{4 t+s}, \mathcal{F}_{4 t+s}\right)$ with filtration $\left(\mathcal{F}_{4 t+s}\right)$ satisfies $E\left(\varepsilon_{4 t+s}^{2} \mid \mathcal{F}_{4 t+s-1}\right)=\sigma^{2}$ and $E\left|\varepsilon_{4 t+s}\right|^{r}<K$ with $r \geq 4$, where $K$ is some constant depending only upon $r$. The homoskedasticity assumed here can be weakened to allow conditional heteroscedasticity, at the cost of a stronger being required on the lag truncation in the HEGY test; see the discussion in COT and subsection 2.2 below.

Our focus is on tests for seasonal unit roots in $\alpha(L)$ of $2.1 \mathrm{~b}$; i.e., the null hypothesis of interest is

$$
H_{0}: \alpha(L)=1-L^{4}=: \Delta_{4}
$$

Under $H_{0}$ of 2.2 , the DGP for $\left\{x_{4 t+s}\right\}$ is often referred to as a seasonally integrated process. Factorizing the $\operatorname{AR}(4)$ polynomial $\alpha(L)$ as $\alpha(L)=\prod_{j=0}^{2} \omega_{j}(L)$, where $\omega_{0}(L):=\left(1-\alpha_{0} L\right)$ associates the 
parameter $\alpha_{0}$ with the zero frequency, $\omega_{1}(L):=\left[1+2 \beta_{1} L+\left(\alpha_{1}^{2}+\beta_{1}^{2}\right) L^{2}\right]$ corresponds to the annual seasonal frequency $\pi / 2$, with associated parameters $\alpha_{1}$ and $\beta_{1}$, and $\omega_{2}(L):=\left(1+\alpha_{2} L\right)$ associates the parameter $\alpha_{2}$ with the Nyquist (or semi-annual) frequency $\pi$. Consequently $H_{0}$ of $(2.2)$ may be commensurately partitioned as $H_{0}=\cap_{j=0}^{2} H_{0, j}$, where

$$
H_{0, i}: \alpha_{i}=1, \quad i=0,2, \text { and } H_{0,1}: \alpha_{1}=1, \beta_{1}=0 .
$$

The hypothesis $H_{0,0}$ corresponds to a unit root at the zero frequency, $H_{0,2}$ yields a unit root at the semi-annual frequency and a pair of complex conjugate unit roots at frequency $\pi / 2$ is obtained under $H_{0,1}$. The alternative hypothesis $H_{1}$ is of stationarity at one or more of the zero or seasonal frequencies; that is, $H_{1}=\cup_{j=0}^{2} H_{1, j}$, where

$$
H_{1, i}: \alpha_{i}<1, \quad i=0,2 \text {, and } H_{1,1}: \alpha_{1}^{2}+\beta_{1}^{2}<1
$$

Consequently, the maintained hypothesis $H_{0} \cup H_{1}$ excludes all unit roots, except for a possible single unit root at each of the zero and Nyquist frequencies and a pair of complex conjugate unit roots at the harmonic seasonal frequency $\pi / 2$. Explosive roots in $\alpha(L)$ are also excluded.

\subsection{Augmented HEGY Tests}

Under the assumption that the DGP of 2.1a) and 2.1b is of a purely AR form, HEGY develop a seasonal unit root test regression for quarterly data 1 , which can be written as

$$
\Delta_{4} x_{4 t+s}=\pi_{0} x_{0,4 t+s}+\pi_{2} x_{2,4 t+s}+\pi_{1} x_{1,4 t+s}+\pi_{1}^{*} x_{1,4 t+s}^{*}+\mu_{4 t+s}^{*}+\sum_{j=1}^{k} d_{j} \Delta_{4} x_{4 t+s-j}+e_{4 t+s}^{k}
$$

where

$$
\begin{aligned}
& x_{0,4 t+s}:=\sum_{j=0}^{3} x_{4 t+s-j-1}, \quad x_{2,4 t+s}:=\sum_{j=0}^{3}(-1)^{j+1} x_{4 t+s-j-1}, \\
& x_{1,4 t+s}:=-x_{4 t+s-2}+x_{4 t+s-4}, \quad x_{1,4 t+s}^{*}:=-x_{4 t+s-1}+x_{4 t+s-3}
\end{aligned}
$$

and $\mu_{4 t+s}^{*}:=\gamma^{* \prime} Z_{4 t+s}$, so that the deterministic component of $(2.1 \mathrm{a})$ is appropriately specified. It is important to notice, as shown in Smith et al. (2009), that the inclusion of seasonal intercepts renders the resulting unit root tests similar with respect to the initial conditions $y_{1-S}, \ldots, y_{0}$. The regression 2.5 is estimated by OLS over observations $4 t+s=k+1, \ldots, T$. The superscript $k$ in $e_{4 t+s}^{k}$ indicates that this process depends on the order of augmentation applied, since our analysis does not assume either that the test regression employs the true AR order nor, indeed, that the disturbances in the DGP $2.1 \mathrm{c})$ are of an AR form.

Tests of the hypotheses (2.3) for the presence (or otherwise) of a unit root at the zero and Nyquist frequencies may be conducted using conventional lower tailed regression $t$-statistics, denoted $t_{0}$ and

\footnotetext{
${ }^{1}$ The test regression and HEGY-type tests corresponding to 2.5 for a general seasonal aspect $S$ are presented by Smith and Taylor (1999) and COT, among others.
} 
$t_{2}$, for the exclusion of $x_{0,4 t+s}$ and $x_{2,4 t+s}$, respectively, from 2.5 . Similarly, the hypothesis of a pair of complex unit roots at the annual seasonal frequency may be examined using the lower-tailed $t_{1}$ and two-tailed $t_{1}^{*}$ regression $t$-statistics for the exclusion of $x_{1,4 t+s}$ and $x_{1,4 t+s}^{*}$, respectively, or by the (upper-tailed) regression $F$-test, denoted $F_{1}$, for the exclusion of both $x_{1,4 t+s}$ and $x_{1,4 t+s}^{*}$ from 2.5. Ghysels, Lee and Noh (1994) and Smith et al. (2009) also consider joint frequency (uppertail) regression $F$-tests from (2.5), namely $F_{12}$ for the exclusion of $x_{2,4 t+s}$, together with $x_{1,4 t+s}$ and $x_{1,4 t+s}^{*}$, and $F_{012}$ for the exclusion of $x_{0,4 t+s}, x_{2,4 t+s}$, and $x_{1,4 t+s}$ and $x_{1,4 t+s}^{*}$. The former tests the null hypothesis of unit roots at all seasonal frequencies, while the latter tests the overall null, namely $H_{0}$ of 2.2 .

Our analysis studies strategies to specify the AR augmentation order $k$ in 2.5 such that asymptotically valid and empirically reliable seasonal unit root tests can be applied. As discussed by COT (see, in particular, their Remark 9), data-based augmentation lag selection procedures are asymptotically justified if the truncation lag is allowed to increase with the sample size, such that $k \rightarrow \infty$ with $k=o\left([T / \log T]^{1 / 2}\right)$ as $T \rightarrow \infty$ when the innovations in the DGP $2.1 \mathrm{c}$ are conditionally homoskedastic. It may be noted that the faster rate $k=o\left(T^{1 / 2}\right)$ is sufficient for the asymptotic validity of the HEGY tests, but this does not guarantee the consistency of the estimators of the coefficients, $d_{j}$, $j=1, \ldots, k$, on the lagged dependent variables; see COT and the analogous discussion in Chang and Park (2002) for the conventional ADF test. However, as also noted by COT (Remark 9), a slower rate of $k=o\left(T^{1 / 3}\right)$ rate is required for data-based lag selection in 2.5 when conditional heteroscedasticity is permitted in the innovations of $2.1 \mathrm{c}$.

Hence, provided that the maximum $k$ considered in relation to the sample size $T$ is appropriate in the context of the innovation properties, empirical lag selection procedures can be applied and will result in asymptotic null distributions for the HEGY test statistics identical to those for a DGP where $u_{4 t+s}$ in $2.1 \mathrm{~b}$ is serially uncorrelated and conditionally homoscedastic. In other words, the limiting null distributions of the $t_{0}, t_{2}, F_{1}, F_{12}$ and $F_{012}$ statistics from 2.5) are then invariant to the serial correlation nuisance parameters $\left\{\psi_{j}\right\}_{j=1}^{\infty}$ which characterise the serial dependence of $u_{4 t+s}$ in the DGP (2.1c). Consequently, for the case of quarterly data the critical values given in Smith and Taylor (1998, pp.279-280) can be used, with analogous results applying for other values of $S$. As in Burridge and Taylor (2001), the regression $t$-statistics for the exclusion of $x_{1,4 t+s}$ and $x_{1,4 t+s}^{*}$ depend on the form of the serial correlation in the DGP and these should not be used in any case where augmentation is required.

\subsection{Lag Selection Methods}

Implementation of the HEGY test requires the augmentation order $k$ of 2.5 to be specified. We consider a variety of data-based methods for this purpose, including both information criteria and testing strategies. In relation to the former, we employ the standard information criteria, $A I C$ and $B I C$. Further, following $\mathrm{Ng}$ and Perron (2001), modified $A I C$ and $B I C$ [MAIC and $M B I C$, respectively] are developed for the seasonal unit root context and included in our Monte Carlo comparisons. 


\subsubsection{Information Criteria}

Under the zero frequency unit root null hypothesis of the ADF test, $\mathrm{Ng}$ and Perron (2001) extend the usual information criteria to incorporate the distance from the unit root null; they argue that their additional term is particularly important to account for the presence of a negatively autocorrelated MA process under the null. This leads them to consider the class of modified information criteria which select $k$ to minimise

$$
M I C(k):=\ln \left(\hat{\sigma}_{k}^{2}\right)+\frac{C_{T}\left[\tau_{T}(k)+k\right]}{T-k_{\max }}
$$

where $\hat{\sigma}_{k}^{2}:=R S S_{k} /\left(T-k_{\max }\right)$ in which $R S S_{k}$ is the residual sum of squares obtained from the unit root test regression augmented with $k$ lags of the dependent variable and $k_{\max }$ is the maximum value of $k$ considered, $C_{T}$ is defined by the specific criterion $\left(C_{T}:=2\right.$ for $M A I C$ and $C_{T}:=\ln \left(T-k_{\max }\right)$ for $M B I C)$, and $\tau_{T}(k)$ is sample dependent. The standard information criteria $A I C$ and $B I C$ set $\tau_{T}(k)=0$.

The approach of Ng and Perron (2001, pp.1528-1529) is based on Kulback distance as embedded in $A I C$. To extend this to the seasonal unit root null hypothesis of $(2.2)$, consider the special case of the HEGY regression (2.5) with no determinsitic component $\left(\mu_{4 t+s}^{*}=0\right)$ and define the vector of regressors as $X_{4 s+t}:=\left[x_{0,4 t+s}, x_{2,4 t+s}, x_{1,4 t+s}, x_{1,4 t+s}^{*}, \Delta_{4} x_{4 t+s-1}, \ldots, \Delta_{4} x_{4 t+s-k}\right]^{\prime}$ with corresponding coefficient vector $\delta(k):=\left[\pi_{0}, \pi_{2}, \pi_{1}, \pi_{1}^{*}, d_{1}, \ldots, d_{k}\right]^{\prime}$. Under the overall null hypothesis of $(2.2)$, this latter vector is $\delta^{0}(k):=\left[0,0,0,0, d_{1}, \ldots, d_{k}\right]^{\prime}$. An empirical measure of the Kulback distance of the parametric model, with estimated coefficient vector $\widehat{\delta}(k)$, from the true model under the null hypothesis is given by

$$
\Psi_{T}(k):=\left(1 / \hat{\sigma}_{k}^{2}\right)\left(\widehat{\delta}(k)-\delta^{0}(k)\right)^{\prime} \sum_{t} \sum_{s} X_{4 t+s} X_{4 t+s}^{\prime}\left(\widehat{\delta}(k)-\delta^{0}(k)\right)
$$

where the double sum in (2.8) corresponds to observations $k_{\max }+1, \ldots, T$ used for estimation. Noting the asymptotic orthogonality between the regressors that are integrated and those that are stationary under the null hypothesis and also the mutual (asymptotic) orthogonality of the regressors $x_{j, 4 t+s}$, $j=0,1,2$, and $x_{1,4 t+s}^{*}$ in 2.5 , leads to $\Psi_{T}(k)=\tau_{T}(k)+\chi_{k}^{2}+o_{p}(1)$, with

$$
\tau_{T}(k):=\left(\hat{\sigma}_{k}^{2}\right)^{-1}\left(\sum_{j=0}^{2}\left[\hat{\pi}_{j}^{2} \sum_{t} \sum_{s}\left(x_{j, 4 t+s}\right)^{2}\right]+\left[\left(\hat{\pi}_{1}^{*}\right)^{2} \sum_{t} \sum_{s}\left(x_{1,4 t+s}^{*}\right)^{2}\right]\right)
$$

where the chi-square variable with $k$ degrees of freedom, $\chi_{k}^{2}$, is asymptotically independent of $\tau_{T}(k)$. Analogously to $\mathrm{Ng}$ and Perron (2001), the criterion MAIC for the quarterly augmented HEGY seasonal unit root test regression replaces $k$ (namely the expected value of $\chi_{k}^{2}$ ), as used in the standard version of $A I C$, by $\tau_{T}(k)+k$ with $\tau_{T}(k)$ defined by $(2.9)$. This $\tau_{T}(k)$ can also be employed in 2.7 to define $M B I C$.

In practice, the test regression typically includes a deterministic component $\mu_{4 t+s}^{*}$, which needs to be removed when computing (2.9). Therefore, define $\widehat{x}_{4 t+s}:=x_{4 t+s}-\left(\widehat{\gamma}_{O L S}^{*}\right)^{\prime} Z_{4 t+s}$ in which $\widehat{\gamma}_{O L S}^{*}$ is the estimated coefficient vector in a regression of $x_{4 t+s}$ on $Z_{4 t+s}$. Then $\widehat{x}_{j, 4 t+s}, j=0,1,2$ and $\widehat{x}_{1,4 t+s}^{*}$, 
obtained by applying the HEGY transformations 2.6a and 2.6b), respectively, to the detrended observations, are employed for the computation of $(2.9)$ when used in the context of the HEGY rgression 2.5.

In the light of the finding of Hall (1994) that using an information criterion to select the maximum lag $k$ over $k=0,1, \ldots, k_{\max }$ may lead to size distortions in cases (such as the seasonal context) where there are "gaps" in the $d_{j}$ coefficients, we propose an alternative sequential method (labelled in the tables as $S A I C, S B I C, S M A I C, S M B I C)$. This starts by computing the relevant criterion for $k=k_{\max }$, with the value then computed with each individual lag $1, \ldots, k_{\max }$ deleted, one-by-one. If the criterion is improved by dropping any lag, the single lag that has the least effect on the criterion is removed from 2.5), and the procedure is repeated from this new specification. This procedure stops when no improvement in the criterion results from deleting any additional individual lag.

\subsubsection{Sequential Testing}

In addition to information criteria procedures, sequential methods based on the significance of individual lag coefficients are also examined, using $5 \%$ and $10 \%$ critical values from the standard normal distribution. Following Ng and Perron (1995) and Hall (1994), one procedure "tests down" from $k_{\max }$ to determine the maximum lag $k$ to be employed (with no gaps); these methods are denoted as $t-s q(5 \%)$ and $t-s q(10 \%)$, respectively ${ }^{2}$, Results are also reported for the approach proposed by Beaulieu and Miron (1993), where (2.5) is estimated for given maximum lag order $k_{\max }$ and all lagged values with coefficients individually insignificant at the $5 \%$ or $10 \%$ level are deleted in a single step; we denote these as $t$-bm(5\%) and $t$-bm(10\%). Finally, the sequential method used in Rodrigues and Taylor (2004) is employed, where at each stage the least significant of any lagged dependent variable coefficient is deleted, until all remaining coefficients are significant $[t-r t(5 \%)$ and $t-r t(10 \%)]$.

\subsection{Seasonal GLS Detrending}

The HEGY approach of 2.5 includes any deterministic terms required in the test regression itself. However, in the context of conventional zero frequency unit root tests, Elliott et al. (1996) show that important power gains can result if prior detrending is undertaken to purge the series of the deterministic component under local to unit root asymptotics, by application of GLS detrending. The unit root test regression is then estimated using the detrended data without any deterministic component. Indeed, the modified information criteria of $\mathrm{Ng}$ and Perron (2001) were initially proposed in this context.

Rodrigues and Taylor (2007) study optimal tests for seasonal unit roots, with these giving rise to GLS-detrended tests which extend the zero frequency tests of Elliott et al. (1996) and also those of Gregoir (2006) for a pair of complex unit roots. GLS detrending (for the quarterly case) is achieved by

\footnotetext{
${ }^{2}$ For this procedure, and also those suggested by Beaulieu and Miron (1993) and Rodrigues and Taylor (2004a), results were also obtained for a significance level of $15 \%$. These are excluded to conserve space, but exhibit qualitatively similar patterns to the corresponding $10 \%$ ones.
} 
regressing the seasonal quasi-difference $\mathbf{x}_{\bar{c}}$ vector on the quasi-difference $\mathbf{Z}_{\bar{c}}$ matrix for the deterministic component, where these are defined using

$$
\begin{gathered}
\mathbf{x}_{\bar{c}}:=\quad\left[x_{1}, x_{2}-\alpha_{1}^{\bar{c}} x_{1}, x_{3}-\alpha_{1}^{\bar{c}} x_{2}-\alpha_{2}^{\bar{c}} x_{1}, \ldots, x_{4}-\alpha_{1}^{\bar{c}} x_{3}-\alpha_{2}^{\bar{c}} x_{2}-\alpha_{3}^{\bar{c}} x_{1}, \bar{\Delta}_{4} x_{5}, \ldots, \bar{\Delta}_{4} x_{T}\right]^{\prime} \\
\mathbf{Z}_{\bar{c}}:=\quad\left[Z_{1}, Z_{2}-\alpha_{1}^{\bar{c}} Z_{1}, Z_{3}-\alpha_{1}^{\bar{c}} Z_{2}-\alpha_{2}^{\bar{c}} Z_{1}, Z_{4}-\alpha_{1}^{\bar{c}} Z_{3}-\alpha_{2}^{\bar{c}} Z_{2}-\alpha_{3}^{\bar{c}} Z_{1},\right. \\
\left.\bar{\Delta}_{4} Z_{5}, \ldots, \bar{\Delta}_{4} Z_{T}^{\prime}\right]
\end{gathered}
$$

where

$$
\bar{\Delta}_{4}:=\left[1-\left(1-\frac{\bar{c}_{0}}{T}\right) L\right]\left[1+\left(1-\frac{\bar{c}_{2}}{T}\right) L\right]\left[1+\left(1-\frac{\bar{c}_{1}}{T}\right)^{2} L^{2}\right]=\left(1-\sum_{j=1}^{4} \alpha_{j}^{\bar{c}} L^{j}\right) .
$$

When $\mu_{4 t+s}^{*}$ allows (constant) seasonal means, the analyses of Elliott et al. (1996) and Gregoir (2006) for unit root tests at the $5 \%$ level lead Rodrigues and Taylor (2007) to propose values of $\bar{c}_{0}=\bar{c}_{2}=7$ and $\bar{c}_{1}=3.75$, while trending seasonal means in $\mu_{4 t+s}^{*}$ (so that $Z_{4 t+4}$ contains both seasonally varying intercepts and trends) lead to the recommendations $\bar{c}_{0}=\bar{c}_{2}=13.5$ and $\bar{c}_{1}=8.65$. Other cases are also possible, such as seasonal intercepts but a common trend over quarters $\left(\bar{c}_{0}=13.5, \bar{c}_{2}=7, \bar{c}_{1}=3.75\right)$.

Denoting as $\widetilde{\gamma}_{G L S}^{*}$ the estimated coefficient vector from the regression using the transformed data of 2.10, the GLS detrended series is then given by $\widetilde{x}_{4 t+s}:=x_{4 t+s}-\left(\widetilde{\gamma}_{G L S}^{*}\right)^{\prime} Z_{4 t+s}$. For an augmentation order $k$, GLS detrended HEGY tests are applied using the test regression

$$
\Delta_{4} \widetilde{x}_{4 t+s}=\pi_{0} \widetilde{x}_{0,4 t+s}+\pi_{2} \widetilde{x}_{2,4 t+s}+\pi_{1} \widetilde{x}_{1,4 t+s}+\pi_{1}^{*} \widetilde{x}_{1,4 t+s}^{*}+\sum_{j=1}^{k} d_{j} \Delta_{4} \widetilde{x}_{4 t+s-j}+\widetilde{e}_{4 t+s}^{k}
$$

where $\widetilde{x}_{0,4 t+s}, \widetilde{x}_{2,4 t+s}, \widetilde{x}_{1,4 t+s}$ and $\widetilde{x}_{1,4 t+s}^{*}$ are defined analogously to $\left.2.6 \mathrm{a}\right)$ and $\left.2.6 \mathrm{~b}\right)$, as appropriate. Rodrigues and Taylor (2007) present critical values for the tests of interest, namely for $t_{0}, t_{2}, F_{1}, F_{12}$ and $F_{012}$. This methodology is employed to deliver seasonal unit root tests with GLS detrending, with the lag specification for $k$ made in the context of (2.11) using all the information criteria approaches and testing down strategies as discussed in the preceding subsection. For the modified information criteria, and analogously to $\mathrm{Ng}$ and Perron (2001), the additional penalty term $\tau_{T}(k)$ of 2.9 is computed using GLS detrended data.

However, Perron and Qu (2007) note that employing GLS detrending with lags specified by modified information criteria can result in conventional zero frequency unit root tests with poor power for alternatives that are not close to the null. To counter this, they recommend that although testing be conducted using the GLS detrended data, the augmentation order $k$ be specified using OLS detrended series. We investigate this methodology for the modified criteria $M A I C, M B I C, S M A I C$ and $S M B I C$. More explicitly, lags are specified using each criterion in a regression entirely analogous to 2.11, except that OLS detrended data $\widehat{x}_{4 t+s}=x_{4 t+s}-\left(\widehat{\gamma}_{O L S}^{*}\right)^{\prime} Z_{4 t+s}$ are employed in place of $\widetilde{x}_{4 t+s}$ and with $\widehat{x}_{0,4 t+s}, \widehat{x}_{2,4 t+s}, \widehat{x}_{1,4 t+s}$ and $\widehat{x}_{1,4 t+s}^{*}$ again defined in a manner corresponding to 2.6a) and (2.6b), as appropriate, with OLS-detrended data also used to define the additional penalty $\tau_{T}(k)$ of (2.9). Once $k$ is specified (together with any "gaps" for $S M A I C$ and $S M B I C$ ), the GLS transformed 
variables of 2.10 are used to compute the unit root test statistics in the context of 2.11. Following Perron and Qu (2007), the discussion below refers to this as OLS-GLS detrending; unless stated otherwise, GLS detrending employs the Rodrigues and Taylor (2007) procedure with lag specification made in the context of 2.11 .

\section{Finite Sample Comparison}

After setting out our Monte Carlo methodology, the following subsections discuss the results for the finite sample size and power delivered by the seasonal unit root test procedures of section 2 also drawing out implications for empirical analysis.

\subsection{Methodology}

Data are generated according to the mode $3^{3}$

$$
x_{4 t+s}=(1-c / N) x_{4(t-1)+s}+u_{4 t+s}=\alpha_{4} x_{4(t-1)+s}+u_{4 t+s}, \quad s=-3, \ldots, 0, \quad t=1, \ldots, N
$$

with initial conditions set to zero for samples with $N=60,100(T=240$ and 400$)$. Size results are obtained with $c=0$ and size-adjusted local power with $c=5,10,20$; as discussed in Rodrigues and Taylor (2004), the process is stationary at both the zero and each seasonal frequency when $c>0$. In addition to white noise innovations, we consider serial correlation in $u_{4 t+s}$ of both MA and AR forms, with these being special cases of

$$
u_{4 t+s}=(1-\theta L)\left(1-\Theta L^{4}\right) \varepsilon_{4 t+s}
$$

and

$$
\left(1-\Phi L^{4}\right) u_{4 t+s}=\varepsilon_{4 t+s}
$$

respectively, where $\varepsilon_{4 t+s} \sim N I D(0,1)$. For $(3.2)$, our Monte Carlo investigation examines $\theta=-0.8$ with $\Theta=0$ and $\Theta= \pm 0.5$ with $\theta=0$. For $(3.3)$, we consider $\Phi= \pm 0.5$. In addition to size-adjusted local power, a fuller investigation of the power of GLS versus OLS detrending, including the OLS-GLS procedure of Perron and $\mathrm{Qu}$ (2007), is undertaken for lag specification methods based on MAIC using white noise and seasonal MA innovations.

Results are reported for the $t_{0}, t_{2}, F_{1}, F_{12}$ and $F_{012}$ test statistics, with these obtained from both the quarterly HEGY regression of (2.5) and using the seasonal GLS detrended test regression of (2.11), with these labelled as $O L S$ and $G L S$, respectively, in the tables. In both cases, the deterministic component allows seasonal intercepts and a zero frequency trend, as commonly applied in empirical analyses for seasonal data. Lag selection is based on the methodologies outlined in subsection 2.3 . As discussed in subsection 2.4 , the results using the modified information criteria after GLS detrending are

\footnotetext{
${ }^{3}$ We are grateful to a referee who suggested the inclusion of some DGPs where unit roots were present at some but not all (zero and seasonal) frequencies of interest. However, an extension of the analysis here found that DGPs with local departures from the unit root null at some frequencies yielded very similar results overall to those reported.
} 
presented both for lag selection made in the test regression 2.11) and in the corresponding regression for OLS detrended data; the latter is the OLS-GLS procedure and is indicated in the tables by the suffix $P Q$. To conserve space, results are not reported for the sequential $B I C$ procedures (namely, $S B I C, S M B I C$ and $S M B I C \_P Q$ ), but these reveal similar patterns to the corresponding sequential $A I C$ procedures shown.

The maximum initial lag is set as $k_{\max }:=\left\lfloor\ell(4 N / 100)^{1 / 4}\right\rfloor$ with $\ell=4$ and $\ell=12$, as employed by Schwert (1989) and others. When applied for increasing $T$, this rate satisfies $k=o\left([T / \log T]^{1 / 2}\right)$, which is discussed in subsection 2.2 as being sufficient to yield asymptotically valid data-based lag selection for testing seasonal unit roots in the HEGY test regression when the DGP innovation process is conditionally homoscedastic, as in our case $4^{4}$ In practice, our focus is on $k_{\text {max }}$ defined with $\ell=12$, which is also used by $\mathrm{Ng}$ and Perron (2001) for the modified information criteria. For the realistic case in applied work of $N=60$ years of data, $\ell=12$ implies the use of a maximum augmentation lag of 14 quarters, whereas $\ell=4$ leads to 4 lags being considered.

All unit root tests employ a nominal $5 \%$ significance level, using asymptotic critical values $5^{5}$ Results for empirical size $(c=0)$ are reported in Tables 1 to 3 (and discussed in subsection 3.2), with corresponding results for size-adjusted local power $(c=5,10$ and 20) in Tables 4 to 6 (discussed in subsection 3.3. These employ the typical postwar sample size of $N=60$ years of data, which is also effectively that used in the empirical application of section 4 ; analogous results for $N=100$ are in the Appendix. The results in all tables are based on 5000 replications. Finally, subsection 3.4 focuses on how detrending options (OLS, GLS and OLS-GLS) perform in power terms over the range of values of $c$ from 0 to $N$ when $M A I C$ is employed for lag specification.

\subsection{Size Properties}

Although the DGP employed for Table 1 is a seasonal random walk with IID innovations, and hence no lag augmentation is required, the results show a number of interesting characteristics. Firstly, the parameterisations resulting from the use of modified information criteria (that is, $M A I C, M B I C$ and $S M A I C$ ) lead to under-sized tests in this case when applied in the HEGY test regression (2.5), with the sequential $S M A I C$ being particularly poorly sized with $\ell=12$. On the other hand, conventional $A I C$ and $B I C$ perform well. Secondly, the use of individual $t$-ratios to specify the lag length results in good size with $\ell=4$ in (2.5), but over-sizing in the more highly parameterised models resulting from $\ell=12$. Thirdly, GLS detrended statistics are often modestly over-sized, although under-sizing results when the sequential modified information criteria are applied with $\ell=12$; compared with GLS detrending, the OLS-GLS (or $P Q$ ) procedure of Perron and Qu (2007) has effectively no impact on size for the modified information criteria methods in Table 1. Fourthly, applying hypothesis tests based on

\footnotetext{
${ }^{4}$ Applied in the context of increasing sample size, this $k_{\max }$ satisfies $k=o\left(T^{1 / 3}\right)$ and hence also yields valid asymptotic inference in 2.5 when the innovation process is conditionally heteroscedastic. Although we experimented with a variety of non-IID martingale difference specifications for $\varepsilon_{4 t+s}$ in the context of the conventional HEGY test regression, the results were almost identical to those reported.

${ }^{5}$ These critical values were obtained by direct simulation using 100, 000 replications and $T=2000$.
} 
$t$-ratios for lag selection works better in the original HEGY regression than for GLS detrending, with the empirical size for the overall $F_{012}$ test in the latter being around twice the nominal size. Finally, as anticipated, and although $k_{\max }$ increases with $N$, empirical size typically improves when the larger sample is employed. This applies not only for the seasonal random walk (compare Appendix Table A.1 with Table 1), but also for the vast majority of other comparisons across $N=60$ and $N=100$, for a given DGP and lag selection method.

Subsequent size results are presented only for $\ell=12$. Not surprisingly, $\ell=4$ results in better size than those shown when the true process is an autoregression of order less than $k_{\max }$, but can perform very poorly when the DGP has an MA form or when $k_{\max }$ under-specifies the true AR order.

Table 2 examines MA disturbance processes. A positively autocorrelated MA(1) with coefficient $\theta=-0.8$, considered in Panel A, is fairly close to cancellation with the AR unit root -1 , hence distorting inference at the Nyquist frequency $\left(t_{2}\right.$, together with $F_{12}$ and $\left.F_{012}\right)$. Indeed, the use of BIC leads to a rejection probability of 50 percent at this frequency with both trending options. This nearcancellation is the situation for which modified criteria are designed and since higher augmentation improves the approximation to this process, $M A I C$ performs relatively well at the Nyquist frequency and sequential lag selection $(S M A I C)$ further improves this performance for the HEGY test regression (2.5), with empirical sizes of 0.072 and 0.048, respectively, in Table 2 (Panel A). Although $M A I C$ and $S M A I C$ also perform better than other lag selection methods for $t_{2}$ with GLS or OLS-GLS detrending, the empirical sizes are nevertheless more than double their nominal sizes. At other frequencies, tests based on modified information criteria are under-sized in the HEGY (OLS detrending) approach, and this is sometimes substantial (note especially the empirical size of 0.005 for $t_{0}$ using $S M A I C$ ). However, the size for $t_{0}$ and $F_{1}$ is improved with GLS or OLS-GLS detrending. Although the use of coefficient tests for lag selection also yield quite good size for the unit root tests at the zero and annual frequencies in the HEGY regression, they are very substantially over-sized for the Nyquist frequency test, with $t-s q(10 \%)$ being the best of this group when used in 2.5). Performances of the tests at each of these seasonal frequencies is reflected in the sizes of the joint tests $F_{12}$ and $F_{012}$, with those using $M A I C / M B I C$ being well-sized in the HEGY regression due to off-setting under- and over-sizing of the individual frequency tests 6 .

Recognising that $\left(1-0.5 L^{4}\right)=(1-0.84 L)(1+0.84 L)\left(1+0.71 L^{2}\right)$, the patterns implied by nearcancellation for the simple MA(1) process of Panel A carry over to the seasonal MA with $\Theta=0.5$ in Panel B of Table 2, where tests at all frequencies are prone to over-sizing and the greatest distortions apply when $B I C$ is used. The most reliable size overall is obtained using MAIC in the HEGY regression and $S M A I C$ with GLS detrending, with the former often having the best size performance for tests at individual frequencies and the latter for the overall joint statistic $F_{012}$. It is also notable

\footnotetext{
${ }^{6}$ We also investigated the performance of these lag selection criteria in the context of the HEGY test regression 2.5 for the MA(1) case of $\theta=0.8$, which interchanges the roles of the zero and Nyquist frequencies. As anticipated, this leads to over-sizing for $t_{0}$, as found by Hall (1994) and $\mathrm{Ng}$ and Perron (2001) for the Dickey-Fuller test. Analogous results were also found for the MA(2) $u_{4 t+s}=\left(1-0.64 L^{2}\right) \varepsilon_{4 t+s}$, where near-cancellation applies at the Nyquist frequency and hence the over-sizing relates to $F_{1}$.
} 
that, compared with GLS detrending, the $P Q$ (that is, OLS-GLS) version can result in a small deterioration in size performance for the modified information criteria in Panel B. The seasonal MA with $\Theta=-0.5$ in Panel $\mathrm{C}$ of Table 2 does not approximate any AR unit root in the DGP, and the patterns of Panel B of Table 1 largely carry over to this case. Nevertheless, compared with the white noise disturbances in Table 1 (Panel B), size distortions are typically increased for the seasonal MA with $\Theta=-0.5$. This applies particularly for the information criteria methods, with $M B I C$ or $S M A I C$ lag selection combined with OLS detrending leading to markedly under-sized tests for this seasonal MA process. Perhaps surprisingly, across Panels B and C of Table 2, methods that allow elimination of intermediate lags in the augmentation polynomial through hypothesis tests $(t-b m$ and $t-r t$ ) always lead to poorer size that the corresponding $t$-sq procedure that has no gaps, despite the implied AR approximation having a seasonal form. Similarly, it is noteworthy that although $S A I C$ improves on $A I C$ when $\Theta=0.5$, this sequential method does not have any evident size advantages when $\Theta=-0.5$. Further, such lag elimination can lead to very poor size for $S M A I C$, as already noted.

The seasonal AR processes of Table 3 provide further evidence that intermediate lag elimination can increase size distortions for tests, even when the true AR polynomials of (3.3) have some intermediate zero coefficients. This applies across $S A I C, S M A I C, t-b m$ and $t-r t$ and for both panels of the table, in comparison with the corresponding procedures with no such intermediate lag elimination and irrespective of the form of detrending. Indeed, the general patterns of results from Panel C of Table 2 are largely reproduced in the panels of Table 3, but (not surprisingly) with less marked size distortions when the DGP is AR in form.

Finally, by comparing empirical size for $N=60$ in Table 2 with the corresponding case with $N=100$ in Appendix Table A.2, it is evident that the larger sample size reduces the extent of over-sizing in the former, but under-sizing is overall only modestly improved (compare, for example, $S M A I C$ in Panel C across these tables). It is also worth remarking that the often substantial undersizing shown by $S M A I C$ in Table 3, especially when used in the HEGY regression, largely carries over when the sample size increases in Appendix Table A.3. Otherwise, however, empirical size is reliable for these seasonal AR processes when $N=100$ years of data are available.

\subsection{Size-Adjusted Local Power}

Tables 4 to 6 mirror Tables 1 to 3, but now consider size-adjusted local power. The DGPs considered with $N=60$, the values of $c=5,10$ and 20 correspond to processes with seasonal AR coefficients $\alpha_{4}=0.92,0.83$ and 0.67 , respectively, in (3.1). Note that, due to space constraints, some methods included in the size comparisons of earlier tables are now omitted. Specifically, under GLS detrending, the tables show power for $M B I C$ using only the $P Q$ variant (namely the OLS-GLS procedure), since this yields typically more powerful tests than lag specification in the GLS regression itself, as illustrated in Tables 4-6 by $M A I C$ and $S M A I C$. Further, the impact on power of using a 5 percent significance level in testing down is indicated by results for $t-s q(5 \%)$ compared with $t$-sq $(10 \%)$, with power for 
other sequential methods included only for the 10 percent level 7

A glance at Table 4 confirms that, when the innovations in (3.1) are white noise, GLS detrending delivers substantial power gains against near-integrated processes (with $c=5,10$ ) compared with the usual practice of accounting for deterministic components by including these in the HEGY regression 2.5. Across all methods, lower power is achieved for $t_{0}$ than $t_{2}$ due to the tests allowing for a zero frequency trend. Although higher (size-adjusted) power is typically achieved using $\ell=4$ in Panel A (compared with Panel B), such a low maximum augmentation would not, in general, be recommended due to potential size distortions for more general disturbance processes. It is also notable that the use of the OLS-GLS procedure has relatively little impact on power relative to GLS detrending for the low orders in Panel A, but increases power in Panel B especially for the single parameter statistics $t_{0}$ and $t_{2}$ with $M A I C$ lag selection and for alternatives further from the null hypothesis. However, power is already high for the joint seasonal unit root tests $F_{12}$ and $F_{012}$ with $c=10$ or 20 in both panels of the table and for these the $P Q$ variant has little effect.

Many of the patterns revealed in Table 4 carry over to the more general processes of Tables 5 and 6. In particular, GLS detrending typically leads to power gains over OLS for near-integrated processes, which in Tables 5 and 6 are seen particularly in the joint test statistics $F_{1}, F_{12}$ and $F_{012}$ and apply irrespective of whether lag selection is undertaken through an information criterion or testing approach. The power gains for $t_{2}$ from GLS detrending are also substantial when $c=5,10$, except in Panels A and B of Table 5. The sometimes substantial power loss exhibited by GLS detrending in these latter cases occurs when the root of the MA disturbance process at the Nyquist frequency is close to cancelling with the corresponding AR root and tend to be more severe as distance from the unit root null increases. However, this power loss is mitigated in the case of MAIC when OLS-GLS detrending is employed, and this is investigated further in the following subsection. Across both Tables 5 and 6 , and in line with the white noise processes of Table 4, relatively modest (if any) size-adjusted power gains over OLS typically apply for the zero frequency statistic $t_{0}$ with GLS detrending, apparently due to the inclusion of a zero frequency trend component. Away from the unit root null (represented by $c=20$ ), the value of OLS-GLS over GLS detrending with $M A I C$ or $S M A I C$ lag selection is seen for the seasonal MA process with $\Phi=0.5$ (Panel B of Table 5), but little or no power gains are generally evident when no near-cancellation applies across (3.1), namely in Panel C of Table 5 and both panels of Table 6.

Although the use of a $5 \%$ (rather than 10\%) significance level for lag selection based on the $t$-sq testing approach improves the power of tests a little, parsiminony does not always improve sizeadjusted power for information criteria lag specification methods. For example, seasonal unit root tests with $A I C$ lag specification have higher power than those using $B I C$ in Panel A of Table 6 when $c=20$. Nevertheless, the use of $M A I C$ or $M B I C$ almost always leads to tests with lower size-adjusted power than $A I C$ or $B I C$ (as appropriate); indeed these modified criteria lead to some results where power is less than size (see particularly Panel A of Table 6). The sequential approaches

\footnotetext{
${ }^{7}$ The results for other cases are available on request.
} 
are designed to improve power by eliminating redundant interediate lags, and this is clearly occurs for $t$-bm and $t$-rt compared with $t$-sq. On the other hand, however, $S A I C$ is not reliable in yielding power improvements relative to $A I C$. Perron and $\mathrm{Qu}(2007)$ discuss the problem that $M A I C$ can be prone to power reversal as $c$ increases, and this is seen for size-adjusted power for both $M A I C$ and $S M A I C$ for the positively autocorrelated seasonal AR process of Table 6 Panel A and also for SMAIC in Table 5 Panel C (positively autocorrelated seasonal MA). Power reversal issues are considered further in the next subsection.

The results of Appendix Tables A.4 to A.6, which correspond to Tables 4 to 6 but employ a sample size of $N=100$ years, overall exhibit similar patterns to those just discussed. This is the case because we use local-to-unity DGPs, so that power is largely constant for $N=60,100$. However, the power reversal problem for $M A I C$ and $S M A I C$ does not occur for positively autocorrelated seasonal processes with the larger sample size in Appendix Tables A.5 (Panel C) and A.6 (Panel A). It is also noteworthy that there remain cases in Panel A of Table A.6 where MBIC leads to tests with power less than size, with this occuring for the zero frequency test with all detrending methods ${ }^{8}$

\subsection{MAIC Power Functions}

The results in the tables confirm that the size and power of seasonal unit root tests depend on the treatment of the deterministic component, in addition to the lag selection method employed, and that relative performance can change with distance from the unit root null hypothesis. Therefore, the present subsection further examines the implications of the treatment of $\mu_{4 t+s}$ for the zero, Nyquist and annual frequency tests in Figures 1, 2 and 3, respectively. In particular, we investigate whether the OLS-GLS detrending method of Perron and $\mathrm{Qu}$ (2007) yields improved power performance relative to GLS detrending for DGPs away from the unit root null. Those authors are concerned with zero frequency tests and employ $M A I C$ lag selection (developed by $\mathrm{Ng}$ and Perron 2001), while the seasonal unit root extension here employs the seasonal $M A I C$ of subsection 2.3 above. As in the tables, the regressions have $k_{\max }=\left\lfloor 12(4 N / 100)^{1 / 4}\right\rfloor$. Using an analogous notation to Perron and Qu (2007), lag specification with deterministics included in the HEGY regression (2.5) is denoted in the figures as ols_ols, while GLS detrending as proposed by Rodrigues and Taylor (2007) for the seasonal unit root case is denoted as gls_gls. Finally, the hybrid version of Perron and Qu (2007) is denoted in the graphs as ols_gls.

The DGP again takes the form (3.1). To be specific, we employ 51 values of $c$ such that $c / N=$ $0,0.02 N, 0.04 N, \ldots, 1$, hence moving progressively from an integrated process with seasonal AR coefficient in (3.1) of $\alpha_{4}=(1-c / N)=1$ to a process with $\alpha_{4}=0 ; 50,000$ replications are used for each $c$. White noise innovations with $u_{4 t+s}=\varepsilon_{4 t+s}$ in $(3.2$ are employed in panels (a) and (b) of each figure, while the seasonal MA of panels (c) and (d) specifies $\Theta=0.5$ in 3.2 ; note again that the latter case has moving average roots relatively close to the AR seasonal unit roots under the null

\footnotetext{
${ }^{8}$ For both $N=60$ and $N=100$, the corresponding results with $M B I C$ lag selection and GLS detrending exhibit similar patterns to those shown with OLS-GLS detrending.
} 
hypothesis, and hence represents the situation for which $M A I C$ is designed ( $\mathrm{Ng}$ and Perron, 2001). A direct comparison is made of the effect of $N$ on size and power by showing results for $N=60$ in panels (a) and (c), with corresponding size results for $N=100$ in panels (b) and (d). To facilitate comparison with the results of Perron and Qu (2007), our figures show empirical size $(c / N=0)$ and power $(c / N>0)$ for tests at a $5 \%$ nominal significance level throughout. Consequently, power here cannot be directly compared with size-adjusted power presented in Table 4 (Panel B) and 5 (Panel B), and the corresponding appendix tables. Nevertheless, as for the tables, the test regression allows for seasonal intercepts and a zero frequency trend.

The distinctive power implications of the detrending methods are evident for the zero frequency test and white noise disturbances $(N=60)$ in panel (a) of Figure 1. Thus, GLS detrending yields substantial power advantages over OLS for near-integrated processes, but the latter has greater power for processes further from the unit root null. Indeed, with GLS detrending power flattens off at around 0.7 for a seasonal AR coefficient of $\alpha_{4}$ equal to about 0.65 or less in (3.2). The best method is hybrid OLS-GLS detrending, which here combines the advantages of both of the other methods. Power is, of course, higher for a given seasonal AR coefficient for the larger sample size of $N=100$ in panel (b) compared with panel (a) and otherwise the general patterns just discussed apply also in panel (b). Nevertheless, the flattening of power with GLS detrending now occurs for a value of $\alpha_{4}$ around 0.75 $(c \geq 25)$ and some evidence of power reversal, as documented in the figures of Perron and Qu (2007) for this method, can be seen for processes far from the unit root null.

As already discussed in relation to Panel B of Table 2, panel (c) of Figure 1 shows the zero frequency unit root tests to be oversized when the DGP has a seasonal MA disturbance with $\Theta=0.5$. As also seen in Panel B of Table 5, GLS detrending provides little or no power advantage over OLS for small values of $c$ in this near-cancellation context. However, OLS-GLS detrending results in greater oversizing than other methods and nominally greater power to about $\alpha_{4}=0.67(c=20)$; thereafter OLS and OLS-GLS have effectively the same power, which is substantially above that obtained with GLS detrending. For $N=100$ in panel (d), power reversal again occurs with GLS detrending as $c$ increases. While not evident with the smaller sample size in panel (c), panel (d) shows an intermediate range of values of $c$ for which OLS detrending leads to power higher than that given by the OLS-GLS approach.

Although the $t_{0}$ and $t_{2}$ unit root test statistics have the same asymptotic distributions when corresponding deterministic components are included, our test regressions allow for a trend in relation to the former, but only an intercept for the latter. Therefore, Figure 2 relating to the test at frequency $\pi$ (the Nyquist frequency) is analogous to a nonseasonal unit root test with constant only. For white noise innovations, the power advantages of GLS and OLS-GLS detrending over OLS are very clear for processes close to the unit root null in both panels (a) and (b). It is also notable that, compared with the corresponding panels of Figure 1, GLS detrending shows clearer evidence of power reversal with increasing $c$ when no trend is allowed at the corresponding frequency. The patterns for the seasonal MA in panels (c) and (d) are broadly similar to those of the corresponding panels of Figure 1. However, it may be noted that power for processes very far from the null hypothesis is worse with 
GLS detrending when $N=100$ in panel (d) compared with $N=60$ in panel (c). Indeed, in the former case, power is only around 0.65 with when the seasonal AR coefficient in (3.1) is zero, whereas it is approximately 0.75 for the smaller sample size.

The power properties of the joint $F_{1}$ statistic for testing seasonal unit roots at the annual frequency in Figure 3 are generally similar to those for $t_{2}$ statistic at the Nyquist frequency (Figure 2). In particular, panels (a) and (b) again reveal evidence of power reversal when GLS detrending is employed with white noise innovations. There is also evidence in these panels that OLS detrending leads to a statistic with slightly more power than GLS or OLS-GLS when the true AR parameter in (3.1) is small, with values of around $\alpha_{4}=0.5$ or less (corresponding to $c$ greater than 30 or 50 for $N=60$ or 100, respectively). Perhaps most remarkable is the low power attained by the joint test statistic with GLS detrending in panels (c) and (d) of Figure 3 against alternatives distant from the null hypothesis. Specifically, when the disturbances of (3.1) follow the seasonal MA of 3.2 with $\Theta=0.5$, the maximum power attained is only around 0.65 when $N=60$. While maximum power is a little higher for the larger sample size of $N=100$ years, power reversal as alternatives further from the null are considered implies that it is only around 0.55 for a process with $\alpha_{4}=0$. Panels (c) and (d) show a fairly wide range of parameters for which OLS detrending yields tests with higher power than either version of GLS detrending; this good performance of OLS detrending is also evident for size-adjusted power in Panel B of Tables 5 and A.5 with $c=20$. Nevertheless, and as anticipated, GLS detrending methods have substantially higher power than OLS for local-to-unity alternatives.

\subsection{Implications for Empirical Analysis}

The Monte Carlo results of the preceding subsections have important implications for empirical analysis. Although the size results across Tables 1-3, together with Appendix Tables A.1 to A.3, indicate that no single method always leads to good size, nevertheless our extension of $M A I C$ for seasonal unit root testing delivers relatively good size across a range of DGPs and irrespective of whether it is used in the HEGY regression (2.5) or after GLS detrending in 2.11). In common with $\mathrm{Ng}$ and Perron (2001), we do not recommend BIC-based procedures as they suffer more from size distortions. If dynamic specification is based on significance of lag coefficients, then $t-s q(10 \%)$ has the best size overall, although it is badly over-sized in the presence of near-cancellation with MA roots. Although it seems intuitively plausible that deletion of intermediate lags may improve lag specification for seasonal processes, we do not recommend the use of such procedures (either information criteria or testingbased) due to the poorly sized unit root tests that can result. It also needs to be emphasised that all procedures we examine in detail allow the same maximum lag of $\left\lfloor 12(4 N / 100)^{1 / 4}\right\rfloor$, equating to 14 quarters for $N=60$ years of data. Any procedure that starts from a low maximum lag of (say) 4 or 5 quarters can have poor size in the presence of MA disturbances (even of low order) or an AR process of order higher than $k_{\max }$.

The results for size-adjusted power in Tables 4 to 6 and Appendix Tables A.4 to A.6 need to be interpreted in the light of size considerations. The price in terms of power for the good size 
performance of $M A I C$ is that it can have fairly substantial power loss compared with $A I C$ for some DGPs (for example, Panels B and C of Table 5 and Panel A of Table 6). Although this power loss is often mitigated (or even reversed) by the use of the sequential $S M A I C$, the very poor size of the latter for some processes makes empirical results difficult to interpret in practice. Nevertheless, the low power of zero frequency unit root tests (which allow a trend) with $M A I C$ lag specification for the positively autocorrelated seasonal processes of Panel $\mathrm{C}$ of Table 5 and (especially) Panel A of Table 6 with $N=60$ years of data shows that this method does not perform universally well. Our results confirm that GLS detrending often has power advantages over OLS detrending for near-integrated processes, with this particularly evident in panels (a) and (b) of Figures 1 to 3. However, all figures show also that GLS detrending with lag specification undertaken in the context of the GLS regression has relatively poor power away from the unit root null hypothesis and is dominated by the OLS-GLS procedure of Perron and Qu (2007). Nevertheless, when near-cancellation applies across the AR and MA polynomials of (3.1), as in panels (c) and (d) in the figures and Panel B of Table 5, OLS detrending can have higher power than OLS-GLS.

Although there is some variation over the DGPs considered, the results of Tables 1 to 3 suggest that OLS detrending with $M A I C$ or $A I C$ may have more reliable size overall than testing in conjunction with GLS (or OLS-GLS detrending). On the other hand, power against local alternatives is typically higher with GLS-based detrending. The implied size-power trade-off indicates that test results with OLS detrending may also contain useful information, alongside those with GLS (especially OLS-GLS) detrending.

In summary, therefore, we recommend that our seasonal generalisation of $M A I C$ be routinely used for lag specification when undertaking seasonal unit root tests. In terms of detrending, the OLS-GLS procedure generally works well, and apparently avoids the low power that can result from seasonal GLS detrending for processes away from the unit root null. However, prior to unit root testing, it is advisable that empirical researchers explicitly consider the properties of the series they are examining, rather than applying seasonal unit root tests as a "black box" procedure. MAIC was designed by $\mathrm{Ng}$ and Perron (2001) to deal with over-rejection of the (zero frequency) unit root null hypothesis for an integrated process that is also subject to negatively autocorrelated disturbances. Our simulations show that the seasonal MAIC generalisation leads to tests with good size in the corresponding case of negatively autocorrelated innovations of a seasonal form, but also indicate that $A I C$ has quite good size when the process has positively autocorrelated innovations at seasonal lags (Panel $\mathrm{C}$ of Table 2 and Panel A of Table 3) and better size-adjusted power than $M A I C$ for corresponding near-integrated local alternatives (Panel $\mathrm{C}$ of Table 5 and Panel A of Table 6).

\section{Empirical Application}

To investigate the implications of different methodologies for lag selection and accounting for trends in the context of HEGY-type seasonal unit roots, the procedures investigated in the Monte Carlo 
analysis of Section 3 are applied to observed US quarterly industrial production indices (IPI). More specifically, we employ the logarithm of IPI for the component series of business equipment, business supplies, construction supplies, durable consumer goods, durable materials goods and non-durable consumer goods for the US over 1947Q1 to 2010Q49 in aggregate these constitute approximately 70 percent of US industrial production.

As evident from the graphs of the series in Figure 4, all IPI series exhibit trends and seasonality, with the effect of the recent recession also often evident (particularly for construction supplies). Further insight into the properties of these series is provided by Table 7, where (in the general notation of 2.1a) coefficient estimates are reported for parsiminous seasonal ARMA models of the general form ${ }^{10}$

$$
(1-\phi L)\left(1-\Phi L^{4}\right) \Delta x_{4 t+s}=\Delta \mu_{4 t+s}+\left(1-\theta_{1} L-\theta_{2} L^{2}\right)\left(1-\Theta L^{4}\right) \varepsilon_{4 t+s} .
$$

The need for a zero frequency unit root is relatively uncontroversial (see Table 8 below) and is imposed in 4.1. Although they are not always explicitly allowed in such models, our specification includes deterministic seasonal dummy variables in $\Delta \mu_{4 t+s}$, since the exclusion of deterministic seasonality results in all estimated $\widehat{\Phi}$ being close to unity.

Both Figure 4 and the results in Table 7 (specifically coefficient estimates and $R^{2}$ values) indicate that business equipment and durable goods materials have relatively modest seasonality. While construction supplies also exhibits relatively little stochastic seasonality in terms of $\widehat{\Phi}$, this IPI component shows strong deterministic seasonality in Figure 4. On the other hand, the ARMA models for business supplies, durable consumer goods and non-durable consumer goods suggest strong stochastic seasonality which may be of a nonstationary form. After imposition of a zero frequency unit root through first differencing, all IPI components show positive first-order serial correlation, which is usually captured through an MA(1).

Against this background, Table 8 shows results of HEGY tests applied to the log series (without prior differencing) using a range of lag selection procedures. To illustrate the impact of decisions made with respect to lag selection and detrending, we consider conventional $A I C$ and $B I C$ lag selection procedures, together our seasonal $M A I C$ and the corresponding sequential version $S M A I C$ that allows "gaps" in the lag structure. Modified criteria based on $B I C$ are not included, since they have worse size performance in the finite sample Monte Carlo analysis of Section 3 than the corresponding $A I C$-based procedures. Similarly $S A I C$ is not included, due to its size distortions. For comparison purposes, results are also shown for the testing-based lag selection procedures $t$-sq(10\%) and $t$-rt $(10 \%)$, the latter of which also allows some intermediate lags to be dropped from the test regression. In line with many empirical studies, the maximum lag order is set as $k_{\max }=\left\lfloor 12(4 N / 100)^{1 / 4}\right\rfloor$, which implies $k_{\max }=15$ for our sample of $N=64$ years of data. Both OLS and GLS detrending methods are used, with the Perron and Qu (2007) hybrid OLS-GLS method employed for the latter with $M A I C$ and $S M A I C$ lag selection, in line with the generally superior performance of OLS-GLS over

\footnotetext{
${ }^{9}$ All data are from the US Federal Reserve website http://www.federalreserve.gov/releases/g17/table1_2.htm.

${ }^{10}$ This general specification was adopted based on the serial correlation properties of the first differenced series. The ARMA models were estimated in the program EViews using conditional least squares.
} 
GLS detrending for these criteria in the Monte Carlo analysis of the previous section. To facilitate comparisons, Table 8 indicates the lags selected by each method.

As implied above, almost all results for $t_{0}$ in Table 8 are compatible with the presence of a zero frequency unit root, with ambiguity in this respect only for business equipment with $A I C$ or $B I C$ lag selection. Note, however, that conventional $A I C / B I C$ used in the context of HEGY tests that perform OLS detrending through (2.5) here sometimes result in low orders of augmentation being selected; indeed, in three cases (including business equipment) no lags are selected. These parsiminous models also yield numerically large values for the seasonal unit root test statistics. However, given the large oversizing of these parsiminous lag selection procedures in the presence of a positively autocorrelated seasonal MA component (in Panel B of Table 2), such rejections may be spurious. Although the less parsimonious methods of $M A I C$ or $t$-sq(10\%) select very similar lags for both OLS and GLS detrending in Table 8, nevertheless the seasonal unit null hypotheses are rejected more frequently using OLS than the corresponding GLS-detrended tests of (2.11). For example, using $F_{12}$, the joint null hypothesis of unit roots at the Nyquist and annual frequencies is strongly rejected for construction supplies with OLS detrending irrespective of the lag selection method employed, whereas this hypothesis is rejected with GLS detrending only when the parsimonious BIC criterion is used.

Based on size and power properties, our recommendation in subsection 3.5 is that $M A I C$ be widely employed with OLS and OLS-GLS detrending, but also that the empirical characteristics of the series should be considered explicitly. Using $M A I C$ in conjunction with OLS-GLS detrending and at the conventional $5 \%$ significance level, the results in Table 8 indicate that the IPI components of business supplies, construction supplies, durable consumer goods and non-durable consumer goods are compatible with the seasonal integration hypothesis. Nevertheless, this conclusion is surprising for construction supplies in relation to the estimated seasonal AR coefficient obtained for this component in Table 7 and is not confirmed when OLS detrending is employed. The remaining two components, business equipment and durable goods materials, are those for which seasonality is not very marked in Figure 4 and these also have small $\widehat{\Phi}$ in Table 7 . For business equipment, the $M A I C /$ GLS test results in Table 8 are marginal, but these combined with the information from Table 7 and the higher power that can be shown by the use of OLS detrending point to rejection of the overall seasonal integration null hypothesis. The conclusion for durable goods materials is straightforward, namely rejection of unit roots at both seasonal frequencies.

Overall, therefore, we conclude that modelling the IPI components of business supplies, durable consumer goods and non-durable consumer goods may proceed on the basis that these components are seasonally integrated, whereas the business equipment and durable goods materials are integrated only at the zero frequency. Although the case of construction supplies is more ambiguous and more detailed modelling is warranted to shed further light on the possible presence of seasonal unit roots, nevertheless the results here point to only a zero frequency unit root being present. In any case, irrespective of the particular conclusions drawn in relation to these series, our results in Table 8 illustrate the important roles played by the methodogies used for lag selection and detrending when conducting seasonal unit root tests. 


\section{Conclusions}

Through Monte Carlo simulation experiments, this paper explores the small sample performance of a variety of data-based methods for determining the lag augmentation polynomial used for conducting HEGY seasonal unit root tests, in both the common OLS detrending context originally proposed by Hylleberg et al. (1990) and also employing seasonal GLS detrending as proposed by Rodrigues and Taylor (2007). A battery of techniques are compared for lag selection in these contexts, based on both information criteria $(A I C$ and $B I C)$ and also hypothesis testing approaches. One contribution of our paper is that we extend the modified information criteria of $\mathrm{Ng}$ and Perron (2001) to the seasonal unit root testing context and apply this for lag selection with both OLS and GLS detrending, in the latter case implemented for modified information criteria methods using both the GLS regression and the hybrid OLS-GLS approach recommended by Perron and Qu (2007). Although Taylor (1997) drew attention to the difficulty of lag selection for seasonal unit root tests, no comprehensive examination of the performance of parametric seasonal unit root tests has previously been available.

Our results imply that, over a range of data generating processes, reliable size is generally delivered by our seasonal generalisation of the modified Akaike criterion, $M A I C$, whether applied with OLS or GLS detrending. It is well known that the use of conventional lag selection criteria, such as $A I C$ or $B I C$, together with hypothesis testing approaches, can lead to badly over-sized unit root tests for processes with negatively autocorrelated moving average disturbances (Schwert, 1989, $\mathrm{Ng}$ and Perron, 2001), with an analogous problem applying to seasonal unit root tests in the presence of nearcancellation of roots of the AR and MA polynomials (Ghysels et al., 1994). We also find this result, but show that $M A I C$ performs well in delivering reliable size across a range of data generating processes. A sequential version $(S M A I C)$ also sometimes delivers good size, but unfortunately can be badly under-sized. Nevertheless, $M A I C$ can have poor size when the innovations exhibit positive seasonal autocorrelation, in which case $A I C$ is preferred. When used in appropriate contexts, MAIC/AIC deliver more reliable size than lag specification methods based on hypothesis testing or BIC.

In terms of power, the hybrid OLS-GLS detrending approach of Perron and Qu (2007) should be employed with $M A I C$ to avoid poor power shown by GLS detrending under alternatives distant from the unit root null. At least for local alternatives, GLS detrending is more powerful than OLS, but there may be parameter ranges over which OLS detrending has greater power than GLS-based methods.

An application of parametric HEGY-type seasonal unit root tests with OLS and GLS detrending and a range of lag selection procedures to six components of quarterly seasonally unadjusted US industrial production indices illustrates the different results that can be delivered in an empirical context. Although there is little disagreement about the presence of a zero frequency unit root, results with OLS detrending more frequently reject the presence of seasonal unit roots at the Nyquist (semiannual) and annual frequencies than with GLS. Use of $M A I C$ leads to similar conclusions for both OLS and GLS detrending, especially in relation to the joint test of the overall seasonal integration null hypothesis. 
To date, GLS (and, more especially, OLS-GLS) detrending does not appear to be widely used in empirical studies that test for seasonal unit roots. However, its performance in the Monte Carlo analysis and the different results it sometimes delivers, compared with the commonly applied OLS detrending, for US industrial production indices indicate that it may shed new light on the properties of observed seasonal time series.

\section{References}

Beaulieu, J.J. and Miron, J.A. (1993) Seasonal unit roots in aggregate U.S. data. Journal of Econometrics 55, 305-328.

Burridge, P. and Wallis, K.F. (1984). Unobserved-component models for seasonal adjustment filters. Journal of Business and Economic Statistics 2, 350-359.

Chang, Y. and Park, J.Y. (2002). On the asymptotics of ADF tests for unit roots. Econometric Reviews 21, 431-447.

Cleveland, W.P. and Tiao, G.C. (1976). Decomposition of seasonal time series: A model for the Census X-11 program. Journal of the American Statistical Association 71, 581-587.

del Barrio Castro T. and Osborn D.R. (2011). HEGY tests in the presence of moving averages. Oxford Bulletin of Economic and Statistics 73, 691-704.

del Barrio Castro T. and Osborn D.R. (2012). Non-parametric testing for seasonally and periodically integrated processes. Journal of Time Series Analysis 33, 424-437.

del Barrio Castro T., Osborn D.R. and Taylor, A.M.R. (2012). On augmented HEGY tests for seasonal unit roots. Econometric Theory 28, 1121-1143.

Elliott, G., Rothenberg, T.J. and Stock, J.H. (1996). Efficient tests for an autoregressive unit root. Econometrica 64, 813-836.

Ghysels, E., Lee, H. S., and Noh, J. (1994). Testing for unit roots in seasonal time series: some theoretical extensions and a Monte Carlo investigation. Journal of Econometrics 62, 415- 442.

Gregoir, S. (2006). Efficient tests for the presence of a pair of complex conjugate unit roots in real time series. Journal of Econometrics 130, 45-100.

Hall, A.R. (1994). Testing for a unit root in time series with pretest data-based model selection. Journal of Business and Economic Statistics 12, 461-70.

Hylleberg, S., Engle, R. F., Granger, C. W. J., and Yoo, B. S. (1990). Seasonal integration and cointegration. Journal of Econometrics 44, 215-238. 
Ng, S., and Perron, P. (1995). Unit root tests in ARMA models with data dependent methods for selection of the truncation lag. Journal of the American Statistical Association 90, 268-281.

Ng, S., and Perron, P. (2001). Lag length selection and the construction of unit root tests with good size and power. Econometrica 69, 1519-1554.

Perron, P., and Qu, Z. (2007). A simple modification to improve the finite sample properties of $\mathrm{Ng}$ and Perron's unit root tests. Economics Letters 94, 12-19.

Rodrigues, P. M. M., and Taylor A. M. R. (2004). Alternative estimators and unit root tests for seasonal autoregressive processes. Journal of Econometrics 120, 35-73.

Rodrigues, P. M. M., and Taylor A. M. R. (2007). Efficient tests of the seasonal unit root hypothesis. Journal of Econometrics 141, 548-573.

Schwert, G. (1989). Testing for unit roots: a Monte Carlo investigation. Journal of Business and Economic Statistics 7, 147-158.

Smith R.J., and Taylor, A.M.R. (1998). Additional critical values and asymptotic representations for seasonal unit root tests. Journal of Econometrics 85, 269-288.

Smith, R.J., and Taylor, A.M.R. (1999). Likelihood ratio tests for seasonal unit roots. Journal of Time Series Analysis 20, 453-476.

Smith R.J., Taylor, A.M.R., and del Barrio Castro, T. (2009). Regression-based seasonal unit root tests. Econometric Theory 25, 527-560.

Taylor, A. M. R. (1997). On the practical problems of computing seasonal unit root tests. International Journal of Forecasting 13, 307-318.

Taylor, A. M. R. (2005). Variance ratio tests of the seasonal unit root hypothesis. Journal of Econometrics 124, 33-54. 
Table 1: Empirical size of quarterly seasonal unit root tests for white noise disturbances

\begin{tabular}{|c|c|c|c|c|c|c|c|c|c|c|}
\hline & \multicolumn{2}{|c|}{$t_{0}$} & \multicolumn{2}{|c|}{$t_{2}$} & \multicolumn{2}{|c|}{$F_{1}$} & \multicolumn{2}{|c|}{$F_{12}$} & \multicolumn{2}{|c|}{$F_{012}$} \\
\hline & $O L S$ & $G L S$ & $O L S$ & $G L S$ & $O L S$ & $G L S$ & $O L S$ & $G L S$ & $O L S$ & $G L S$ \\
\hline \multicolumn{11}{|l|}{ Panel A. $\ell=4$} \\
\hline$A I C$ & 51 & 0.071 & 047 & 0.082 & 0.055 & 0.063 & 0.052 & 0.072 & 0.055 & 0.089 \\
\hline$M A I C$ & 0.039 & 0.053 & 0.040 & 0.075 & 0.047 & 0.052 & 0.042 & 0.063 & 0.042 & 0.070 \\
\hline$M A I C_{-} P Q$ & & 0.053 & & 0.075 & & 0.053 & & 0.064 & & 0.071 \\
\hline$B I C$ & 0.050 & 0.069 & 0.045 & 0.081 & 0.054 & 0.061 & 0.049 & 0.071 & 0.052 & 0.084 \\
\hline$M B I C$ & 0.040 & 0.057 & 0.042 & 0.078 & 0.049 & 0.055 & 0.044 & 0.064 & 0.044 & 0.073 \\
\hline$M B I C \_P Q$ & & 0.057 & & 0.077 & & 0.055 & & 0.065 & & 0.074 \\
\hline$S A I C$ & 056 & 0.072 & 048 & 0.081 & 0.053 & 0.064 & .056 & 0.077 & .060 & 0.096 \\
\hline$S M A I C$ & 0.025 & 0.045 & 0.036 & 0.069 & 0.039 & 0.050 & 0.035 & 0.060 & 0.033 & 0.066 \\
\hline$S M A I C \_P Q$ & & 0.045 & & 0.069 & & 0.050 & & 0.060 & & 0.067 \\
\hline$t-s q(5 \%)$ & 0.055 & 0.072 & 047 & 0.083 & 0.055 & 0.063 & 0.052 & 0.073 & 0.059 & 0.092 \\
\hline$t-s q(10 \%)$ & 0.056 & 0.075 & 0.048 & 0.083 & 0.056 & 0.063 & 0.052 & 0.075 & 0.060 & 0.095 \\
\hline$t-b m(5 \%)$ & 0.056 & 0.070 & 0.047 & 0.079 & 0.056 & 0.068 & 0.054 & 0.080 & 0.059 & 0.100 \\
\hline$t-b m(10 \%)$ & 0.058 & 0.073 & 0.049 & 0.081 & 0.057 & 0.070 & 0.055 & 0.081 & 0.062 & 0.104 \\
\hline$t-r t(5 \%)$ & 0.053 & 0.070 & 0.047 & 0.080 & 0.053 & 0.064 & 0.055 & 0.076 & 0.058 & 0.093 \\
\hline$t-r t(10 \%)$ & 0.056 & 0.073 & 0.048 & 0.082 & 0.053 & 0.064 & 0.056 & 0.078 & 0.061 & 0.096 \\
\hline \multicolumn{11}{|l|}{ Panel B. $\ell=12$} \\
\hline$A I C$ & 0.054 & 0.068 & 0.051 & 0.078 & 0.054 & 0.060 & 0.050 & 0.069 & 0.055 & 0.084 \\
\hline$M A I C$ & 0.039 & 0.048 & 0.044 & 0.067 & 0.044 & 0.048 & 0.041 & 0.055 & 0.041 & 0.062 \\
\hline$M A I C \_P Q$ & & 0.049 & & 0.070 & & 0.050 & & 0.057 & & 0.064 \\
\hline$B I C$ & $\overline{051}$ & 0.065 & 0.051 & 0.077 & 0.052 & 0.058 & 0.048 & 0.066 & 0.050 & 0.079 \\
\hline$M B I C$ & 0.042 & 0.052 & 0.046 & 0.074 & 0.047 & 0.054 & 0.043 & 0.060 & 0.043 & 0.068 \\
\hline$M B I C \_P Q$ & & 0.054 & & 0.075 & & 0.053 & & 0.060 & & 0.069 \\
\hline$S A I C$ & .082 & 0.087 & 0.064 & 0.091 & 0.070 & 0.071 & 0.070 & 0.085 & 0.085 & 0.114 \\
\hline$S M A I C$ & 0.008 & 0.022 & 0.021 & 0.049 & 0.017 & 0.031 & 0.015 & 0.037 & 0.010 & 0.033 \\
\hline$S M A I C \_P Q$ & & 0.023 & & 0.048 & & 0.031 & & 0.037 & & 0.036 \\
\hline$t-s q(5 \%)$ & 0.069 & 0.082 & 0.057 & 0.083 & 0.059 & 0.064 & 0.055 & 0.075 & 0.067 & 0.096 \\
\hline$t-s q(10 \%)$ & 0.070 & 0.081 & 0.056 & 0.086 & 0.053 & 0.061 & 0.051 & 0.077 & 0.065 & 0.094 \\
\hline$t-b m(5 \%)$ & 0.075 & 0.081 & 0.061 & 0.083 & 0.063 & 0.073 & 0.061 & 0.086 & 0.074 & 0.114 \\
\hline$t-b m(10 \%)$ & 0.088 & 0.096 & 0.067 & 0.088 & 0.070 & 0.075 & 0.069 & 0.093 & 0.089 & 0.130 \\
\hline$t-r t(5 \%)$ & 0.076 & 0.079 & 0.058 & 0.085 & 0.067 & 0.069 & 0.063 & 0.081 & 0.073 & 0.103 \\
\hline$t-r t(10 \%)$ & 0.085 & 0.088 & 0.064 & 0.092 & 0.071 & 0.072 & 0.072 & 0.086 & 0.086 & 0.116 \\
\hline
\end{tabular}

Notes: The DGP is (3.1) with $\mathrm{c}=0$ and $u_{4 t+s}=\varepsilon_{4 t+s} \sim I I D N(0,1)$, for quarterly data over $N=60$ years. Tests and lag selection criteria as in Section 2 , with $k_{\max }=\operatorname{int}[\ell(4 \mathrm{~N} / 100)]^{1 / 4}$ for $\ell=4$ or 12 . All tests allow for seasonal means and a zero frequency trend: $O L S$ and GLS indicates OLS-detrending and GLS-detrending, with PQ indicating that the latter uses the OLS-GLS method of Perron and Qu (2007). The statistics are $t$-type tests for unit roots at the zero and $\pi$ frequencies $\left(t_{0}, t_{2}\right)$ and joint $F$-type statistics for unit roots at the $\pi / 2$ frequency. $\left(F_{1}\right)$, all seasonal frequencies $\left(F_{12}\right)$ and the zero and all seasonal frequencies $\left(F_{012}\right)$. Results are based on 5000 replications for a nominal $5 \%$ level of significance. 
Table 2: Empirical size of quarterly seasonal unit root tests for moving average disturbances

\begin{tabular}{|c|c|c|c|c|c|c|c|c|c|c|}
\hline & \multicolumn{2}{|c|}{$t_{0}$} & \multicolumn{2}{|c|}{$t_{2}$} & \multicolumn{2}{|c|}{$F_{1}$} & \multicolumn{2}{|c|}{$F_{12}$} & \multicolumn{2}{|c|}{$F_{012}$} \\
\hline & $O L S$ & $G L S$ & $O L S$ & $G L S$ & $O L S$ & $G L S$ & $O L S$ & $G L S$ & $O L S$ & $G L S$ \\
\hline \multicolumn{11}{|c|}{ Panel A. $M A(1): \theta=-0.8, \Theta=0$} \\
\hline$A I C$ & 0.057 & 0.080 & 0.301 & 0.322 & 0.059 & 0.069 & 0.247 & 0.238 & 0.241 & 0.235 \\
\hline$M A I C$ & 0.024 & 0.031 & 0.072 & 0.132 & 0.036 & 0.039 & 0.066 & 0.072 & 0.059 & 0.066 \\
\hline$M A I C \quad P Q$ & & 0.035 & & 0.137 & & 0.043 & & 0.084 & & 0.077 \\
\hline$B I C$ & 0.064 & 0.082 & 0.497 & 0.500 & 0.065 & 0.076 & 0.420 & 0.397 & 0.402 & 0.376 \\
\hline$M B I C$ & 0.022 & 0.025 & 0.092 & 0.185 & 0.032 & 0.035 & 0.074 & 0.091 & 0.066 & 0.074 \\
\hline$M B I C_{-} P Q$ & & 0.031 & & 0.180 & & 0.041 & & 0.099 & & 0.086 \\
\hline$S A I C$ & 0.080 & 0.095 & 0.249 & 0.264 & 0.071 & 0.075 & 0.219 & 0.206 & 0.226 & 0.215 \\
\hline$S M A I C$ & 0.005 & 0.018 & 0.048 & 0.126 & 0.018 & 0.033 & 0.039 & 0.070 & 0.023 & 0.052 \\
\hline$S M A I C \quad P Q$ & & 0.018 & & 0.121 & & 0.035 & & 0.073 & & 0.055 \\
\hline$t-s q(5 \%)$ & 0.065 & 0.083 & 0.197 & 0.240 & 0.056 & 0.070 & 0.173 & 0.177 & 0.175 & 0.187 \\
\hline$t-s q(10 \%)$ & 0.059 & 0.081 & 0.128 & 0.167 & 0.057 & 0.066 & 0.121 & 0.126 & 0.121 & 0.140 \\
\hline$t-b m(5 \%)$ & 0.063 & 0.074 & 0.277 & 0.276 & 0.061 & 0.072 & 0.225 & 0.214 & 0.217 & 0.214 \\
\hline$t-b m(10 \%)$ & 0.065 & 0.075 & 0.223 & 0.230 & 0.059 & 0.070 & 0.194 & 0.184 & 0.189 & 0.185 \\
\hline$t-r t(5 \%)$ & 0.078 & 0.091 & 0.308 & 0.315 & 0.072 & 0.075 & 0.268 & 0.246 & 0.267 & 0.253 \\
\hline$t-r t(10 \%)$ & 0.079 & 0.094 & 0.240 & 0.257 & 0.072 & 0.075 & 0.211 & 0.200 & 0.219 & 0.213 \\
\hline \multicolumn{11}{|c|}{ Panel B. Seasonal $M A: \theta=0, \Theta=0.5$} \\
\hline$A I C$ & 0.267 & 0.260 & 0.192 & 0.233 & 0.209 & 0.140 & 0.265 & 0.228 & 0.363 & 0.348 \\
\hline$M A I C$ & 0.079 & 0.091 & 0.072 & 0.128 & 0.081 & 0.057 & 0.098 & 0.089 & 0.126 & 0.119 \\
\hline$M A I C_{-} P Q$ & & 0.112 & & 0.136 & & 0.067 & & 0.106 & & 0.144 \\
\hline$B I C$ & 0.544 & 0.478 & 0.419 & 0.395 & 0.486 & 0.291 & 0.584 & 0.429 & 0.672 & 0.562 \\
\hline$M B I C$ & 0.097 & 0.121 & 0.084 & 0.157 & 0.091 & 0.068 & 0.113 & 0.113 & 0.151 & 0.157 \\
\hline$M B I C_{-} P Q$ & & 0.130 & & 0.158 & & 0.075 & & 0.123 & & 0.175 \\
\hline$S A I C$ & 0.203 & 0.174 & 0.177 & 0.192 & 0.180 & 0.119 & 0.214 & 0.171 & 0.234 & 0.216 \\
\hline$S M A I C$ & 0.024 & 0.034 & 0.074 & 0.115 & 0.077 & 0.071 & 0.074 & 0.086 & 0.037 & 0.057 \\
\hline$S M A I C \_P Q$ & & 0.035 & & 0.116 & & 0.071 & & 0.086 & & 0.063 \\
\hline$t-s q(5 \%)$ & 0.158 & 0.172 & 0.113 & 0.165 & 0.125 & 0.093 & 0.154 & 0.146 & 0.216 & 0.218 \\
\hline$t-s q(10 \%)$ & 0.128 & 0.146 & 0.092 & 0.142 & 0.100 & 0.077 & 0.116 & 0.118 & 0.167 & 0.178 \\
\hline$t-b m(5 \%)$ & 0.214 & 0.178 & 0.192 & 0.210 & 0.199 & 0.143 & 0.246 & 0.206 & 0.264 & 0.257 \\
\hline$t-b m(10 \%)$ & 0.216 & 0.181 & 0.171 & 0.195 & 0.176 & 0.129 & 0.217 & 0.185 & 0.244 & 0.236 \\
\hline$t-r t(5 \%)$ & 0.201 & 0.172 & 0.186 & 0.201 & 0.196 & 0.133 & 0.233 & 0.188 & 0.250 & 0.226 \\
\hline$t-r t(10 \%)$ & 0.204 & 0.173 & 0.173 & 0.191 & 0.175 & 0.117 & 0.208 & 0.169 & 0.233 & 0.213 \\
\hline
\end{tabular}


Table 2 (continued)

\begin{tabular}{|c|c|c|c|c|c|c|c|c|c|c|}
\hline & \multicolumn{2}{|c|}{$t_{0}$} & \multicolumn{2}{|c|}{$t_{2}$} & \multicolumn{2}{|c|}{$F_{1}$} & \multicolumn{2}{|c|}{$F_{12}$} & \multicolumn{2}{|c|}{$F_{012}$} \\
\hline & $O L S$ & $G L S$ & $O L S$ & $G L S$ & $O L S$ & $G L S$ & $L S$ & $G L$ & $O L S$ & $G L S$ \\
\hline \multicolumn{11}{|c|}{ nal $M A: \theta=0, \Theta=-0.5$} \\
\hline$A I C$ & 0.083 & 0.111 & 0.067 & 0. & 0.076 & 0.011 & 083 & ט. & .106 & 0.126 \\
\hline$M A I C$ & 25 & 0.034 & 7 & 0.0 & 0 & 0.040 & 13 & 0.042 & .033 & 0.042 \\
\hline$M A$ & & 0.034 & & 0.0 & & 0.042 & & 0.1 & & 044 \\
\hline$B I C$ & 98 & 0.131 & 078 & 0.135 & 084 & 0.075 & 097 & 0.111 & 126 & 0.156 \\
\hline$M B I C$ & 0 & 0.012 & 0.0 & 0.031 & 0.034 & 0.035 & 0.028 & 0.031 & 0.016 & 0.022 \\
\hline$M B I C \_P Q$ & & 0.006 & & 0.020 & & 0.054 & & 0.045 & & 0.023 \\
\hline & 93 & 0.111 & & 0.1 & U४5 & 0.0 & 092 & 0.099 & 103 & 0.141 \\
\hline$I C$ & 002 & 0.014 & 0.019 & 0.048 & 0.019 & 0.030 & 0.015 & 0.029 & 0.009 & 0.022 \\
\hline$S M A I C_{-} P Q$ & & 0.013 & & 0.047 & & 0.031 & & 0.030 & & 0.024 \\
\hline$t_{-} s a b$ & 63 & 0.080 & & 0.0 & 5 & 0.0 & 069 & 0.072 & .077 & 0.094 \\
\hline$t-s q(1$ & 061 & 0.081 & 0.054 & 0.084 & 0.061 & 0.059 & 0.063 & 0.070 & 0.069 & 0.093 \\
\hline$t-b m(5 \%)$ & 0.077 & 0.099 & 0.064 & 0.100 & 0.079 & 0.080 & 0.083 & 0.098 & 0.092 & 0.131 \\
\hline$t-b m(10 \%$ & .083 & 0.108 & 0.066 & 0.101 & 0.081 & 0.081 & 0.087 & 0.100 & 0.097 & 0.142 \\
\hline & & 0.1 & 064 & & 83 & & 088 & & 0.098 & 0.134 \\
\hline$t-r t(10 \%)$ & 0.095 & 0.112 & 0.068 & 0.107 & 0.084 & 0.080 & 0.092 & 0.099 & 0.102 & 0.143 \\
\hline
\end{tabular}

Notes: As for Table 1, except that the DGP has moving average disturbances, with $u_{4 t+s}=(1-\theta L)\left(1-\Theta L^{4}\right) \varepsilon_{4 t+s}$ and maximum lag given by $k_{\max }=i n t\left[\ell(4 N / 100)^{1 / 4}\right]$ with $\ell=12$. 
Table 3: Empirical size of quarterly seasonal unit root tests for autoregressive disturbances

\begin{tabular}{|c|c|c|c|c|c|c|c|c|c|c|}
\hline & \multicolumn{2}{|c|}{$t_{0}$} & \multicolumn{2}{|c|}{$t_{2}$} & \multicolumn{2}{|c|}{$F_{1}$} & \multicolumn{2}{|c|}{$F_{12}$} & \multicolumn{2}{|c|}{$F_{012}$} \\
\hline & $O L S$ & $G L S$ & $O L S$ & $G L S$ & $O L S$ & $G L S$ & $O L S$ & $G L S$ & $O L S$ & $G L S$ \\
\hline \multicolumn{11}{|c|}{ Panel A. Seasonal $A R: \Phi=0.5$} \\
\hline$A I C$ & 0.047 & 0.070 & 0.048 & 0.086 & 0.053 & 0.064 & 0.059 & 0.075 & 0.060 & 0.087 \\
\hline$M A I C$ & 0.033 & 0.048 & 0.042 & 0.075 & 0.047 & 0.051 & 0.048 & 0.058 & 0.047 & 0.061 \\
\hline$M A I C_{-} P Q$ & & 0.048 & & 0.076 & & 0.052 & & 0.060 & & 0.063 \\
\hline$B I C$ & 042 & 0.066 & 0.047 & 0.084 & 0.054 & 0.063 & 0.058 & 0.073 & 0.058 & 0.083 \\
\hline$M B I C$ & 0.017 & 0.031 & 0.031 & 0.060 & 0.040 & 0.046 & 0.036 & 0.047 & 0.028 & 0.041 \\
\hline$M B I C_{-} P Q$ & & 0.020 & & 0.041 & & 0.057 & & 0.051 & & 0.035 \\
\hline$S A I C$ & 885 & 0.110 & 065 & 0.102 & 080 & 0.086 & 084 & 0.105 & .101 & 0.141 \\
\hline$S M A I C$ & 0.005 & 0.024 & 0.018 & 0.058 & 0.019 & 0.033 & 0.019 & 0.038 & 0.012 & 0.033 \\
\hline$S M A I C \_P Q$ & & 0.025 & & 0.058 & & 0.036 & & 0.039 & & 0.033 \\
\hline$t-s q(5 \%)$ & 0.055 & 0.081 & 0.050 & 0.085 & 0.058 & 0.069 & 0.062 & 0.081 & 0.067 & 0.098 \\
\hline$t-s q(10 \%)$ & 0.057 & 0.083 & 0.051 & 0.086 & 0.057 & 0.071 & .062 & 0.081 & 0.069 & 0.099 \\
\hline$t-b m(5 \%)$ & 0.067 & 0.095 & 0.055 & 0.095 & 0.067 & 0.078 & 0.071 & 0.095 & 0.082 & 0.119 \\
\hline$t-b m(10 \%)$ & 0.078 & 0.104 & 0.061 & 0.097 & 0.072 & 0.085 & 0.077 & 0.104 & 0.091 & 0.135 \\
\hline$t-r t(5$ & 0.078 & 0.102 & 0.060 & 0.1 & 0.076 & 0.0 & 0.081 & 0. & 0.094 & 0.130 \\
\hline$t-r t(10 \%)$ & 0.086 & 0.112 & 0.066 & 0.103 & 0.080 & 0.085 & 0.087 & 0.105 & 0.102 & 0.142 \\
\hline \multicolumn{11}{|c|}{ Panel B. Seasonal $A R: \Phi=-0.5$} \\
\hline$A I C$ & 0.056 & 0.066 & 0.050 & 0.0 & 0.021 & 0.057 & 0.054 & 0.0 & 0.058 & 0.072 \\
\hline$M A I C$ & 0.033 & 0.037 & 0.040 & 0.069 & 0.038 & 0.048 & 0.041 & 0.053 & 0.039 & 0.048 \\
\hline$M A I C \_P Q$ & & 0.042 & & 0.069 & & 0.048 & & 0.054 & & 0.053 \\
\hline$B I C$ & 62 & 0.067 & .054 & 0.083 & 0.057 & 0.059 & 0.063 & 0.069 & 0.070 & 0.076 \\
\hline$M B I C$ & 0.035 & 0.042 & 0.042 & 0.074 & 0.041 & 0.049 & 0.043 & 0.056 & 0.042 & 0.054 \\
\hline$M B I C \_P Q$ & & 0.045 & & 0.072 & & 0.049 & & 0.057 & & 0.056 \\
\hline$S A I C$ & 0.088 & 0.088 & 0.079 & 0.096 & 0.075 & 0.072 & 0.079 & 0.090 & 0.077 & 0.096 \\
\hline$S M A I C$ & 0.009 & 0.018 & 0.029 & 0.061 & 0.027 & 0.039 & 0.022 & 0.044 & 0.012 & 0.027 \\
\hline$S M A I C \_P Q$ & & 0.020 & & 0.060 & & 0.037 & & 0.044 & & 0.028 \\
\hline$t-s q(5 \%)$ & 0.061 & 0.073 & 0.054 & 0.082 & 0.051 & 0.060 & 0.058 & 0.069 & 0.062 & 0.077 \\
\hline$t-s q(10 \%)$ & 0.063 & 0.077 & 0.060 & 0.082 & 0.052 & 0.063 & 0.059 & 0.073 & 0.062 & 0.083 \\
\hline$t-b m(5 \%)$ & 0.073 & 0.073 & 0.079 & 0.093 & 0.071 & 0.067 & 0.076 & 0.085 & 0.067 & 0.090 \\
\hline$t-b m(10 \%)$ & 0.089 & 0.090 & 0.088 & 0.096 & 0.075 & 0.074 & 0.082 & 0.093 & 0.081 & 0.104 \\
\hline$t-r t(5 \%)$ & 0.077 & 0.076 & 0.074 & 0.095 & 0.069 & 0.067 & 0.071 & 0.084 & 0.068 & 0.088 \\
\hline$t-r t(10 \%)$ & 0.092 & 0.093 & 0.080 & 0.097 & 0.076 & 0.074 & 0.080 & 0.091 & 0.079 & 0.099 \\
\hline
\end{tabular}

Notes: As for Table 1, except that the DGP has seasonal autoregressive disturbances with (1 $\left.\Phi L^{4}\right) u_{4 t+s}=\varepsilon_{4 t+s}$ and maximum lag given by $k_{\max }=i n t\left[\ell(4 N / 100)^{1 / 4}\right]$ with $\ell=12$. 
Table 4: Size-corrected power of quarterly seasonal unit root tests for white noise disturbances

\begin{tabular}{|c|c|c|c|c|c|c|c|c|c|c|c|}
\hline & \multirow[b]{2}{*}{$c$} & \multicolumn{2}{|c|}{$t_{0}$} & \multicolumn{2}{|c|}{$t_{2}$} & \multicolumn{2}{|c|}{$F_{1}$} & \multicolumn{2}{|c|}{$F_{12}$} & \multicolumn{2}{|c|}{$\bar{F} F_{012}$} \\
\hline & & $O L S$ & $G L S$ & $O L S$ & $G L S$ & $O L S$ & $G L S$ & $O L S$ & $G L S$ & $O L S$ & $G L S$ \\
\hline \multicolumn{12}{|l|}{ Panel A. $\ell=4$} \\
\hline \multirow[t]{3}{*}{$A I C$} & 5 & 0.088 & 0.110 & 0.134 & 0.273 & 0.173 & 0.424 & 0.247 & 0.566 & 0.257 & 0.519 \\
\hline & 10 & 0.192 & 0.289 & 0.365 & 0.635 & 0.570 & 0.863 & 0.761 & 0.960 & 0.793 & 0.963 \\
\hline & 20 & 0.714 & 0.849 & 0.929 & 0.976 & 0.993 & 0.990 & 1.000 & 1.000 & 1.000 & 1.000 \\
\hline \multirow[t]{3}{*}{$M A I C$} & 5 & 0.086 & 0.116 & 0.136 & 0.290 & 0.164 & 0.433 & 0.236 & 0.580 & 0.255 & 0.536 \\
\hline & 10 & 0.188 & 0.302 & 0.359 & 0.654 & 0.562 & 0.861 & 0.748 & 0.952 & 0.774 & 0.956 \\
\hline & 20 & 0.694 & 0.811 & 0.912 & 0.957 & 0.986 & 0.980 & 0.998 & 0.999 & 0.999 & 1.000 \\
\hline \multirow{3}{*}{$M A I C_{-} P Q$} & 5 & & 0.120 & & 0.292 & & 0.436 & & 0.579 & & 0.539 \\
\hline & 10 & & 0.311 & & 0.662 & & 0.866 & & 0.954 & & 0.959 \\
\hline & 20 & & 0.852 & & 0.965 & & 0.984 & & 1.000 & & 1.000 \\
\hline \multirow[t]{3}{*}{$B I C$} & 5 & 0.083 & 0.111 & 0.136 & 0.285 & 0.174 & 0.427 & 0.245 & 0.570 & 0.255 & 0.536 \\
\hline & 10 & 0.191 & 0.288 & 0.367 & 0.658 & 0.581 & 0.872 & 0.771 & 0.962 & 0.797 & 0.968 \\
\hline & 20 & 0.722 & 0.862 & 0.942 & 0.984 & 0.997 & 0.993 & 1.000 & 1.000 & 1.000 & 1.000 \\
\hline \multirow{3}{*}{$M B I C_{-} P Q$} & 5 & 0.090 & 0.118 & 0.133 & 0.282 & 0.164 & 0.438 & 0.238 & 0.580 & 0.253 & 0.541 \\
\hline & 10 & 0.191 & 0.301 & 0.358 & 0.652 & 0.564 & 0.869 & 0.758 & 0.958 & 0.779 & 0.963 \\
\hline & 20 & 0.699 & 0.845 & 0.913 & 0.968 & 0.987 & 0.985 & 0.998 & 1.000 & 0.999 & 1.000 \\
\hline \multirow[t]{3}{*}{$S A I C$} & 5 & 0.085 & 0.116 & 0.144 & 0.271 & 0.191 & 0.405 & 0.247 & 0.560 & 0.257 & 0.496 \\
\hline & 10 & 0.190 & 0.300 & 0.363 & 0.622 & 0.584 & 0.839 & 0.741 & 0.954 & 0.775 & 0.951 \\
\hline & 20 & 0.668 & 0.817 & 0.906 & 0.962 & 0.989 & 0.980 & 0.998 & 1.000 & 0.999 & 0.999 \\
\hline \multirow[t]{3}{*}{$S M A I C$} & 5 & 0.101 & 0.119 & 0.142 & 0.283 & 0.200 & 0.438 & 0.272 & 0.574 & 0.286 & 0.541 \\
\hline & 10 & 0.238 & 0.306 & 0.375 & 0.653 & 0.616 & 0.870 & 0.786 & 0.952 & 0.806 & 0.955 \\
\hline & 20 & 0.789 & 0.836 & 0.936 & 0.971 & 0.994 & 0.989 & 0.999 & 1.000 & 1.000 & 1.000 \\
\hline \multirow[t]{3}{*}{$S M A I C_{-} P Q$} & 5 & & 0.119 & & 0.286 & & 0.435 & & 0.578 & & 0.543 \\
\hline & 10 & & 0.313 & & 0.655 & & 0.868 & & 0.955 & & 0.956 \\
\hline & 20 & & 0.859 & & 0.974 & & 0.988 & & 1.000 & & 1.000 \\
\hline \multirow[t]{3}{*}{$t-s q(5 \%)$} & 5 & 0.081 & 0.116 & 0.141 & 0.269 & 0.178 & 0.412 & 0.255 & 0.567 & 0.253 & 0.510 \\
\hline & 10 & 0.187 & 0.293 & 0.368 & 0.627 & 0.574 & 0.854 & 0.765 & 0.959 & 0.786 & 0.955 \\
\hline & 20 & 0.690 & 0.834 & 0.920 & 0.966 & 0.991 & 0.983 & 0.999 & 1.000 & 0.999 & 0.999 \\
\hline \multirow[t]{3}{*}{$t-s q(10 \%)$} & 5 & 0.084 & 0.118 & 0.138 & 0.270 & 0.180 & 0.402 & 0.258 & 0.562 & 0.262 & 0.504 \\
\hline & 10 & 0.186 & 0.296 & 0.357 & 0.615 & 0.563 & 0.834 & 0.761 & 0.953 & 0.787 & 0.950 \\
\hline & 20 & 0.667 & 0.813 & 0.897 & 0.954 & 0.988 & 0.975 & 0.999 & 0.999 & 0.999 & 0.999 \\
\hline \multirow[t]{3}{*}{$t-b m(10 \%)$} & 5 & 0.085 & 0.116 & 0.143 & 0.272 & 0.180 & 0.403 & 0.255 & 0.565 & 0.259 & 0.500 \\
\hline & 10 & 0.192 & 0.298 & 0.363 & 0.621 & 0.569 & 0.836 & 0.759 & 0.954 & 0.780 & 0.950 \\
\hline & 20 & 0.677 & 0.811 & 0.910 & 0.961 & 0.989 & 0.978 & 0.999 & 1.000 & 0.999 & 0.999 \\
\hline \multirow[t]{3}{*}{$t-r t(10 \%)$} & 5 & 0.086 & 0.117 & 0.145 & 0.272 & 0.193 & 0.405 & 0.246 & 0.561 & 0.257 & 0.497 \\
\hline & 10 & 0.195 & 0.299 & 0.364 & 0.623 & 0.583 & 0.837 & 0.739 & 0.953 & 0.772 & 0.949 \\
\hline & 20 & 0.667 & 0.815 & 0.902 & 0.962 & 0.989 & 0.978 & 0.998 & 0.999 & 0.999 & 0.999 \\
\hline
\end{tabular}


Table 4 (continued)

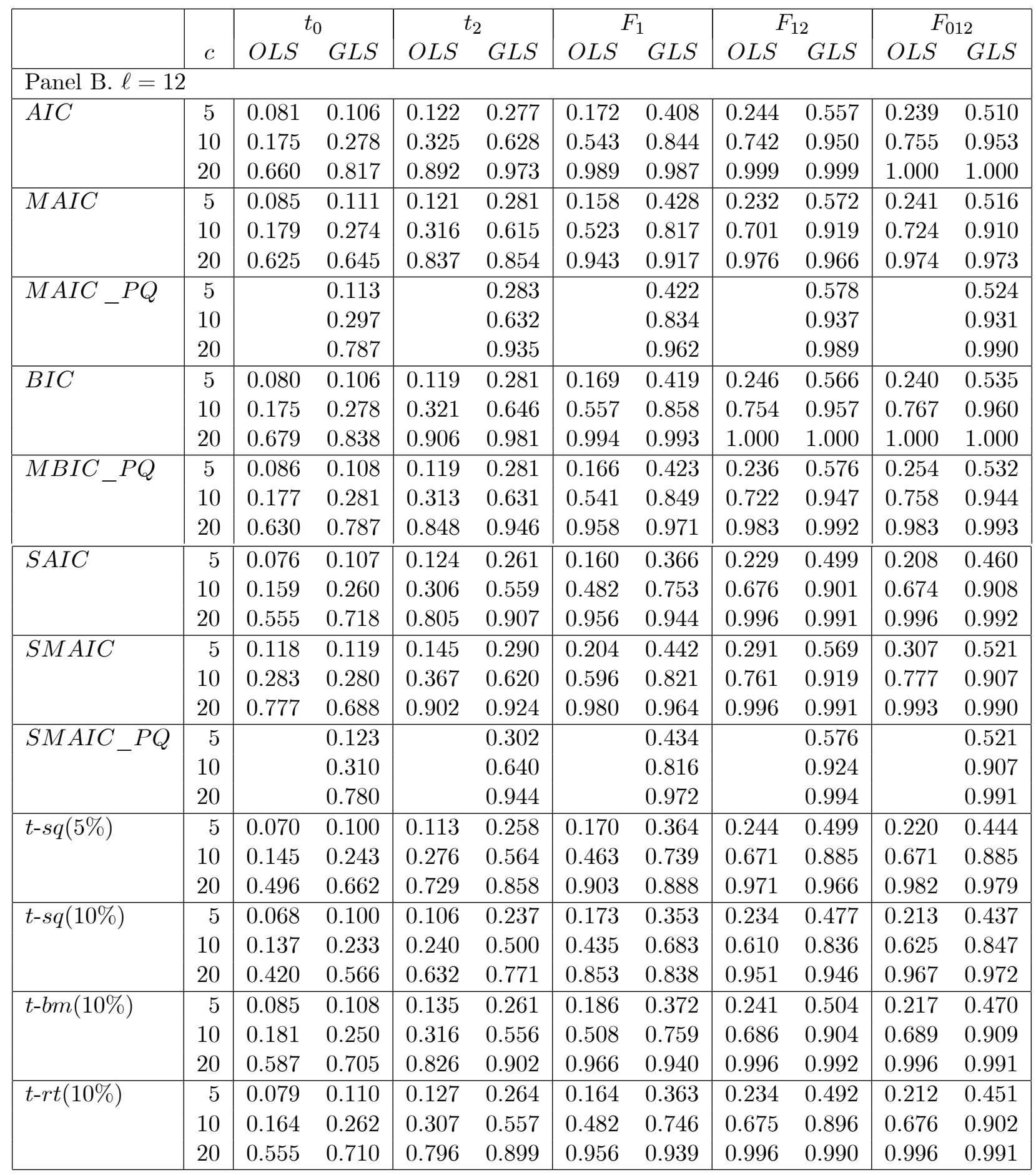

Notes: As for Table 1, except that the DGP is (3.1) with $c=5,10$ and 20. 
Table 5: Size-corrected power of quarterly sesonal unit root tests for moving average disturbances

\begin{tabular}{|c|c|c|c|c|c|c|c|c|c|c|c|}
\hline & \multirow[b]{2}{*}{$c$} & \multicolumn{2}{|c|}{$t_{0}$} & \multicolumn{2}{|c|}{$t_{2}$} & \multicolumn{2}{|c|}{$F_{1}$} & \multicolumn{2}{|c|}{$F_{12}$} & \multicolumn{2}{|c|}{$F_{012}$} \\
\hline & & $O L S$ & $G L S$ & $O L S$ & $G L S$ & $O L S$ & $G L S$ & $O L S$ & $G L S$ & $O L S$ & $G L S$ \\
\hline \multicolumn{12}{|c|}{ Panel A. $M A(1): \theta=-0.8, \Theta=0$} \\
\hline \multirow[t]{3}{*}{$A I C$} & 5 & 0.087 & 0.104 & 0.164 & 0.161 & 0.161 & 0.365 & 0.205 & 0.297 & 0.223 & 0.337 \\
\hline & 10 & 0.179 & 0.239 & 0.398 & 0.334 & 0.472 & 0.692 & 0.589 & 0.587 & 0.640 & 0.675 \\
\hline & 20 & 0.554 & 0.630 & 0.862 & 0.534 & 0.963 & 0.906 & 0.975 & 0.788 & 0.989 & 0.908 \\
\hline \multirow[t]{3}{*}{$M A I C$} & 5 & 0.085 & 0.111 & 0.147 & 0.152 & 0.127 & 0.341 & 0.190 & 0.401 & 0.203 & 0.399 \\
\hline & 10 & 0.158 & 0.225 & 0.328 & 0.290 & 0.359 & 0.631 & 0.502 & 0.687 & 0.554 & 0.716 \\
\hline & 20 & 0.438 & 0.495 & 0.694 & 0.412 & 0.829 & 0.797 & 0.911 & 0.833 & 0.945 & 0.897 \\
\hline \multirow[t]{3}{*}{$M A I C_{-} P Q$} & 5 & & 0.118 & & 0.155 & & 0.355 & & 0.385 & & 0.388 \\
\hline & 10 & & 0.248 & & 0.298 & & 0.658 & & 0.680 & & 0.716 \\
\hline & 20 & & 0.597 & & 0.513 & & 0.854 & & 0.866 & & 0.924 \\
\hline \multirow[t]{3}{*}{$B I C$} & 5 & 0.076 & 0.107 & 0.166 & 0.159 & 0.157 & 0.359 & 0.171 & 0.188 & 0.187 & 0.192 \\
\hline & 10 & 0.148 & 0.253 & 0.404 & 0.342 & 0.457 & 0.690 & 0.451 & 0.454 & 0.516 & 0.500 \\
\hline & 20 & 0.470 & 0.671 & 0.889 & 0.581 & 0.975 & 0.938 & 0.952 & 0.764 & 0.974 & 0.837 \\
\hline \multirow[t]{3}{*}{$M B I C_{-} P Q$} & 5 & 0.083 & 0.123 & 0.140 & 0.156 & 0.131 & 0.367 & 0.191 & 0.374 & 0.205 & 0.394 \\
\hline & 10 & 0.161 & 0.263 & 0.309 & 0.295 & 0.371 & 0.672 & 0.501 & 0.663 & 0.552 & 0.723 \\
\hline & 20 & 0.444 & 0.618 & 0.671 & 0.503 & 0.843 & 0.862 & 0.905 & 0.858 & 0.941 & 0.925 \\
\hline \multirow[t]{3}{*}{$S A I C$} & 5 & 0.081 & 0.101 & 0.175 & 0.165 & 0.159 & 0.329 & 0.226 & 0.304 & 0.239 & 0.305 \\
\hline & 10 & 0.162 & 0.207 & 0.407 & 0.320 & 0.427 & 0.620 & 0.590 & 0.569 & 0.624 & 0.618 \\
\hline & 20 & 0.457 & 0.529 & 0.863 & 0.485 & 0.913 & 0.820 & 0.958 & 0.751 & 0.978 & 0.851 \\
\hline \multirow[t]{3}{*}{$S M A I C$} & 5 & 0.115 & 0.124 & 0.163 & 0.134 & 0.168 & 0.358 & 0.235 & 0.398 & 0.277 & 0.408 \\
\hline & 10 & 0.230 & 0.251 & 0.348 & 0.257 & 0.432 & 0.641 & 0.588 & 0.678 & 0.681 & 0.749 \\
\hline & 20 & 0.521 & 0.536 & 0.720 & 0.377 & 0.846 & 0.805 & 0.951 & 0.833 & 0.982 & 0.939 \\
\hline \multirow[t]{3}{*}{$S M A I C \_P Q$} & 5 & & 0.129 & & 0.142 & & 0.355 & & 0.386 & & 0.408 \\
\hline & 10 & & 0.265 & & 0.283 & & 0.642 & & 0.665 & & 0.749 \\
\hline & 20 & & 0.575 & & 0.478 & & 0.813 & & 0.847 & & 0.940 \\
\hline \multirow[t]{3}{*}{$t-s q(5 \%)$} & 5 & 0.082 & 0.107 & 0.159 & 0.161 & 0.159 & 0.340 & 0.216 & 0.310 & 0.239 & 0.333 \\
\hline & 10 & 0.159 & 0.224 & 0.330 & 0.288 & 0.435 & 0.630 & 0.507 & 0.560 & 0.572 & 0.638 \\
\hline & 20 & 0.450 & 0.528 & 0.658 & 0.410 & 0.883 & 0.806 & 0.875 & 0.727 & 0.934 & 0.845 \\
\hline \multirow[t]{3}{*}{$t-s q(10 \%)$} & 5 & 0.079 & 0.099 & 0.152 & 0.158 & 0.139 & 0.324 & 0.195 & 0.339 & 0.209 & 0.330 \\
\hline & 10 & 0.159 & 0.198 & 0.316 & 0.275 & 0.383 & 0.597 & 0.505 & 0.601 & 0.567 & 0.637 \\
\hline & 20 & 0.413 & 0.452 & 0.649 & 0.393 & 0.821 & 0.765 & 0.900 & 0.752 & 0.945 & 0.847 \\
\hline \multirow[t]{3}{*}{$t$-bm(10\%) } & 5 & 0.084 & 0.098 & 0.178 & 0.166 & 0.162 & 0.337 & 0.236 & 0.310 & 0.255 & 0.322 \\
\hline & 10 & 0.164 & 0.213 & 0.410 & 0.320 & 0.459 & 0.636 & 0.613 & 0.578 & 0.655 & 0.655 \\
\hline & 20 & 0.491 & 0.520 & 0.888 & 0.473 & 0.953 & 0.830 & 0.972 & 0.751 & 0.985 & 0.852 \\
\hline \multirow[t]{3}{*}{$t-r t(10 \%)$} & 5 & 0.083 & 0.101 & 0.176 & 0.167 & 0.156 & 0.333 & 0.230 & 0.302 & 0.245 & 0.320 \\
\hline & 10 & 0.165 & 0.204 & 0.410 & 0.318 & 0.425 & 0.619 & 0.592 & 0.570 & 0.629 & 0.628 \\
\hline & 20 & 0.458 & 0.518 & 0.864 & 0.483 & 0.905 & 0.818 & 0.957 & 0.750 & 0.977 & 0.854 \\
\hline
\end{tabular}


Table 5 (continued)

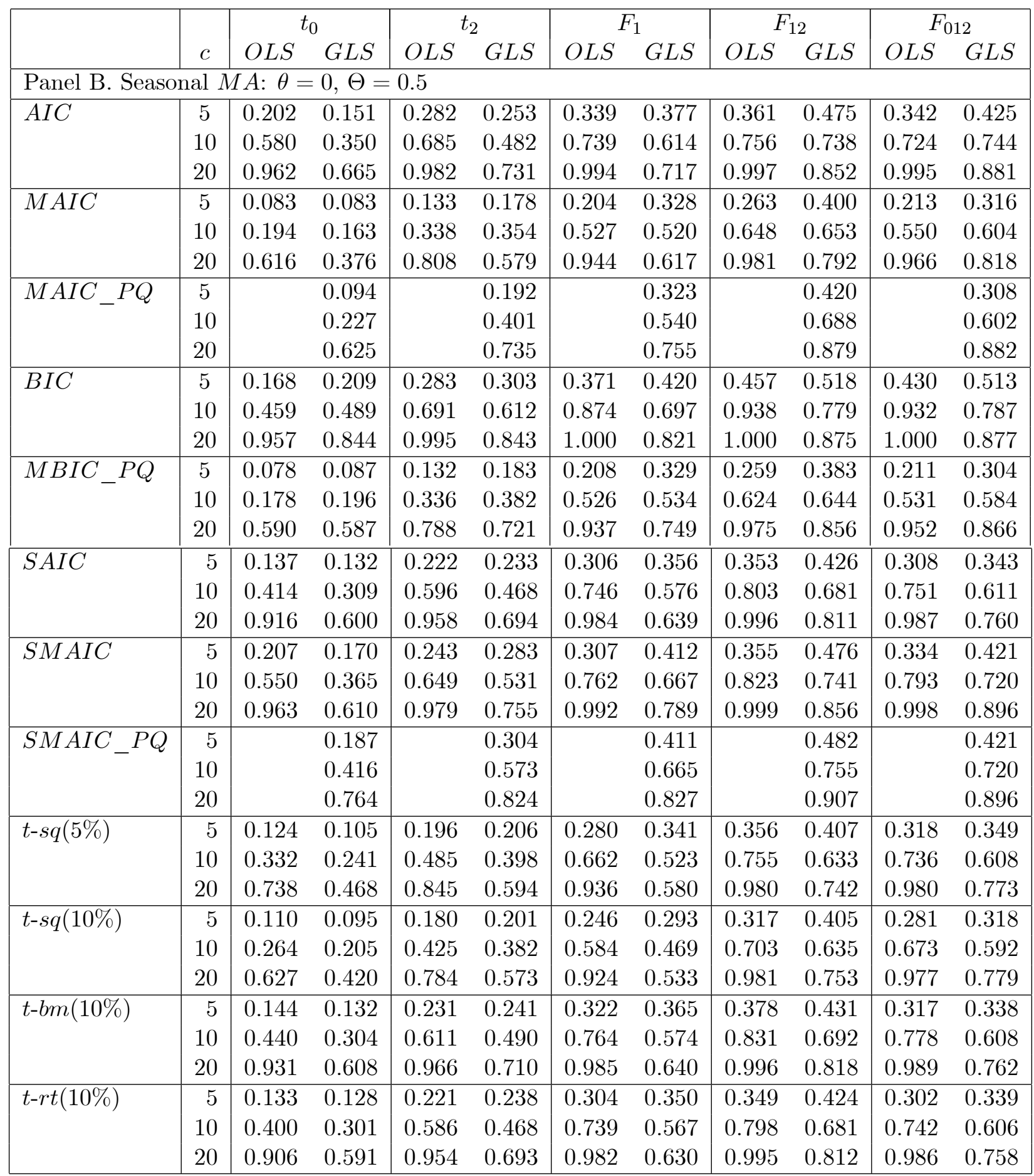


Table 5 (continued)

\begin{tabular}{|c|c|c|c|c|c|c|c|c|c|c|c|}
\hline & \multirow[b]{2}{*}{$c$} & \multicolumn{2}{|c|}{$t_{0}$} & \multicolumn{2}{|c|}{$t_{2}$} & \multicolumn{2}{|c|}{$F_{1}$} & \multicolumn{2}{|c|}{$F_{12}$} & \multicolumn{2}{|c|}{$F_{012}$} \\
\hline & & $O L S$ & $G L S$ & $O L S$ & $G L S$ & $O L S$ & $G L S$ & $O L S$ & $G L S$ & $O L S$ & $G L S$ \\
\hline \multicolumn{12}{|c|}{ Panel C. Seasonal $M A: \theta=0 . \Theta=-0.5$} \\
\hline \multirow{3}{*}{$A I C$} & 5 & 0.097 & 0.114 & 0.137 & 0.242 & 0.179 & 0.413 & 0.236 & 0.539 & 0.244 & 0.449 \\
\hline & 10 & 0.179 & 0.230 & 0.304 & 0.519 & 0.469 & 0.750 & 0.589 & 0.863 & 0.618 & 0.810 \\
\hline & 20 & 0.465 & 0.524 & 0.675 & 0.786 & 0.863 & 0.903 & 0.923 & 0.968 & 0.918 & 0.955 \\
\hline \multirow[t]{3}{*}{$M A I C$} & 5 & 0.059 & 0.086 & 0.068 & 0.226 & 0.091 & 0.344 & 0.105 & 0.473 & 0.105 & 0.400 \\
\hline & 10 & 0.056 & 0.141 & 0.101 & 0.454 & 0.179 & 0.649 & 0.232 & 0.843 & 0.223 & 0.807 \\
\hline & 20 & 0.161 & 0.339 & 0.328 & 0.766 & 0.628 & 0.908 & 0.795 & 0.985 & 0.825 & 0.989 \\
\hline \multirow[t]{3}{*}{$M A I C_{-} P Q$} & 5 & & 0.079 & & 0.218 & & 0.331 & & 0.454 & & 0.373 \\
\hline & 10 & & 0.109 & & 0.412 & & 0.611 & & 0.807 & & 0.753 \\
\hline & 20 & & 0.333 & & 0.766 & & 0.916 & & 0.986 & & 0.990 \\
\hline \multirow[t]{3}{*}{$B I C$} & 5 & 0.098 & 0.113 & 0.141 & 0.258 & 0.193 & 0.489 & 0.259 & 0.605 & 0.263 & 0.511 \\
\hline & 10 & 0.193 & 0.244 & 0.309 & 0.544 & 0.513 & 0.824 & 0.657 & 0.904 & 0.680 & 0.873 \\
\hline & 20 & 0.469 & 0.508 & 0.669 & 0.772 & 0.868 & 0.940 & 0.915 & 0.979 & 0.903 & 0.952 \\
\hline \multirow[t]{3}{*}{$M B I C_{-} P Q$} & 5 & 0.048 & 0.083 & 0.035 & 0.216 & 0.065 & 0.190 & 0.070 & 0.254 & 0.070 & 0.254 \\
\hline & 10 & 0.084 & 0.206 & 0.103 & 0.540 & 0.164 & 0.477 & 0.240 & 0.703 & 0.266 & 0.764 \\
\hline & 20 & 0.377 & 0.711 & 0.505 & 0.939 & 0.714 & 0.924 & 0.915 & 0.994 & 0.956 & 1.000 \\
\hline \multirow[t]{3}{*}{$S A I C$} & 5 & 0.094 & 0.103 & 0.144 & 0.235 & 0.164 & 0.347 & 0.197 & 0.453 & 0.188 & 0.361 \\
\hline & 10 & 0.170 & 0.207 & 0.293 & 0.497 & 0.400 & 0.680 & 0.481 & 0.820 & 0.442 & 0.742 \\
\hline & 20 & 0.434 & 0.466 & 0.665 & 0.793 & 0.816 & 0.889 & 0.885 & 0.969 & 0.853 & 0.954 \\
\hline \multirow[t]{3}{*}{$S M A I C$} & 5 & 0.118 & 0.122 & 0.135 & 0.279 & 0.158 & 0.442 & 0.206 & 0.580 & 0.216 & 0.480 \\
\hline & 10 & 0.171 & 0.259 & 0.231 & 0.571 & 0.334 & 0.780 & 0.420 & 0.888 & 0.431 & 0.775 \\
\hline & 20 & 0.042 & 0.092 & 0.089 & 0.338 & 0.168 & 0.600 & 0.192 & 0.688 & 0.151 & 0.473 \\
\hline \multirow[t]{3}{*}{$S M A I C_{-} P Q$} & 5 & & 0.130 & & 0.290 & & 0.417 & & 0.563 & & 0.480 \\
\hline & 10 & & 0.229 & & 0.523 & & 0.694 & & 0.823 & & 0.775 \\
\hline & 20 & & 0.080 & & 0.315 & & 0.526 & & 0.637 & & 0.473 \\
\hline \multirow[t]{3}{*}{$t-s q(5 \%)$} & 5 & 0.083 & 0.100 & 0.110 & 0.227 & 0.143 & 0.346 & 0.187 & 0.475 & 0.195 & 0.406 \\
\hline & 10 & 0.143 & 0.197 & 0.230 & 0.470 & 0.345 & 0.655 & 0.452 & 0.827 & 0.473 & 0.781 \\
\hline & 20 & 0.337 & 0.412 & 0.520 & 0.738 & 0.728 & 0.859 & 0.864 & 0.967 & 0.876 & 0.968 \\
\hline \multirow[t]{3}{*}{$t-s q(10 \%)$} & 5 & 0.079 & 0.094 & 0.107 & 0.225 & 0.130 & 0.339 & 0.173 & 0.462 & 0.190 & 0.400 \\
\hline & 10 & 0.133 & 0.179 & 0.212 & 0.467 & 0.315 & 0.646 & 0.428 & 0.828 & 0.459 & 0.791 \\
\hline & 20 & 0.297 & 0.384 & 0.480 & 0.720 & 0.694 & 0.847 & 0.859 & 0.966 & 0.890 & 0.972 \\
\hline \multirow{3}{*}{$t-b m(10 \%)$} & 5 & 0.111 & 0.103 & 0.151 & 0.240 & 0.163 & 0.367 & 0.211 & 0.482 & 0.212 & 0.384 \\
\hline & 10 & 0.198 & 0.211 & 0.309 & 0.521 & 0.402 & 0.697 & 0.515 & 0.837 & 0.480 & 0.770 \\
\hline & 20 & 0.499 & 0.486 & 0.689 & 0.805 & 0.826 & 0.897 & 0.912 & 0.976 & 0.891 & 0.965 \\
\hline \multirow[t]{3}{*}{$t-r t(10 \%)$} & 5 & 0.095 & 0.097 & 0.148 & 0.234 & 0.166 & 0.349 & 0.198 & 0.451 & 0.184 & 0.354 \\
\hline & 10 & 0.172 & 0.200 & 0.292 & 0.496 & 0.396 & 0.681 & 0.481 & 0.819 & 0.437 & 0.737 \\
\hline & 20 & 0.430 & 0.450 & 0.658 & 0.790 & 0.814 & 0.884 & 0.889 & 0.969 & 0.853 & 0.953 \\
\hline
\end{tabular}

Notes: As for Table 2, except that the DGP is (3.1) with $c=5,10$ and 20 . 
Table 6: Size-corrected power of quarterly seasonal unit root tests for autoregressive disturbances

\begin{tabular}{|c|c|c|c|c|c|c|c|c|c|c|c|}
\hline & \multirow[b]{2}{*}{$c$} & \multicolumn{2}{|c|}{$t_{0}$} & \multicolumn{2}{|c|}{$t_{2}$} & \multicolumn{2}{|c|}{$F_{1}$} & \multicolumn{2}{|c|}{$F_{12}$} & \multicolumn{2}{|c|}{$F_{012}$} \\
\hline & & $O L S$ & $G L S$ & $O L S$ & $G L S$ & $O L S$ & $G L S$ & $O L S$ & $G L S$ & $O L S$ & $G L S$ \\
\hline \multicolumn{12}{|c|}{ Panel A. Seasonal $A R: \Phi=0.5$} \\
\hline \multirow[t]{3}{*}{$A I C$} & 5 & 0.082 & 0.094 & 0.107 & 0.244 & 0.142 & 0.355 & 0.170 & 0.461 & 0.161 & 0.437 \\
\hline & 10 & 0.141 & 0.197 & 0.225 & 0.492 & 0.333 & 0.712 & 0.445 & 0.856 & 0.454 & 0.856 \\
\hline & 20 & 0.328 & 0.461 & 0.517 & 0.793 & 0.768 & 0.937 & 0.887 & 0.987 & 0.910 & 0.989 \\
\hline \multirow[t]{3}{*}{$M A I C$} & 5 & 0.078 & 0.092 & 0.098 & 0.248 & 0.124 & 0.363 & 0.159 & 0.495 & 0.158 & 0.445 \\
\hline & 10 & 0.089 & 0.162 & 0.147 & 0.443 & 0.228 & 0.661 & 0.322 & 0.804 & 0.315 & 0.769 \\
\hline & 20 & 0.033 & 0.077 & 0.084 & 0.380 & 0.225 & 0.712 & 0.284 & 0.856 & 0.229 & 0.798 \\
\hline \multirow[t]{3}{*}{$M A I C_{-} P Q$} & 5 & & 0.096 & & 0.250 & & 0.363 & & 0.494 & & 0.447 \\
\hline & 10 & & 0.149 & & 0.408 & & 0.622 & & 0.751 & & 0.708 \\
\hline & 20 & & 0.070 & & 0.359 & & 0.697 & & 0.841 & & 0.775 \\
\hline \multirow[t]{3}{*}{$B I C$} & 5 & 0.083 & 0.096 & 0.108 & 0.246 & 0.135 & 0.359 & 0.166 & 0.470 & 0.157 & 0.442 \\
\hline & 10 & 0.143 & 0.201 & 0.219 & 0.488 & 0.331 & 0.710 & 0.443 & 0.845 & 0.447 & 0.839 \\
\hline & 20 & 0.296 & 0.375 & 0.442 & 0.660 & 0.649 & 0.850 & 0.742 & 0.919 & 0.734 & 0.897 \\
\hline \multirow[t]{3}{*}{$M B I C_{-} P Q$} & 5 & 0.015 & 0.016 & 0.018 & 0.066 & 0.055 & 0.148 & 0.041 & 0.161 & 0.031 & 0.117 \\
\hline & 10 & 0.014 & 0.023 & 0.017 & 0.135 & 0.086 & 0.263 & 0.067 & 0.328 & 0.044 & 0.238 \\
\hline & 20 & 0.065 & 0.165 & 0.133 & 0.550 & 0.269 & 0.674 & 0.359 & 0.874 & 0.346 & 0.883 \\
\hline \multirow[t]{3}{*}{$S A I C$} & 5 & 0.074 & 0.090 & 0.116 & 0.222 & 0.140 & 0.301 & 0.181 & 0.418 & 0.158 & 0.370 \\
\hline & 10 & 0.132 & 0.176 & 0.234 & 0.436 & 0.309 & 0.619 & 0.444 & 0.789 & 0.409 & 0.789 \\
\hline & 20 & 0.290 & 0.404 & 0.509 & 0.735 & 0.704 & 0.885 & 0.857 & 0.972 & 0.857 & 0.973 \\
\hline \multirow{3}{*}{$S M A I C$} & 5 & 0.120 & $\begin{array}{l}0.092 \\
\end{array}$ & 0.139 & 0.258 & 0.171 & $\begin{array}{l}0.432 \\
\end{array}$ & 0.224 & 0.546 & 0.234 & 0.486 \\
\hline & 10 & 0.179 & 0.188 & 0.249 & 0.513 & 0.355 & 0.778 & 0.474 & 0.872 & 0.465 & 0.791 \\
\hline & 20 & 0.049 & 0.090 & 0.111 & 0.348 & 0.195 & 0.657 & 0.253 & 0.745 & 0.210 & 0.554 \\
\hline \multirow[t]{3}{*}{$S M A I C \_P Q$} & 5 & & 0.093 & & 0.270 & & 0.401 & & 0.541 & & 0.486 \\
\hline & 10 & & 0.181 & & 0.494 & & 0.721 & & 0.828 & & 0.791 \\
\hline & 20 & & 0.060 & & 0.306 & & 0.568 & & 0.683 & & 0.554 \\
\hline \multirow[t]{3}{*}{$t-s q(5 \%)$} & 5 & 0.077 & 0.086 & 0.107 & 0.225 & 0.139 & 0.325 & 0.166 & 0.448 & 0.165 & 0.390 \\
\hline & 10 & 0.133 & 0.179 & 0.221 & 0.448 & 0.313 & 0.653 & 0.417 & 0.819 & 0.436 & 0.806 \\
\hline & 20 & 0.286 & 0.404 & 0.471 & 0.730 & 0.697 & 0.876 & 0.839 & 0.966 & 0.877 & 0.974 \\
\hline \multirow[t]{3}{*}{$t-s q(10 \%)$} & 5 & 0.076 & 0.084 & 0.107 & 0.216 & 0.137 & 0.306 & 0.162 & 0.423 & 0.163 & 0.377 \\
\hline & 10 & 0.124 & 0.174 & 0.214 & 0.420 & 0.304 & 0.616 & 0.401 & 0.783 & 0.411 & 0.780 \\
\hline & 20 & 0.269 & 0.379 & 0.432 & 0.680 & 0.647 & 0.836 & 0.801 & 0.947 & 0.841 & 0.961 \\
\hline \multirow[t]{3}{*}{$t-b m(10 \%)$} & 5 & 0.085 & 0.091 & 0.122 & 0.223 & 0.143 & 0.319 & 0.186 & 0.446 & 0.171 & 0.376 \\
\hline & 10 & 0.149 & 0.185 & 0.238 & 0.450 & 0.338 & 0.646 & 0.458 & 0.823 & 0.447 & 0.795 \\
\hline & 20 & 0.348 & 0.434 & 0.529 & 0.748 & 0.745 & 0.894 & 0.873 & 0.978 & 0.890 & 0.974 \\
\hline \multirow[t]{3}{*}{$t-r t(10 \%)$} & 5 & 0.077 & 0.089 & 0.115 & 0.227 & 0.140 & 0.299 & 0.180 & 0.413 & 0.161 & 0.369 \\
\hline & 10 & 0.135 & 0.179 & 0.227 & 0.442 & 0.307 & 0.613 & 0.439 & 0.785 & 0.410 & 0.784 \\
\hline & 20 & 0.294 & 0.409 & 0.498 & 0.733 & 0.697 & 0.877 & 0.851 & 0.970 & 0.855 & 0.971 \\
\hline
\end{tabular}


Table 6 (continued)

\begin{tabular}{|c|c|c|c|c|c|c|c|c|c|c|c|}
\hline & \multirow[b]{2}{*}{$c$} & \multicolumn{2}{|c|}{$t_{0}$} & \multicolumn{2}{|c|}{$t_{2}$} & \multicolumn{2}{|c|}{$F_{1}$} & \multicolumn{2}{|c|}{$F_{12}$} & \multicolumn{2}{|c|}{$F_{012}$} \\
\hline & & $O L S$ & $G L S$ & $O L S$ & $G L S$ & $O L S$ & $G L S$ & $O L S$ & $G L S$ & $O L S$ & $G L S$ \\
\hline \multicolumn{12}{|c|}{ Panel B. Seasonal $A R: \Phi=-0.5$} \\
\hline \multirow{3}{*}{$A I C$} & 5 & 0.072 & 0.098 & 0.107 & 0.220 & 0.159 & 0.306 & 0.204 & 0.452 & 0.201 & 0.397 \\
\hline & 10 & 0.164 & 0.237 & 0.287 & 0.479 & 0.503 & 0.620 & 0.667 & 0.819 & 0.688 & 0.834 \\
\hline & 20 & 0.583 & 0.676 & 0.841 & 0.815 & 0.983 & 0.810 & 0.998 & 0.964 & 0.999 & 0.989 \\
\hline \multirow[t]{3}{*}{$M A I C$} & 5 & 0.069 & 0.094 & 0.100 & 0.216 & 0.153 & 0.296 & 0.196 & 0.434 & 0.201 & 0.414 \\
\hline & 10 & 0.158 & 0.226 & 0.270 & 0.434 & 0.490 & 0.587 & 0.635 & 0.762 & 0.653 & 0.779 \\
\hline & 20 & 0.536 & 0.502 & 0.775 & 0.686 & 0.935 & 0.687 & 0.972 & 0.866 & 0.974 & 0.912 \\
\hline \multirow{3}{*}{$M A I C_{-} P Q$} & 5 & & 0.097 & & 0.219 & & 0.302 & & 0.437 & & 0.410 \\
\hline & 10 & & 0.250 & & 0.465 & & 0.609 & & 0.789 & & 0.806 \\
\hline & 20 & & 0.649 & & 0.771 & & 0.766 & & 0.921 & & 0.956 \\
\hline \multirow[t]{3}{*}{$B I C$} & 5 & 0.111 & 0.105 & 0.150 & 0.226 & 0.194 & 0.307 & 0.224 & 0.436 & 0.208 & 0.374 \\
\hline & 10 & 0.278 & 0.262 & 0.386 & 0.488 & 0.527 & 0.626 & 0.648 & 0.812 & 0.642 & 0.815 \\
\hline & 20 & 0.766 & 0.693 & 0.893 & 0.830 & 0.985 & 0.824 & 0.998 & 0.971 & 0.999 & 0.991 \\
\hline \multirow{3}{*}{$M B I C_{-} P Q$} & 5 & 0.072 & 0.100 & 0.103 & 0.216 & 0.158 & 0.307 & 0.196 & 0.441 & 0.206 & 0.414 \\
\hline & 10 & 0.158 & 0.249 & 0.274 & 0.461 & 0.495 & 0.616 & 0.638 & 0.794 & 0.666 & 0.815 \\
\hline & 20 & 0.541 & 0.651 & 0.780 & 0.769 & 0.941 & 0.769 & 0.974 & 0.922 & 0.976 & 0.958 \\
\hline \multirow[t]{3}{*}{$S A I C$} & 5 & 0.094 & 0.113 & 0.136 & 0.231 & 0.207 & 0.329 & 0.230 & 0.444 & 0.206 & 0.370 \\
\hline & 10 & 0.237 & 0.282 & 0.368 & 0.511 & 0.582 & 0.618 & 0.689 & 0.798 & 0.662 & 0.779 \\
\hline & 20 & 0.777 & 0.687 & 0.887 & 0.808 & 0.973 & 0.762 & 0.997 & 0.925 & 0.996 & 0.932 \\
\hline \multirow[t]{3}{*}{$S M A I C$} & 5 & 0.135 & 0.135 & 0.160 & 0.269 & 0.237 & 0.384 & 0.302 & 0.484 & 0.281 & 0.446 \\
\hline & 10 & 0.390 & 0.322 & 0.472 & 0.572 & 0.675 & 0.704 & 0.770 & 0.809 & 0.751 & 0.815 \\
\hline & 20 & 0.901 & 0.631 & 0.955 & 0.820 & 0.990 & 0.841 & 0.997 & 0.917 & 0.994 & 0.940 \\
\hline \multirow[t]{3}{*}{$S M A I C_{-} P Q$} & 5 & & 0.147 & & 0.285 & & 0.391 & & 0.491 & & 0.446 \\
\hline & 10 & & 0.370 & & 0.605 & & 0.711 & & 0.826 & & 0.815 \\
\hline & 20 & & 0.767 & & 0.856 & & 0.861 & & 0.944 & & 0.940 \\
\hline \multirow{3}{*}{$t-s q(5 \%)$} & 5 & 0.072 & 0.102 & 0.104 & 0.218 & 0.157 & 0.286 & 0.199 & 0.417 & 0.205 & 0.376 \\
\hline & 10 & 0.155 & 0.232 & 0.275 & 0.445 & 0.488 & 0.564 & 0.634 & 0.757 & 0.668 & 0.779 \\
\hline & 20 & 0.502 & 0.582 & 0.754 & 0.726 & 0.929 & 0.701 & 0.982 & 0.888 & 0.991 & 0.937 \\
\hline \multirow[t]{3}{*}{$t-s q(10 \%)$} & 5 & 0.072 & 0.096 & 0.100 & 0.214 & 0.144 & 0.265 & 0.189 & 0.387 & 0.202 & 0.354 \\
\hline & 10 & 0.150 & 0.216 & 0.249 & 0.409 & 0.443 & 0.513 & 0.592 & 0.701 & 0.634 & 0.734 \\
\hline & 20 & 0.446 & 0.502 & 0.668 & 0.667 & 0.882 & 0.628 & 0.967 & 0.837 & 0.984 & 0.906 \\
\hline \multirow{3}{*}{$t-b m(10 \%)$} & 5 & 0.100 & 0.113 & 0.136 & 0.255 & 0.197 & 0.334 & 0.219 & 0.450 & 0.203 & 0.380 \\
\hline & 10 & 0.249 & 0.276 & 0.380 & 0.534 & 0.581 & 0.626 & 0.687 & 0.797 & 0.669 & 0.783 \\
\hline & 20 & 0.804 & 0.692 & 0.913 & 0.820 & 0.973 & 0.763 & 0.997 & 0.928 & 0.997 & 0.935 \\
\hline \multirow[t]{3}{*}{$t-r t(10 \%)$} & 5 & 0.093 & 0.110 & 0.136 & 0.225 & 0.207 & 0.323 & 0.227 & 0.435 & 0.204 & 0.363 \\
\hline & 10 & 0.237 & 0.280 & 0.365 & 0.498 & 0.575 & 0.613 & 0.679 & 0.791 & 0.661 & 0.769 \\
\hline & 20 & 0.769 & 0.680 & 0.880 & 0.800 & 0.970 & 0.752 & 0.996 & 0.920 & 0.995 & 0.925 \\
\hline
\end{tabular}

Notes: As for Table 3, except that the DGP is (3.1) with $c=5,10$ and 20 . 


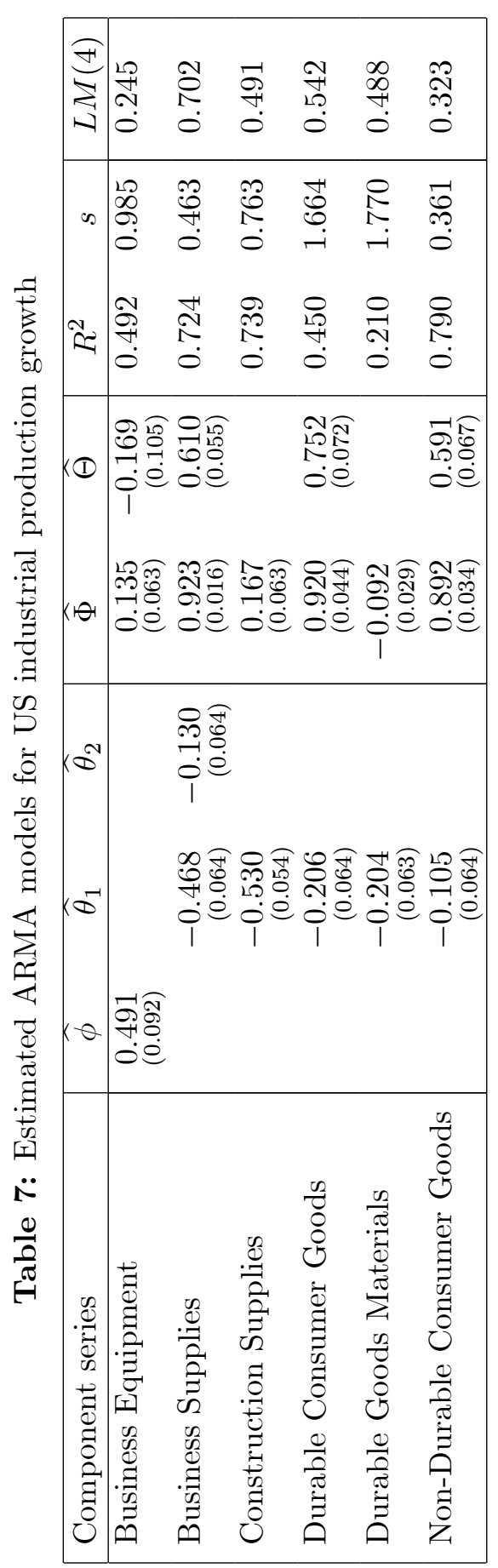

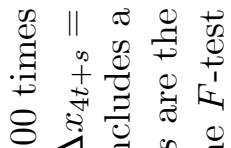
讨.

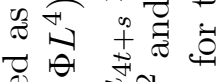

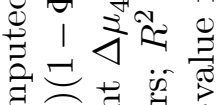
명 存 का

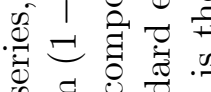

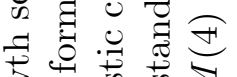

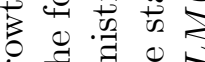
品青署

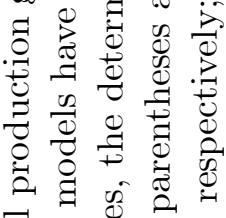

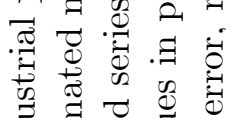

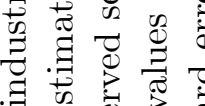

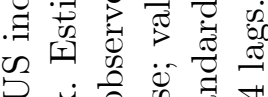
包 .

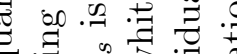

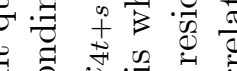
बै.

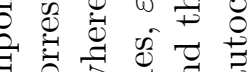
형

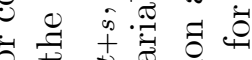

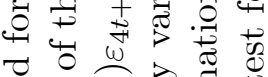

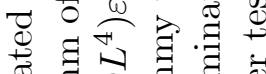
굴

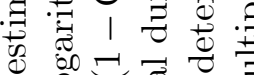
웅 డे की 잉 1100

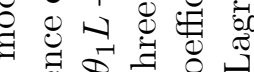
$\varangle 1$ 讨

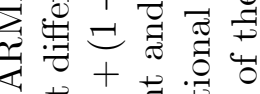

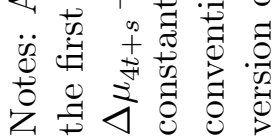




\begin{tabular}{|c|c|c|c|c|}
\hline 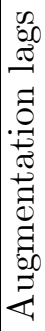 & 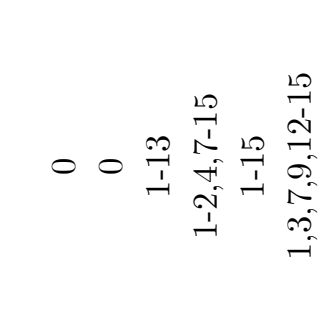 & 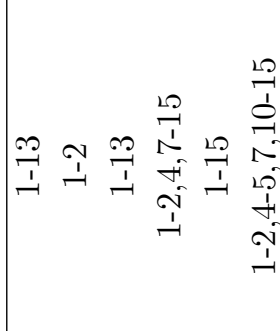 & 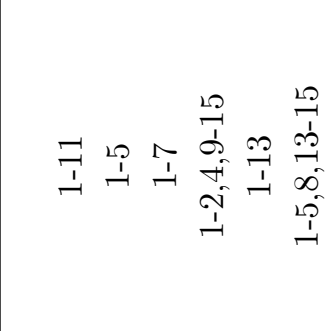 & 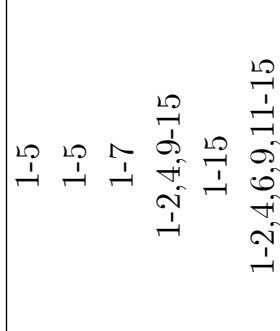 \\
\hline בี & 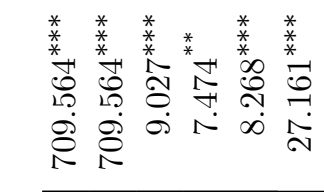 & 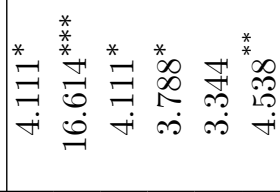 & 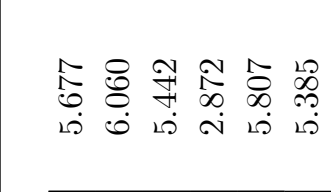 & 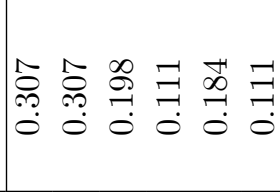 \\
\hline$I^{2}$ & 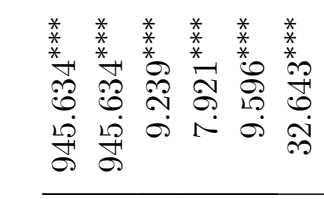 & 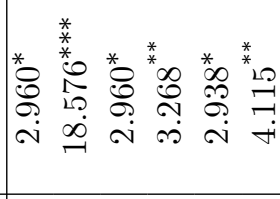 & 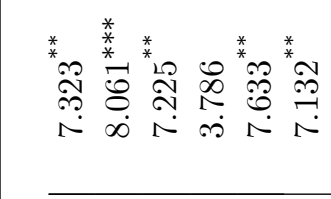 & 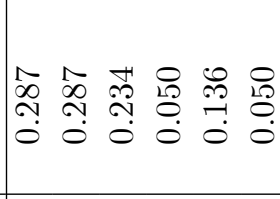 \\
\hline $\mathbb{E}$ & 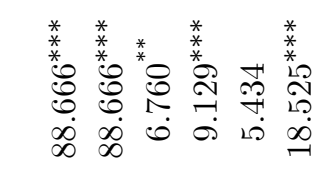 & 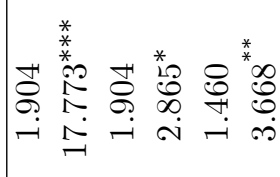 & 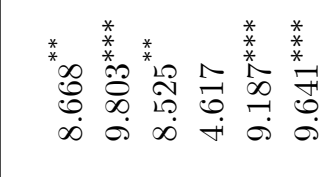 & 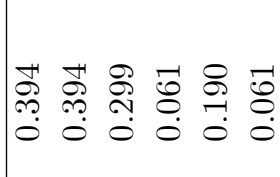 \\
\hline$\stackrel{\sim}{\sim}$ & 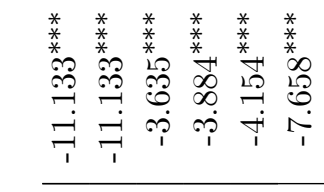 & 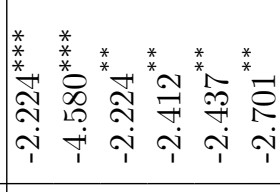 & 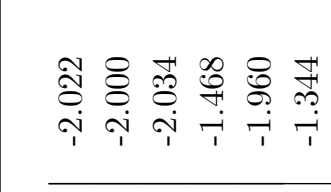 & $\begin{array}{llllll}R & R & 0 & R & 0 & R \\
N & 0 & 0 & 0 & 0 & 0 \\
N & 0 & 0 & 0 \\
i & 0 & 0 & 0 & 0 & 0 \\
1 & 1 & 1 & 1 & 1 & 1 \\
\end{array}$ \\
\hline 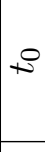 & 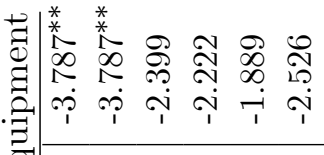 & 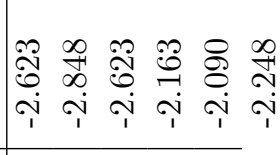 & 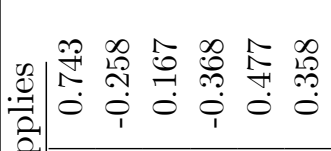 & 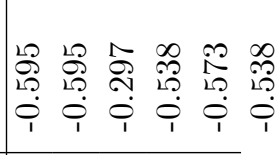 \\
\hline & 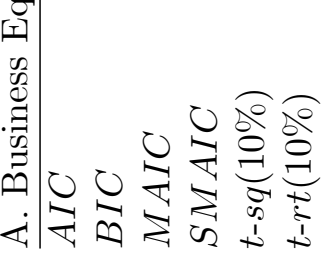 & 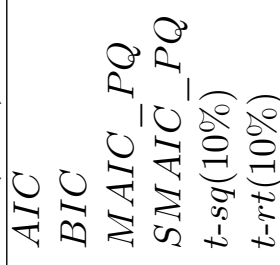 & 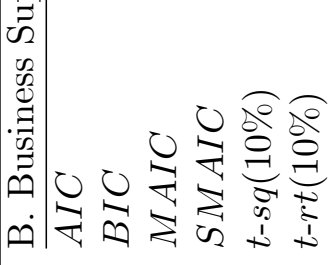 & 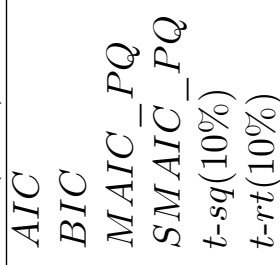 \\
\hline & in & Th & 政 & $\frac{\pi}{6}$ \\
\hline
\end{tabular}




\begin{tabular}{|c|c|c|c|c|}
\hline 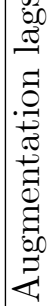 & $00 \frac{0}{30}$ & 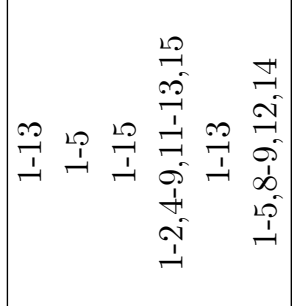 & 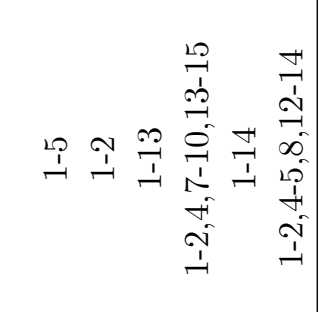 & 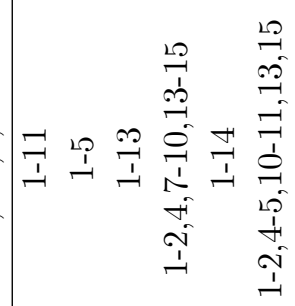 \\
\hline $12^{\circ}$ & 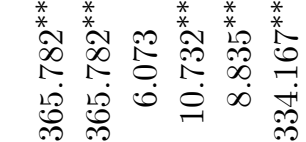 & 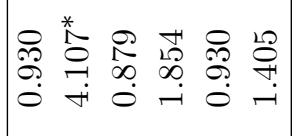 & 管量 & \\
\hline$x^{2}$ & 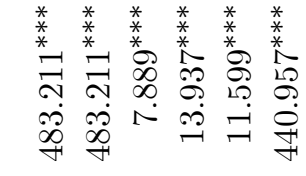 & & 可 & \\
\hline & 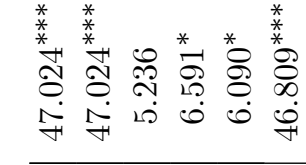 & & $\exists \dot{\square}$ & \\
\hline & 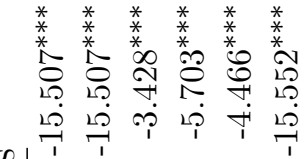 & & & \\
\hline & $\begin{array}{l}0 \\
0\end{array}$ & & 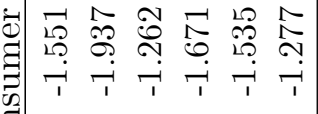 & 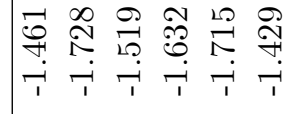 \\
\hline & : & & 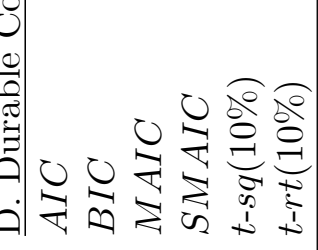 & 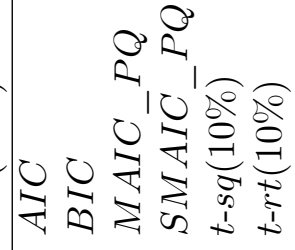 \\
\hline & ñ & 水 & 蛹 & 恐 \\
\hline
\end{tabular}




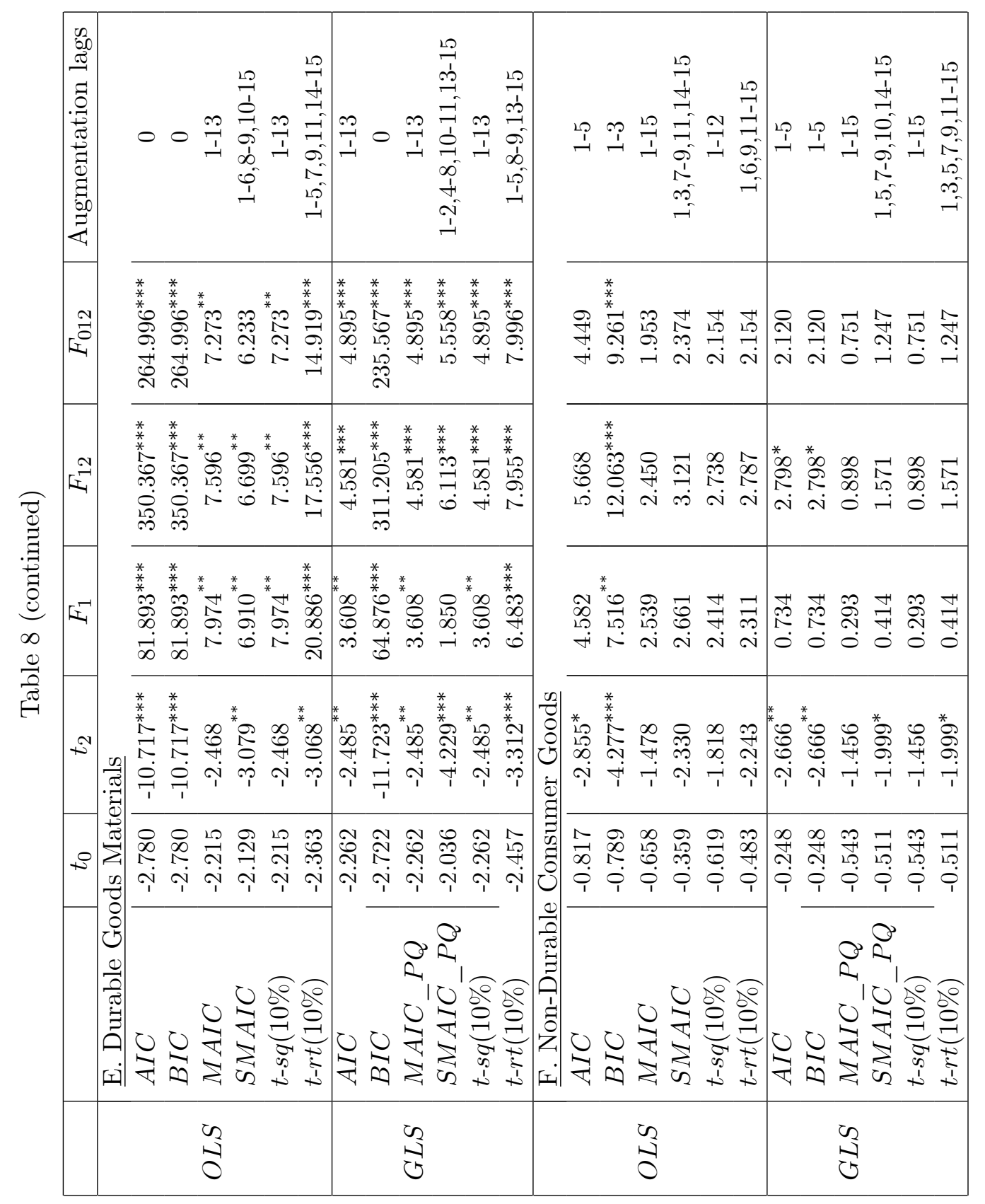




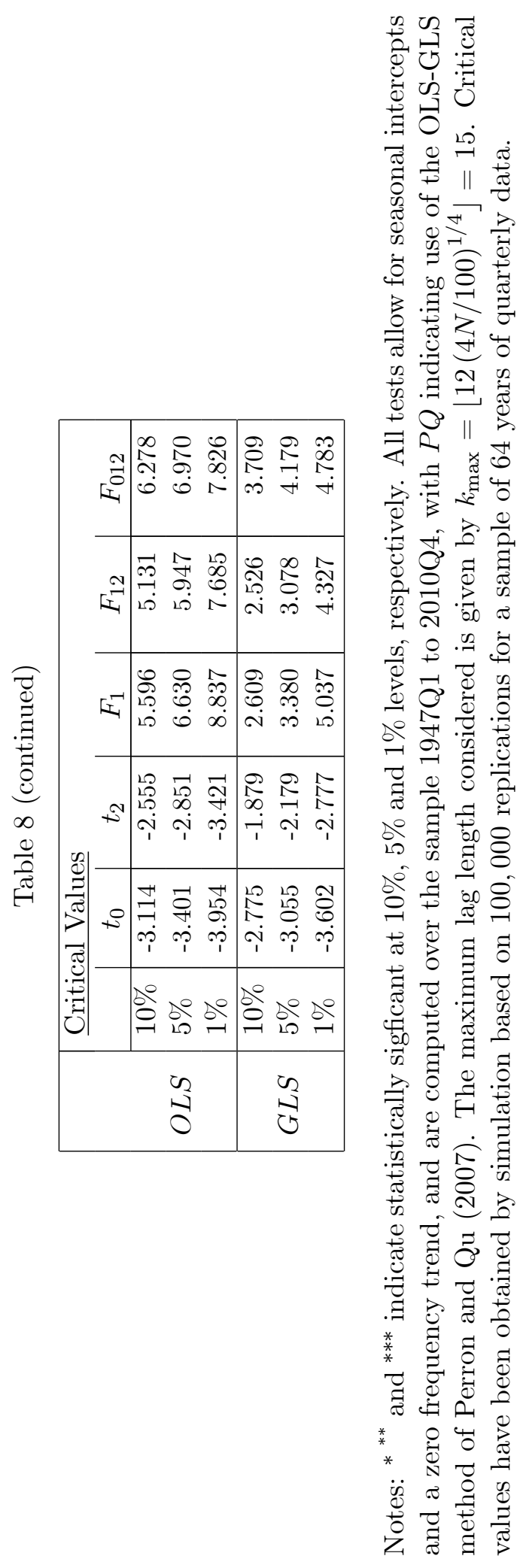




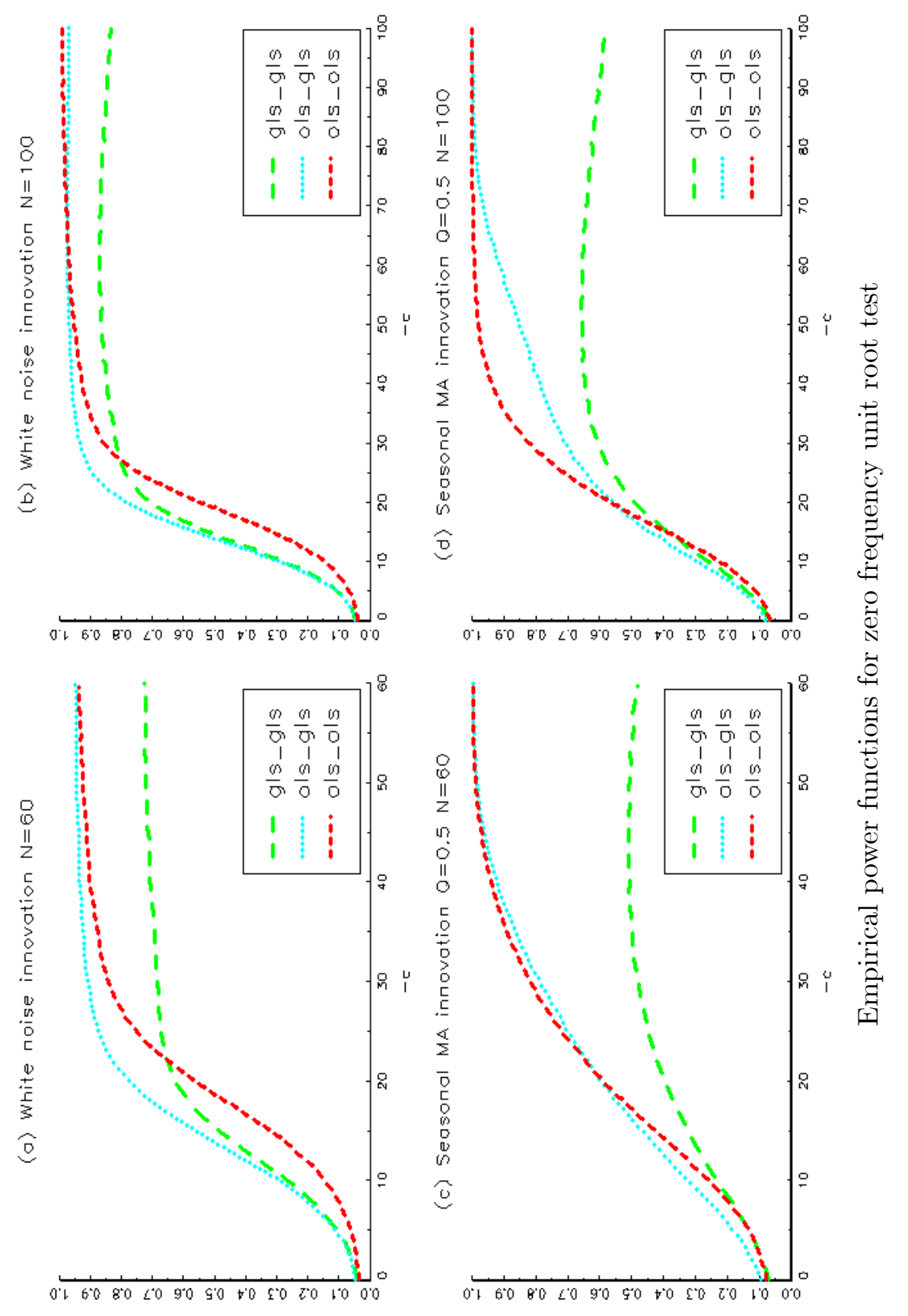



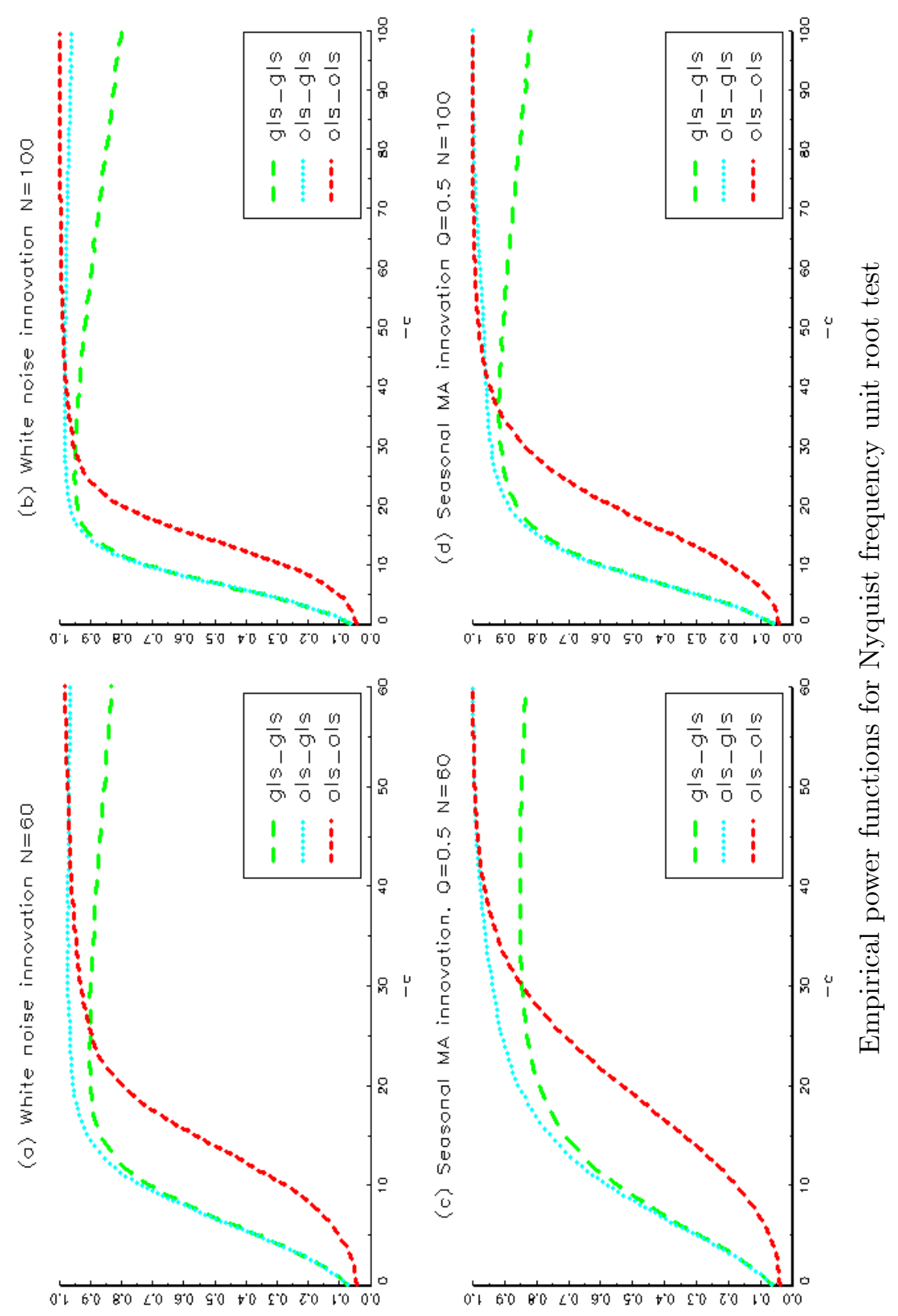

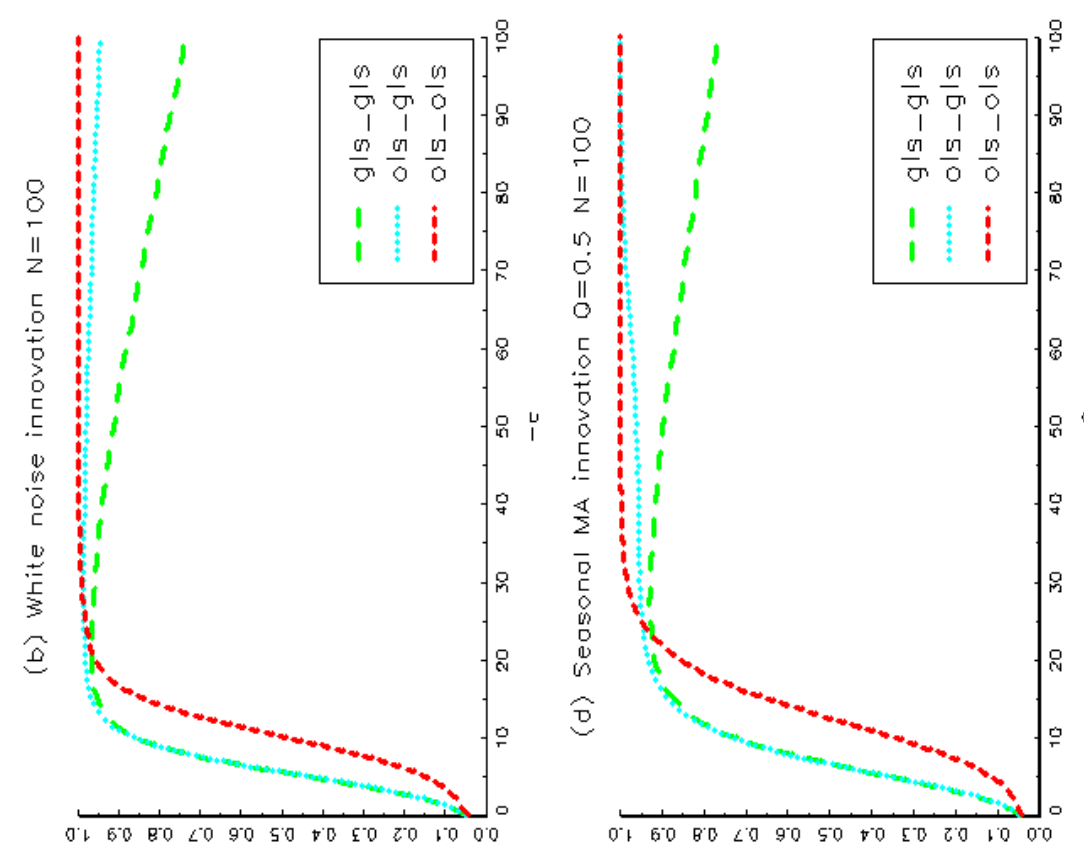

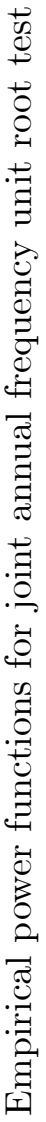
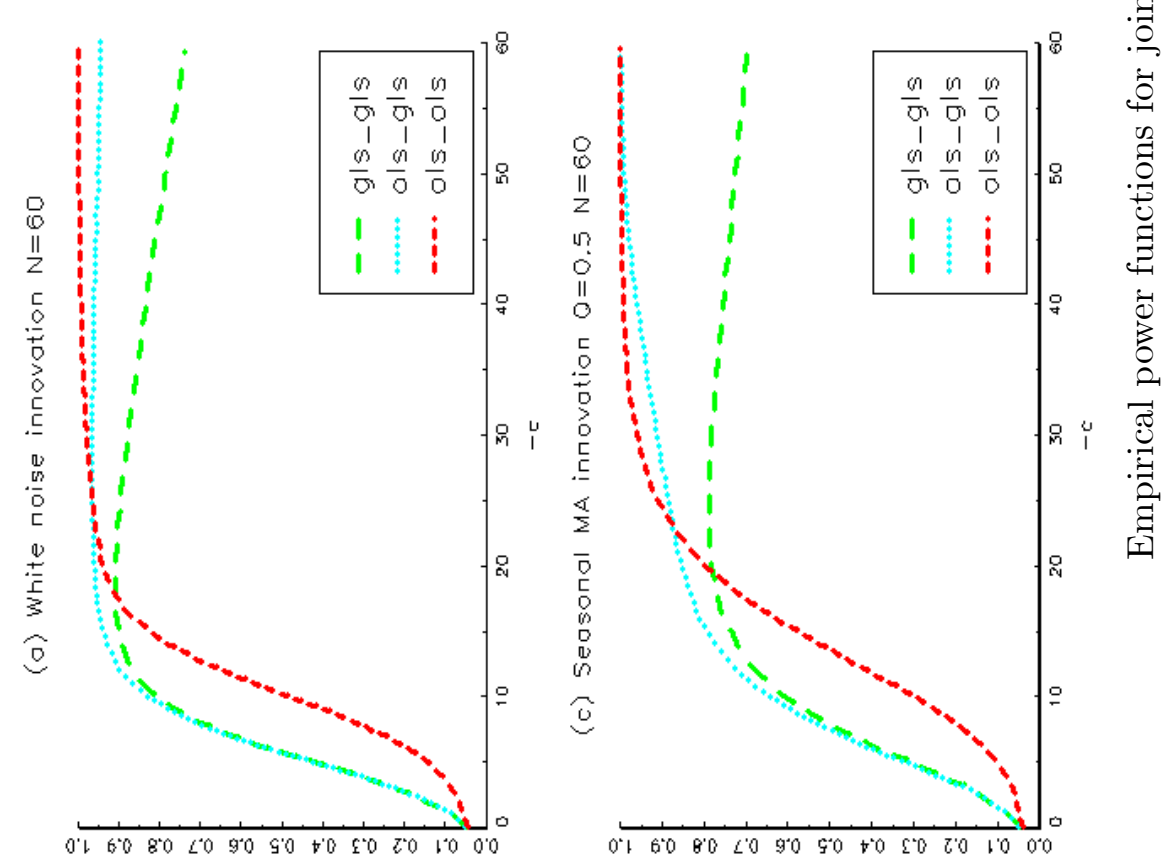

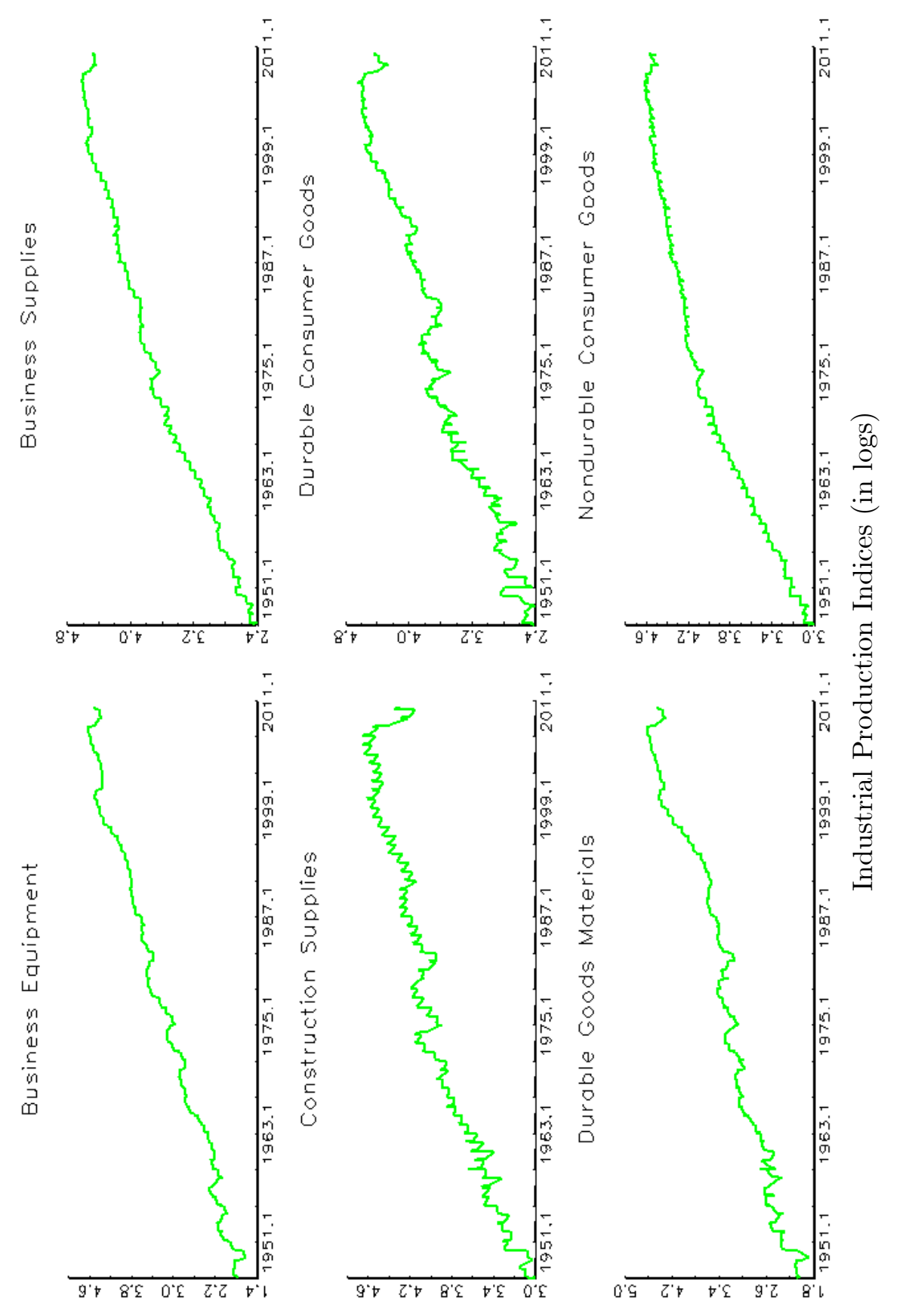
Appendix Table A1: Empirical size of quarterly seasonal unit root tests for white noise disturbances with $N=100$

\begin{tabular}{|c|c|c|c|c|c|c|c|c|c|c|}
\hline & \multicolumn{2}{|c|}{$t_{0}$} & \multicolumn{2}{|c|}{$t_{2}$} & \multicolumn{2}{|c|}{$F_{1}$} & \multicolumn{2}{|c|}{$F_{12}$} & \multicolumn{2}{|c|}{$F_{012}$} \\
\hline & $L S$ & $G L S$ & $O L S$ & $G L S$ & $O L S$ & $G L S$ & $O L S$ & $G L S$ & $O L S$ & $G L S$ \\
\hline \multicolumn{11}{|l|}{ Panel A. $\ell=4$} \\
\hline$A I C$ & 0.050 & 0.063 & .049 & 0.072 & 0.050 & 0.059 & .048 & 0.064 & 0.048 & 0.077 \\
\hline$M A I C$ & 0.040 & 0.049 & 0.046 & 0.066 & 0.041 & 0.052 & 0.042 & 0.056 & 0.040 & 0.062 \\
\hline$M A I C \quad P Q$ & & 0.050 & & 0.066 & & 0.053 & & 0.056 & & 0.062 \\
\hline$B I C$ & 048 & 0.060 & .048 & 0.071 & .045 & 0.058 & 0.046 & 0.062 & 0.044 & 0.073 \\
\hline$M B I C$ & 0.043 & 0.053 & 0.047 & 0.069 & 0.042 & 0.055 & 0.043 & 0.058 & 0.041 & 0.066 \\
\hline$M B I C_{-} P Q$ & & 0.054 & & 0.069 & & 0.055 & & 0.058 & & 0.066 \\
\hline$S A I C$ & 55 & 0.069 & 051 & 0.073 & 049 & 0.060 & $\overline{052}$ & 0.067 & .053 & 0.081 \\
\hline$S M A I C$ & 0.030 & 0.041 & 0.038 & 0.064 & 0.036 & 0.049 & 0.038 & 0.052 & 0.033 & 0.057 \\
\hline$S M A I C \_P Q$ & & 0.042 & & 0.064 & & 0.050 & & 0.053 & & 0.057 \\
\hline$t-s q(5 \%)$ & 0.053 & 0.067 & 0.050 & 0.071 & 0.051 & 0.060 & 0.049 & 0.064 & 0.049 & 0.079 \\
\hline$t$-sq(10\%) & 0.053 & 0.068 & 0.050 & 0.071 & 0.052 & 0.059 & 0.050 & 0.066 & 0.052 & 0.079 \\
\hline$t-b m(5 \%)$ & 0.054 & 0.067 & 0.050 & 0.071 & 0.050 & 0.062 & 0.050 & 0.068 & 0.051 & 0.084 \\
\hline$t-b m(10 \%)$ & 0.057 & 0.069 & 0.052 & 0.072 & 0.054 & 0.061 & 0.051 & 0.070 & 0.055 & 0.085 \\
\hline$t-r t(5 \%)$ & 0.052 & 0.067 & 0.049 & 0.071 & 0.048 & 0.061 & 0.051 & 0.066 & 0.052 & 0.081 \\
\hline$t-r t(10 \%)$ & 0.056 & 0.069 & 0.052 & 0.073 & 0.050 & 0.060 & 0.053 & 0.068 & 0.054 & 0.080 \\
\hline \multicolumn{11}{|l|}{ Panel B. $\ell=12$} \\
\hline$A I C$ & 051 & 0.059 & 0.044 & 0.073 & 0.046 & 0.057 & 0.045 & 0.063 & 0.050 & 0.077 \\
\hline$M A I C$ & 0.040 & 0.044 & 0.041 & 0.067 & 0.039 & 0.050 & 0.039 & 0.050 & 0.042 & 0.056 \\
\hline$M A I C_{-} P Q$ & & 0.045 & & 0.066 & & 0.051 & & 0.051 & & 0.057 \\
\hline$B I C$ & 047 & 0.058 & 0.044 & 0.072 & 0.045 & 0.055 & 0.044 & 0.059 & 0.048 & 0.074 \\
\hline$M B I C$ & 0.044 & 0.051 & 0.043 & 0.069 & 0.042 & 0.054 & 0.041 & 0.054 & 0.045 & 0.065 \\
\hline$M B I C \_P Q$ & & 0.051 & & 0.069 & & 0.053 & & 0.054 & & 0.065 \\
\hline$S A I C$ & 068 & 0.081 & 0.057 & 0.082 & 0.060 & 0.067 & 0.061 & 0.076 & 0.071 & 0.096 \\
\hline$S M A I C$ & 0.011 & 0.023 & 0.024 & 0.051 & 0.022 & 0.033 & 0.019 & 0.034 & 0.014 & 0.032 \\
\hline$S M A I C_{-} P Q$ & & 0.022 & & 0.052 & & 0.036 & & 0.036 & & 0.032 \\
\hline$t-s q(5 \%)$ & 0.059 & 0.069 & 0.049 & 0.078 & 0.051 & 0.061 & 0.050 & 0.064 & 0.058 & 0.084 \\
\hline$t-s q(10 \%)$ & 0.058 & 0.068 & 0.049 & 0.080 & 0.050 & 0.061 & 0.049 & 0.066 & 0.057 & 0.080 \\
\hline$t-b m(5 \%)$ & 0.064 & 0.072 & 0.054 & 0.078 & 0.055 & 0.067 & 0.054 & 0.072 & 0.064 & 0.094 \\
\hline$t-b m(10 \%)$ & 0.073 & 0.082 & 0.059 & 0.080 & 0.063 & 0.070 & 0.061 & 0.080 & 0.075 & 0.103 \\
\hline$t-r t(5 \%)$ & 0.063 & 0.072 & 0.053 & 0.077 & 0.054 & 0.062 & 0.054 & 0.071 & 0.063 & 0.091 \\
\hline$t-r t(10 \%)$ & 0.070 & 0.083 & 0.059 & 0.081 & 0.061 & 0.068 & 0.063 & 0.077 & 0.074 & 0.099 \\
\hline
\end{tabular}

Notes: As for Table 1, except that $N=100$. 
Appendix Table A.2: Empirical size of quarterly seasonal unit root tests for moving average disturbances with $N=100$

\begin{tabular}{|c|c|c|c|c|c|c|c|c|c|c|}
\hline & \multicolumn{2}{|c|}{$t_{0}$} & \multicolumn{2}{|c|}{$\overline{t_{2}}$} & \multicolumn{2}{|c|}{$F_{1}$} & \multicolumn{2}{|c|}{$\overline{F_{12}}$} & \multicolumn{2}{|c|}{$\overline{F_{012}}$} \\
\hline & $O L S$ & $G L S$ & $O L S$ & $G L S$ & $O L S$ & $G L S$ & $O L S$ & $G L S$ & $O L S$ & $G L S$ \\
\hline \multicolumn{11}{|c|}{ Panel A. $M A(1): \theta=-0.8, \Theta=0$} \\
\hline$A I C$ & 0.055 & 0.067 & 0.212 & 0.225 & 0.051 & 0.061 & 158 & 0.160 & 0.147 & 0.145 \\
\hline$M A I C$ & 0.029 & 0.037 & 0.069 & 0.105 & 0.037 & 0.044 & 0.057 & 0.065 & 0.053 & 0.053 \\
\hline$M A I C_{-} P Q$ & & 0.037 & & 0.106 & & 0.046 & & 0.068 & & 0.058 \\
\hline$B I C$ & 559 & 0.069 & 0.387 & 0.372 & 0.064 & 0.066 & 0.304 & 0.275 & 0.280 & 0.250 \\
\hline$M B I C$ & 0.025 & 0.027 & 0.093 & 0.170 & 0.033 & 0.037 & 0.063 & 0.083 & 0.058 & 0.062 \\
\hline$M B I C_{-} P Q$ & & 0.030 & & 0.153 & & 0.041 & & 0.083 & & 0.066 \\
\hline$S A I C$ & 0.069 & 0.078 & 0.173 & 0.182 & 0.062 & 0.063 & .137 & 0.135 & 0.140 & 0.135 \\
\hline$S M A I C$ & 0.011 & 0.023 & 0.056 & 0.106 & 0.028 & 0.038 & 0.040 & 0.061 & 0.030 & 0.044 \\
\hline$S M A I C \_P Q$ & & 0.024 & & 0.100 & & 0.041 & & 0.063 & & 0.047 \\
\hline$t-s q(5 \%)$ & 0.059 & 0.073 & 147 & 0.168 & 0.056 & 0.061 & .117 & 0.122 & 0.109 & 0.117 \\
\hline$t-s q(10 \%)$ & 0.056 & 0.068 & 0.100 & 0.120 & 0.054 & 0.056 & 0.087 & 0.093 & 0.085 & 0.095 \\
\hline$t-b m(5 \%)$ & 0.057 & 0.065 & 0.188 & 0.189 & 0.056 & 0.063 & 0.136 & 0.139 & 0.129 & 0.130 \\
\hline$t-b m(10 \%)$ & 0.056 & 0.064 & 0.156 & 0.161 & 0.053 & 0.063 & 0.122 & 0.124 & 0.113 & 0.118 \\
\hline$t-r t(5 \%)$ & 0.067 & 0.075 & 0.211 & 0.220 & 0.061 & 0.066 & 0.161 & 0.156 & .159 & 0.152 \\
\hline$t-r t(10 \%)$ & 0.070 & 0.076 & 0.167 & 0.175 & 0.061 & 0.063 & 0.131 & 0.132 & 0.135 & 0.133 \\
\hline \multicolumn{11}{|c|}{ Panel B. Seasonal $M A: \theta=0, \Theta=0.5$} \\
\hline$A I C$ & 0.197 & 0.206 & 0.144 & 0.167 & 0.140 & 0.101 & .196 & 0.156 & 285 & 0.257 \\
\hline$M A I C$ & 0.067 & 0.083 & 0.061 & 0.096 & 0.058 & 0.050 & 0.072 & 0.066 & 0.094 & 0.089 \\
\hline$M A I C_{-} P Q$ & & 0.087 & & 0.097 & & 0.051 & & 0.070 & & 0.096 \\
\hline$B I C$ & 304 & 0.323 & 0.222 & 0.248 & 226 & 0.143 & .309 & 0.238 & 0.436 & 0.402 \\
\hline$M B I C$ & 0.087 & 0.121 & 0.077 & 0.130 & 0.069 & 0.060 & 0.089 & 0.090 & 0.126 & 0.136 \\
\hline$M B I C \_P Q$ & & 0.112 & & 0.117 & & 0.056 & & 0.085 & & 0.127 \\
\hline$\overline{S A I C}$ & 131 & 0.122 & 0.124 & 0.129 & 0.119 & 0.084 & 0.143 & 0.115 & 0.149 & 0.141 \\
\hline$S M A I C$ & 0.022 & 0.035 & 0.062 & 0.088 & 0.059 & 0.055 & 0.060 & 0.061 & 0.034 & 0.045 \\
\hline$S M A I C \_P Q$ & & 0.036 & & 0.088 & & 0.055 & & 0.060 & & 0.046 \\
\hline$t-s q(5 \%)$ & 0.112 & 0.129 & 0.084 & 0.114 & 0.082 & 0.068 & 0.106 & 0.095 & 0.145 & 0.142 \\
\hline$t-s q(10 \%)$ & 0.091 & 0.113 & 0.071 & 0.098 & 0.069 & 0.062 & 0.088 & 0.084 & 0.118 & 0.123 \\
\hline$t-b m(5 \%)$ & 0.136 & 0.126 & 0.131 & 0.134 & 0.129 & 0.088 & 0.157 & 0.124 & 0.160 & 0.153 \\
\hline$t-b m(10 \%)$ & 0.140 & 0.130 & 0.119 & 0.127 & 0.117 & 0.085 & 0.142 & 0.113 & 0.152 & 0.145 \\
\hline$t-r t(5 \%)$ & 0.133 & 0.124 & 0.127 & 0.135 & 0.127 & 0.088 & 0.154 & 0.121 & 0.163 & 0.144 \\
\hline$t-r t(10 \%)$ & 0.132 & 0.123 & 0.123 & 0.128 & 0.117 & 0.083 & 0.138 & 0.114 & 0.147 & 0.139 \\
\hline
\end{tabular}


Appendix Table A.2 (continued)

\begin{tabular}{|c|c|c|c|c|c|c|c|c|c|c|}
\hline & \multicolumn{2}{|c|}{$t_{0}$} & \multicolumn{2}{|c|}{$t_{2}$} & \multicolumn{2}{|c|}{$F_{1}$} & \multicolumn{2}{|c|}{$F_{12}$} & \multicolumn{2}{|c|}{$F_{012}$} \\
\hline & $O L S$ & $G L S$ & $O L S$ & $G L S$ & $O L S$ & $G L S$ & $O L S$ & $G L S$ & $O L S$ & $G L S$ \\
\hline \multicolumn{11}{|c|}{ Panel C. Seasonal $M A: \theta=0, \Theta=-0.5$} \\
\hline$A I C$ & 0.061 & 0.068 & 0.065 & 0.078 & 0.060 & 0.064 & 0.062 & 0.072 & 0.077 & 0.084 \\
\hline$M A I C$ & 0.027 & 0.026 & 0.041 & 0.051 & 0.034 & 0.044 & 0.034 & 0.043 & 0.032 & 0.042 \\
\hline$M A I C_{-} P Q$ & & 0.025 & & 0.051 & & 0.045 & & 0.043 & & 0.041 \\
\hline$B I C$ & 0.107 & 0.126 & 0.094 & 0.119 & 0.074 & 0.065 & 0.090 & 0.094 & 0.129 & 0.143 \\
\hline$M B I C$ & 0.018 & 0.029 & 0.031 & 0.056 & 0.029 & 0.038 & 0.028 & 0.040 & 0.023 & 0.038 \\
\hline$M B I C \_P Q$ & & 0.015 & & 0.039 & & 0.041 & & 0.037 & & 0.029 \\
\hline$S A I C$ & 0.074 & 0.082 & 0.066 & 0.083 & 0.064 & 0.071 & 0.069 & 0.081 & 0.074 & 0.105 \\
\hline$S M A I C$ & 0.007 & 0.018 & 0.031 & 0.048 & 0.020 & 0.034 & 0.020 & 0.034 & 0.012 & 0.027 \\
\hline$S M A I C \_P Q$ & & 0.018 & & 0.047 & & 0.034 & & 0.034 & & 0.028 \\
\hline$t-s q(5 \%)$ & 0.053 & 0.064 & 0.058 & 0.071 & 0.052 & 0.062 & 0.054 & 0.064 & 0.061 & 0.076 \\
\hline$t-s q(10 \%)$ & 0.052 & 0.065 & 0.058 & 0.073 & 0.050 & 0.059 & 0.052 & 0.064 & 0.058 & 0.080 \\
\hline$t-b m(5 \%)$ & 0.066 & 0.072 & 0.062 & 0.080 & 0.059 & 0.071 & 0.065 & 0.079 & 0.070 & 0.091 \\
\hline$t-b m(10 \%)$ & 0.070 & 0.078 & 0.064 & 0.081 & 0.062 & 0.072 & 0.067 & 0.081 & 0.074 & 0.103 \\
\hline$t-r t(5 \%)$ & 0.069 & 0.080 & 0.066 & 0.083 & 0.064 & 0.069 & 0.067 & 0.078 & 0.071 & 0.098 \\
\hline$t-r t(10 \%)$ & 0.075 & 0.083 & 0.066 & 0.084 & 0.065 & 0.071 & 0.071 & 0.082 & 0.076 & 0.106 \\
\hline
\end{tabular}

Notes: As for Table 2, except that $N=100$. 
Appendix Table A.3: Empirical size of quarterly seasonal unit root tests for autoregressive disturbances with $N=100$

\begin{tabular}{|c|c|c|c|c|c|c|c|c|c|c|}
\hline & \multicolumn{2}{|c|}{$t_{0}$} & \multicolumn{2}{|c|}{$t_{2}$} & \multicolumn{2}{|c|}{$\overline{F_{1}}$} & \multicolumn{2}{|c|}{$\overline{F_{12}}$} & \multicolumn{2}{|c|}{$\overline{F_{012}}$} \\
\hline & $O L S$ & $G L S$ & $O L S$ & $G L S$ & $O L S$ & $G L S$ & $O L S$ & $G L S$ & $O L S$ & $G L S$ \\
\hline \multicolumn{11}{|c|}{ Panel A. Seasonal $A R: \Phi=0.5$} \\
\hline$A I C$ & 0.047 & 0.063 & 0.050 & 0.073 & 0.049 & 0.054 & 0.050 & 0.062 & 0.051 & 0.072 \\
\hline$M A I C$ & 0.035 & 0.047 & 0.043 & 0.067 & 0.043 & 0.046 & 0.041 & 0.051 & 0.042 & 0.051 \\
\hline$M A I C \quad P Q$ & & 0.048 & & 0.066 & & 0.047 & & 0.052 & & 0.053 \\
\hline$B I C$ & 0.045 & 0.060 & 0.048 & 0.072 & 0.048 & 0.052 & 0.047 & 0.060 & 0.049 & 0.069 \\
\hline$M B I C$ & 0.034 & 0.052 & 0.041 & 0.068 & 0.041 & 0.048 & 0.039 & 0.054 & 0.038 & 0.054 \\
\hline$M B I C \_P Q$ & & 0.047 & & 0.064 & & 0.047 & & 0.051 & & 0.049 \\
\hline$S A I C$ & 0.072 & 0.089 & 0.064 & 0.082 & 0.066 & 0.073 & 0.069 & 0.084 & 0.084 & 0.108 \\
\hline$S M A I C$ & 0.011 & 0.019 & 0.026 & 0.056 & 0.020 & 0.031 & 0.019 & 0.032 & 0.015 & 0.028 \\
\hline$S M A I C \_P Q$ & & 0.022 & & 0.057 & & 0.033 & & 0.032 & & 0.031 \\
\hline$t-s q(5 \%)$ & 0.052 & 0.069 & 0.052 & 0.073 & 0.051 & 0.057 & 0.052 & 0.064 & 0.059 & 0.078 \\
\hline$t-s q(10 \%)$ & 0.053 & 0.068 & 0.054 & 0.072 & 0.053 & 0.059 & 0.052 & 0.067 & 0.062 & 0.080 \\
\hline$t-b m(5 \%)$ & 0.057 & 0.072 & 0.057 & 0.077 & 0.058 & 0.064 & 0.059 & 0.075 & 0.064 & 0.091 \\
\hline$t-b m(10 \%)$ & 0.064 & 0.078 & 0.059 & 0.077 & 0.060 & 0.070 & 0.065 & 0.080 & 0.074 & 0.099 \\
\hline$t-r t(5 \%)$ & 0.066 & 0.083 & 0.063 & 0.083 & 0.060 & 0.068 & 0.065 & 0.080 & 0.075 & 0.099 \\
\hline$t-r t(10 \%)$ & 0.073 & 0.090 & 0.064 & 0.082 & 0.067 & 0.073 & 0.071 & 0.084 & 0.086 & 0.109 \\
\hline \multicolumn{11}{|c|}{ Panel B. Seasonal $A R: \Phi=-0.5$} \\
\hline$A I C$ & 0.046 & 0.056 & 0.047 & 0.066 & 0.054 & 0.057 & 0.055 & 0.059 & 0.053 & 0.066 \\
\hline$M A I C$ & 0.030 & 0.040 & 0.040 & 0.062 & 0.047 & 0.050 & 0.047 & 0.050 & 0.041 & 0.050 \\
\hline$M A I C_{-} P Q$ & & 0.040 & & 0.062 & & 0.051 & & 0.051 & & 0.051 \\
\hline$B I C$ & 0.042 & 0.053 & 0.046 & 0.066 & 0.053 & 0.056 & 0.053 & 0.059 & 0.051 & 0.064 \\
\hline$M B I C$ & 0.033 & 0.043 & 0.043 & 0.064 & 0.049 & 0.053 & 0.050 & 0.054 & 0.044 & 0.054 \\
\hline$M B I C_{-} P Q$ & & 0.044 & & 0.064 & & 0.053 & & 0.054 & & 0.055 \\
\hline$S A I C$ & 0.070 & 0.074 & 0.067 & 0.080 & 0.072 & 0.070 & 0.075 & 0.076 & 0.067 & 0.081 \\
\hline$S M A I C$ & 0.010 & 0.021 & 0.031 & 0.056 & 0.036 & 0.042 & 0.032 & 0.044 & 0.017 & 0.029 \\
\hline$S M A I C \_P Q$ & & 0.021 & & 0.054 & & 0.043 & & 0.044 & & 0.030 \\
\hline$t-s q(5 \%)$ & 0.053 & 0.064 & 0.049 & 0.069 & 0.056 & 0.061 & 0.059 & 0.063 & 0.058 & 0.072 \\
\hline$t-s q(10 \%)$ & 0.053 & 0.067 & 0.049 & 0.073 & 0.056 & 0.061 & 0.059 & 0.065 & 0.058 & 0.075 \\
\hline$t-b m(5 \%)$ & 0.061 & 0.064 & 0.064 & 0.077 & 0.069 & 0.069 & 0.070 & 0.077 & 0.061 & 0.078 \\
\hline$t-b m(10 \%)$ & 0.073 & 0.072 & 0.067 & 0.082 & 0.070 & 0.071 & 0.073 & 0.079 & 0.072 & 0.088 \\
\hline$t-r t(5 \%)$ & 0.063 & 0.062 & 0.062 & 0.077 & 0.068 & 0.067 & 0.069 & 0.075 & 0.062 & 0.073 \\
\hline$t-r t(10 \%)$ & 0.072 & 0.074 & 0.069 & 0.082 & 0.073 & 0.071 & 0.078 & 0.076 & 0.071 & 0.082 \\
\hline
\end{tabular}

Notes: As for Table 3, except that $N=100$. 
Appendix Table A.4: Size-corrected power of quarterly seasonal unit root tests for white noise disturbances with $N=100$

\begin{tabular}{|c|c|c|c|c|c|c|c|c|c|c|c|}
\hline & \multirow[b]{2}{*}{$c$} & \multicolumn{2}{|c|}{$t_{0}$} & \multicolumn{2}{|c|}{$t_{2}$} & \multicolumn{2}{|c|}{$F_{1}$} & \multicolumn{2}{|c|}{$F_{12}$} & \multicolumn{2}{|c|}{$\bar{F} F_{012}$} \\
\hline & & $O L S$ & $G L S$ & $O L S$ & $G L S$ & $O L S$ & $G L S$ & $O L S$ & $G L S$ & $O L S$ & $G L S$ \\
\hline \multicolumn{12}{|l|}{ Panel A. $\ell=4$} \\
\hline \multirow[t]{3}{*}{$A I C$} & 5 & 0.081 & 0.109 & 0.118 & 0.262 & 0.195 & 0.449 & 0.264 & 0.592 & 0.268 & 0.521 \\
\hline & 10 & 0.195 & 0.302 & 0.323 & 0.663 & 0.555 & 0.870 & 0.749 & 0.974 & 0.795 & 0.968 \\
\hline & 20 & 0.664 & 0.843 & 0.891 & 0.971 & 0.994 & 0.995 & 1.000 & 1.000 & 1.000 & 1.000 \\
\hline \multirow[t]{3}{*}{$M A I C$} & 5 & 0.086 & 0.115 & 0.114 & 0.272 & 0.191 & 0.460 & 0.253 & 0.604 & 0.267 & 0.548 \\
\hline & 10 & 0.199 & 0.321 & 0.308 & 0.668 & 0.552 & 0.876 & 0.733 & 0.974 & 0.781 & 0.974 \\
\hline & 20 & 0.675 & 0.833 & 0.877 & 0.967 & 0.989 & 0.993 & 1.000 & 0.999 & 1.000 & 1.000 \\
\hline \multirow[t]{3}{*}{$M A I C_{-} P Q$} & 5 & & 0.118 & & 0.270 & & 0.458 & & 0.604 & & 0.550 \\
\hline & 10 & & 0.326 & & 0.670 & & 0.875 & & 0.975 & & 0.976 \\
\hline & 20 & & 0.850 & & 0.970 & & 0.995 & & 0.999 & & 1.000 \\
\hline \multirow[t]{3}{*}{$B I C$} & 5 & 0.085 & 0.109 & 0.118 & 0.267 & 0.204 & 0.458 & 0.268 & 0.601 & 0.274 & 0.540 \\
\hline & 10 & 0.203 & 0.307 & 0.325 & 0.671 & 0.575 & 0.880 & 0.759 & 0.974 & 0.803 & 0.974 \\
\hline & 20 & 0.689 & 0.853 & 0.900 & 0.976 & 0.996 & 0.997 & 1.000 & 1.000 & 1.000 & 1.000 \\
\hline \multirow{3}{*}{$M B I C_{-} P Q$} & 5 & 0.085 & 0.113 & 0.116 & 0.266 & 0.197 & 0.458 & 0.260 & 0.610 & 0.270 & 0.547 \\
\hline & 10 & 0.201 & 0.317 & 0.314 & 0.667 & 0.561 & 0.878 & 0.744 & 0.977 & 0.784 & 0.977 \\
\hline & 20 & 0.674 & 0.843 & 0.881 & 0.969 & 0.991 & 0.995 & 1.000 & 0.999 & 1.000 & 1.000 \\
\hline \multirow[t]{3}{*}{$S A I C$} & 5 & 0.087 & 0.104 & 0.124 & 0.270 & 0.207 & 0.435 & 0.265 & 0.586 & 0.263 & 0.524 \\
\hline & 10 & 0.200 & 0.288 & 0.320 & 0.662 & 0.559 & 0.856 & 0.736 & 0.973 & 0.781 & 0.965 \\
\hline & 20 & 0.650 & 0.806 & 0.869 & 0.963 & 0.991 & 0.993 & 0.999 & 0.999 & 1.000 & 1.000 \\
\hline \multirow[t]{3}{*}{$S M A I C$} & 5 & 0.093 & 0.120 & 0.130 & 0.283 & 0.208 & 0.458 & 0.276 & 0.608 & 0.293 & 0.541 \\
\hline & 10 & 0.222 & 0.339 & 0.338 & 0.687 & 0.587 & 0.882 & 0.761 & 0.976 & 0.801 & 0.972 \\
\hline & 20 & 0.736 & 0.864 & 0.907 & 0.972 & 0.995 & 0.996 & 1.000 & 1.000 & 1.000 & 1.000 \\
\hline \multirow[t]{3}{*}{$S M A I C C_{-} P Q$} & 5 & & 0.120 & & 0.285 & & 0.454 & & 0.605 & & 0.546 \\
\hline & 10 & & 0.339 & & 0.690 & & 0.880 & & 0.977 & & 0.974 \\
\hline & 20 & & 0.866 & & 0.975 & & 0.995 & & 1.000 & & 1.000 \\
\hline \multirow[t]{3}{*}{$t-s q(5 \%)$} & 5 & 0.083 & 0.108 & 0.118 & 0.265 & 0.197 & 0.439 & 0.264 & 0.578 & 0.273 & 0.525 \\
\hline & 10 & 0.192 & 0.289 & 0.318 & 0.663 & 0.548 & 0.859 & 0.745 & 0.971 & 0.793 & 0.967 \\
\hline & 20 & 0.646 & 0.812 & 0.878 & 0.964 & 0.992 & 0.993 & 1.000 & 0.999 & 1.000 & 1.000 \\
\hline \multirow[t]{3}{*}{$t-s q(10 \%)$} & 5 & 0.085 & 0.107 & 0.120 & 0.261 & 0.193 & 0.432 & 0.267 & 0.574 & 0.265 & 0.523 \\
\hline & 10 & 0.193 & 0.287 & 0.313 & 0.651 & 0.536 & 0.851 & 0.736 & 0.970 & 0.777 & 0.964 \\
\hline & 20 & 0.629 & 0.794 & 0.860 & 0.956 & 0.987 & 0.992 & 0.999 & 0.999 & 1.000 & 1.000 \\
\hline \multirow[t]{3}{*}{$t-b m(10 \%)$} & 5 & 0.082 & 0.105 & 0.122 & 0.267 & 0.196 & 0.435 & 0.272 & 0.579 & 0.266 & 0.520 \\
\hline & 10 & 0.197 & 0.290 & 0.316 & 0.658 & 0.546 & 0.856 & 0.746 & 0.971 & 0.778 & 0.964 \\
\hline & 20 & 0.640 & 0.804 & 0.864 & 0.961 & 0.990 & 0.994 & 1.000 & 0.999 & 1.000 & 1.000 \\
\hline \multirow[t]{3}{*}{$t-r t(10 \%)$} & 5 & 0.085 & 0.105 & 0.126 & 0.265 & 0.208 & 0.435 & 0.264 & 0.578 & 0.255 & 0.522 \\
\hline & 10 & 0.200 & 0.289 & 0.315 & 0.659 & 0.558 & 0.854 & 0.732 & 0.971 & 0.771 & 0.964 \\
\hline & 20 & 0.643 & 0.803 & 0.863 & 0.961 & 0.991 & 0.993 & 0.999 & 0.999 & 1.000 & 1.000 \\
\hline
\end{tabular}


Appendix Table A.4 (continued)

\begin{tabular}{|c|c|c|c|c|c|c|c|c|c|c|c|}
\hline & \multirow[b]{2}{*}{$c$} & \multicolumn{2}{|c|}{$t_{0}$} & \multicolumn{2}{|c|}{$t_{2}$} & \multicolumn{2}{|c|}{$\overline{F_{1}}$} & \multicolumn{2}{|c|}{$F_{12}$} & \multicolumn{2}{|c|}{$F_{012}$} \\
\hline & & $O L S$ & $G L S$ & $O L S$ & $G L S$ & $O L S$ & $G L S$ & $O L S$ & $G L S$ & $O L S$ & $G L S$ \\
\hline \multicolumn{12}{|l|}{ Panel B. $\ell=12$} \\
\hline \multirow[t]{3}{*}{$A I C$} & 5 & 0.086 & 0.109 & 0.122 & 0.268 & 0.203 & 0.451 & 0.280 & 0.578 & 0.257 & 0.529 \\
\hline & 10 & 0.183 & 0.296 & 0.327 & 0.657 & 0.564 & 0.866 & 0.754 & 0.971 & 0.774 & 0.970 \\
\hline & 20 & 0.643 & 0.829 & 0.885 & 0.969 & 0.993 & 0.995 & 1.000 & 1.000 & 1.000 & 1.000 \\
\hline \multirow[t]{3}{*}{$M A I C$} & 5 & 0.086 & 0.116 & 0.120 & 0.270 & 0.196 & 0.452 & 0.265 & 0.612 & 0.252 & 0.549 \\
\hline & 10 & 0.177 & 0.316 & 0.308 & 0.644 & 0.542 & 0.854 & 0.727 & 0.963 & 0.743 & 0.961 \\
\hline & 20 & 0.610 & 0.734 & 0.842 & 0.916 & 0.963 & 0.968 & 0.991 & 0.996 & 0.989 & 0.997 \\
\hline \multirow{3}{*}{$M A I C_{-} P Q$} & 5 & & 0.117 & & 0.269 & & 0.449 & & 0.608 & & 0.555 \\
\hline & 10 & & 0.321 & & 0.656 & & 0.860 & & 0.967 & & 0.967 \\
\hline & 20 & & 0.804 & & 0.948 & & 0.983 & & 0.998 & & 0.998 \\
\hline \multirow[t]{3}{*}{$B I C$} & 5 & 0.088 & 0.105 & 0.121 & 0.270 & 0.207 & 0.460 & 0.286 & 0.598 & 0.264 & 0.535 \\
\hline & 10 & 0.187 & 0.298 & 0.328 & 0.663 & 0.569 & 0.878 & 0.767 & 0.976 & 0.783 & 0.971 \\
\hline & 20 & 0.662 & 0.836 & 0.894 & 0.975 & 0.995 & 0.997 & 1.000 & 1.000 & 1.000 & 1.000 \\
\hline \multirow{3}{*}{$M B I C_{-} P Q$} & 5 & 0.085 & 0.112 & 0.120 & 0.266 & 0.205 & 0.455 & 0.277 & 0.610 & 0.264 & 0.548 \\
\hline & 10 & 0.177 & 0.314 & 0.312 & 0.658 & 0.559 & 0.874 & 0.748 & 0.977 & 0.765 & 0.974 \\
\hline & 20 & 0.612 & 0.814 & 0.859 & 0.959 & 0.977 & 0.992 & 0.995 & 0.999 & 0.995 & 0.999 \\
\hline \multirow[t]{3}{*}{$S A I C$} & 5 & 0.093 & 0.104 & 0.135 & 0.253 & 0.198 & 0.416 & 0.268 & 0.543 & 0.251 & 0.478 \\
\hline & 10 & 0.190 & 0.281 & 0.317 & 0.593 & 0.519 & 0.811 & 0.692 & 0.945 & 0.722 & 0.934 \\
\hline & 20 & 0.589 & 0.735 & 0.811 & 0.918 & 0.975 & 0.982 & 0.997 & 0.998 & 0.998 & 0.999 \\
\hline \multirow[t]{3}{*}{$S M A I C$} & 5 & 0.111 & 0.120 & 0.139 & 0.279 & 0.209 & 0.456 & 0.257 & 0.602 & 0.282 & 0.546 \\
\hline & 10 & 0.247 & 0.318 & 0.339 & 0.648 & 0.558 & 0.851 & 0.692 & 0.954 & 0.746 & 0.951 \\
\hline & 20 & 0.740 & 0.760 & 0.872 & 0.939 & 0.980 & 0.984 & 0.994 & 0.997 & 0.995 & 0.998 \\
\hline \multirow[t]{3}{*}{$S M A I C_{-} P Q$} & 5 & & 0.127 & & 0.280 & & 0.437 & & 0.596 & & 0.546 \\
\hline & 10 & & 0.342 & & 0.659 & & 0.846 & & 0.955 & & 0.951 \\
\hline & 20 & & 0.815 & & 0.946 & & 0.986 & & 0.998 & & 0.998 \\
\hline \multirow[t]{3}{*}{$t-s q(5 \%)$} & 5 & 0.084 & 0.111 & 0.129 & 0.252 & 0.186 & 0.413 & 0.261 & 0.543 & 0.241 & 0.493 \\
\hline & 10 & 0.167 & 0.266 & 0.302 & 0.589 & 0.496 & 0.789 & 0.676 & 0.931 & 0.705 & 0.929 \\
\hline & 20 & 0.515 & 0.700 & 0.777 & 0.883 & 0.938 & 0.953 & 0.987 & 0.992 & 0.992 & 0.996 \\
\hline \multirow[t]{3}{*}{$t-s q(10 \%)$} & 5 & 0.081 & 0.108 & 0.127 & 0.239 & 0.179 & 0.389 & 0.252 & 0.526 & 0.242 & 0.484 \\
\hline & 10 & 0.158 & 0.256 & 0.281 & 0.554 & 0.454 & 0.747 & 0.629 & 0.909 & 0.662 & 0.908 \\
\hline & 20 & 0.453 & 0.625 & 0.714 & 0.838 & 0.903 & 0.928 & 0.978 & 0.989 & 0.986 & 0.993 \\
\hline \multirow[t]{3}{*}{$t$-bm(10\%) } & 5 & 0.088 & 0.100 & 0.131 & 0.260 & 0.202 & 0.415 & 0.277 & 0.545 & 0.259 & 0.486 \\
\hline & 10 & 0.185 & 0.267 & 0.307 & 0.615 & 0.528 & 0.811 & 0.710 & 0.942 & 0.739 & 0.936 \\
\hline & 20 & 0.579 & 0.722 & 0.826 & 0.922 & 0.974 & 0.976 & 0.998 & 0.998 & 0.998 & 0.999 \\
\hline \multirow[t]{3}{*}{$t-r t(10 \%)$} & 5 & 0.092 & 0.101 & 0.134 & 0.249 & 0.193 & 0.405 & 0.264 & 0.547 & 0.256 & 0.471 \\
\hline & 10 & 0.193 & 0.268 & 0.310 & 0.583 & 0.509 & 0.801 & 0.682 & 0.943 & 0.724 & 0.932 \\
\hline & 20 & 0.593 & 0.718 & 0.803 & 0.912 & 0.970 & 0.979 & 0.997 & 0.998 & 0.998 & 0.999 \\
\hline
\end{tabular}

Notes: As for Table 4, except that $N=100$. 
Appendix Table A.5: Size-corrected power of quarterly sesonal unit root tests for moving average disturbances with $N=100$

\begin{tabular}{|c|c|c|c|c|c|c|c|c|c|c|c|}
\hline & \multirow[b]{2}{*}{$c$} & \multicolumn{2}{|c|}{$t_{0}$} & \multicolumn{2}{|c|}{$t_{2}$} & \multicolumn{2}{|c|}{$\overline{F_{1}}$} & \multicolumn{2}{|c|}{$F_{12}$} & \multicolumn{2}{|c|}{$F_{012}$} \\
\hline & & $O L S$ & $G L S$ & $O L S$ & $G L S$ & $O L S$ & $G L S$ & $O L S$ & $G L S$ & $O L S$ & $G L S$ \\
\hline \multicolumn{12}{|c|}{ Panel A. $M A(1): \theta=-0.8, \Theta=0$} \\
\hline \multirow[t]{3}{*}{$A I C$} & 5 & 0.079 & 0.102 & 0.187 & $\overline{0.201}$ & 0.162 & 0.375 & 0.255 & 0.389 & 0.266 & 0.405 \\
\hline & 10 & 0.174 & 0.258 & 0.405 & 0.371 & 0.475 & 0.769 & 0.630 & 0.754 & 0.704 & 0.826 \\
\hline & 20 & 0.527 & 0.671 & 0.857 & 0.557 & 0.960 & 0.954 & 0.989 & 0.930 & 0.997 & 0.980 \\
\hline \multirow[t]{3}{*}{$M A I C$} & 5 & 0.079 & 0.110 & 0.134 & 0.198 & 0.137 & 0.377 & 0.199 & 0.470 & 0.205 & 0.465 \\
\hline & 10 & 0.163 & 0.262 & 0.320 & 0.359 & 0.392 & 0.753 & 0.580 & 0.821 & 0.608 & 0.859 \\
\hline & 20 & 0.440 & 0.588 & 0.741 & 0.506 & 0.893 & 0.922 & 0.973 & 0.942 & 0.982 & 0.980 \\
\hline \multirow[t]{3}{*}{$M A I C_{-} P Q$} & 5 & & 0.113 & & $\overline{0.204}$ & & 0.375 & & 0.469 & & 0.463 \\
\hline & 10 & & 0.274 & & 0.364 & & 0.754 & & 0.818 & & 0.858 \\
\hline & 20 & & 0.633 & & 0.521 & & 0.927 & & 0.945 & & 0.983 \\
\hline \multirow[t]{3}{*}{$B I C$} & 5 & 0.082 & 0.108 & 0.172 & 0.188 & 0.170 & 0.396 & 0.239 & 0.335 & 0.257 & 0.358 \\
\hline & 10 & 0.183 & 0.272 & 0.392 & 0.361 & 0.474 & 0.787 & 0.643 & 0.664 & 0.688 & 0.740 \\
\hline & 20 & 0.544 & 0.699 & 0.886 & 0.575 & 0.953 & 0.965 & 0.988 & 0.876 & 0.995 & 0.962 \\
\hline \multirow{3}{*}{$M B I C_{-} P Q$} & 5 & 0.084 & 0.124 & 0.136 & 0.201 & 0.140 & 0.384 & 0.205 & 0.467 & 0.214 & 0.466 \\
\hline & 10 & 0.170 & 0.293 & 0.325 & 0.359 & 0.407 & 0.769 & 0.588 & 0.807 & 0.619 & 0.853 \\
\hline & 20 & 0.455 & 0.662 & 0.715 & 0.495 & 0.903 & 0.934 & 0.971 & 0.939 & 0.981 & 0.980 \\
\hline \multirow[t]{3}{*}{$\overline{S A I C}$} & 5 & 0.083 & 0.099 & 0.165 & 0.186 & 0.149 & 0.353 & 0.237 & 0.390 & 0.247 & 0.401 \\
\hline & 10 & 0.168 & 0.236 & 0.380 & 0.344 & 0.414 & 0.735 & 0.619 & 0.744 & 0.666 & 0.804 \\
\hline & 20 & 0.472 & 0.565 & 0.828 & 0.517 & 0.918 & 0.921 & 0.982 & 0.909 & 0.991 & 0.964 \\
\hline \multirow[t]{3}{*}{$S M A I C$} & 5 & 0.107 & 0.118 & 0.139 & 0.202 & 0.153 & 0.386 & 0.236 & 0.466 & 0.257 & 0.457 \\
\hline & 10 & 0.217 & 0.280 & 0.321 & 0.347 & 0.412 & 0.765 & 0.627 & 0.818 & 0.684 & 0.859 \\
\hline & 20 & 0.518 & 0.616 & 0.757 & 0.500 & 0.904 & 0.923 & 0.984 & 0.943 & 0.992 & 0.982 \\
\hline \multirow[t]{3}{*}{$S M A I C \_P Q$} & 5 & & 0.123 & & 0.200 & & 0.378 & & 0.457 & & 0.457 \\
\hline & 10 & & 0.290 & & 0.342 & & 0.756 & & 0.809 & & 0.859 \\
\hline & 20 & & 0.628 & & 0.511 & & 0.923 & & 0.938 & & 0.982 \\
\hline \multirow[t]{3}{*}{$t-s q(5 \%)$} & 5 & 0.077 & 0.098 & 0.152 & 0.173 & 0.154 & 0.373 & 0.236 & 0.395 & 0.229 & 0.403 \\
\hline & 10 & 0.167 & 0.242 & 0.321 & 0.323 & 0.428 & 0.749 & 0.593 & 0.742 & 0.628 & 0.803 \\
\hline & 20 & 0.459 & 0.577 & 0.704 & 0.482 & 0.915 & 0.919 & 0.967 & 0.906 & 0.980 & 0.965 \\
\hline \multirow[t]{3}{*}{$t-s q(10 \%)$} & 5 & 0.083 & 0.100 & 0.133 & 0.192 & 0.147 & 0.366 & 0.208 & 0.415 & 0.221 & 0.412 \\
\hline & 10 & 0.160 & 0.236 & 0.302 & 0.336 & 0.403 & 0.741 & 0.581 & 0.774 & 0.630 & 0.813 \\
\hline & 20 & 0.420 & 0.535 & 0.717 & 0.492 & 0.886 & 0.911 & 0.971 & 0.918 & 0.981 & 0.969 \\
\hline \multirow[t]{3}{*}{$t$-bm(10\%) } & 5 & 0.082 & 0.105 & 0.166 & 0.188 & 0.156 & 0.360 & 0.228 & 0.405 & 0.237 & 0.424 \\
\hline & 10 & 0.164 & 0.244 & 0.385 & 0.346 & 0.438 & 0.746 & 0.627 & 0.764 & 0.672 & 0.828 \\
\hline & 20 & 0.485 & 0.582 & 0.852 & 0.503 & 0.942 & 0.929 & 0.985 & 0.910 & 0.991 & 0.968 \\
\hline \multirow[t]{3}{*}{$t-r t(10 \%)$} & 5 & 0.081 & 0.101 & 0.161 & 0.187 & 0.156 & 0.347 & 0.237 & 0.392 & 0.243 & 0.404 \\
\hline & 10 & 0.164 & 0.237 & 0.377 & 0.349 & 0.418 & 0.726 & 0.620 & 0.744 & 0.662 & 0.809 \\
\hline & 20 & 0.463 & 0.565 & 0.821 & 0.517 & 0.919 & 0.914 & 0.981 & 0.908 & 0.991 & 0.965 \\
\hline
\end{tabular}


Appendix Table A.5 (continued)

\begin{tabular}{|c|c|c|c|c|c|c|c|c|c|c|c|}
\hline & \multirow[b]{2}{*}{$c$} & \multicolumn{2}{|c|}{$t_{0}$} & \multicolumn{2}{|c|}{$t_{2}$} & \multicolumn{2}{|c|}{$\overline{F_{1}}$} & \multicolumn{2}{|c|}{$F_{12}$} & \multicolumn{2}{|c|}{$F_{012}$} \\
\hline & & $O L S$ & $G L S$ & $O L S$ & $G L S$ & $O L S$ & $G L S$ & $O L S$ & $G L S$ & $O L S$ & $G L S$ \\
\hline \multicolumn{12}{|c|}{ Panel B. Seasonal $M A: \theta=0, \Theta=0.5$} \\
\hline \multirow[t]{3}{*}{$A I C$} & 5 & 0.112 & 0.111 & 0.172 & 0.249 & 0.304 & 0.434 & 0.401 & 0.504 & 0.372 & 0.443 \\
\hline & 10 & 0.311 & 0.270 & 0.473 & 0.507 & 0.744 & 0.695 & 0.840 & 0.792 & 0.826 & 0.764 \\
\hline & 20 & 0.827 & 0.617 & 0.942 & 0.717 & 0.991 & 0.798 & 0.997 & 0.897 & 0.997 & 0.922 \\
\hline \multirow[t]{3}{*}{$M A I C$} & 5 & 0.077 & 0.080 & 0.133 & 0.210 & 0.196 & 0.379 & 0.250 & 0.467 & 0.229 & 0.400 \\
\hline & 10 & 0.180 & 0.174 & 0.328 & 0.426 & 0.544 & 0.599 & 0.658 & 0.750 & 0.637 & 0.731 \\
\hline & 20 & 0.523 & 0.426 & 0.768 & 0.615 & 0.952 & 0.701 & 0.987 & 0.871 & 0.988 & 0.907 \\
\hline \multirow[t]{3}{*}{$M A I C_{-} P Q$} & 5 & & 0.086 & & 0.216 & & 0.385 & & 0.476 & & 0.383 \\
\hline & 10 & & 0.205 & & 0.456 & & 0.622 & & 0.764 & & 0.739 \\
\hline & 20 & & 0.524 & & 0.677 & & 0.755 & & 0.900 & & 0.927 \\
\hline \multirow[t]{3}{*}{$B I C$} & 5 & 0.217 & 0.139 & 0.273 & 0.257 & 0.344 & 0.478 & 0.354 & 0.588 & 0.314 & 0.515 \\
\hline & 10 & 0.554 & 0.365 & 0.647 & 0.568 & 0.758 & 0.772 & 0.802 & 0.896 & 0.754 & 0.895 \\
\hline & 20 & 0.972 & 0.760 & 0.986 & 0.822 & 0.999 & 0.901 & 1.000 & 0.973 & 1.000 & 0.983 \\
\hline \multirow[t]{3}{*}{$M B I C_{-} P Q$} & 5 & 0.065 & 0.080 & 0.118 & 0.199 & 0.203 & 0.390 & 0.249 & 0.469 & 0.206 & 0.364 \\
\hline & 10 & 0.148 & 0.175 & 0.289 & 0.416 & 0.544 & 0.623 & 0.652 & 0.748 & 0.591 & 0.706 \\
\hline & 20 & 0.466 & 0.469 & 0.728 & 0.636 & 0.947 & 0.751 & 0.984 & 0.884 & 0.975 & 0.904 \\
\hline \multirow[t]{3}{*}{$S A I C$} & 5 & 0.131 & 0.128 & 0.194 & 0.254 & 0.293 & 0.400 & 0.334 & 0.483 & 0.293 & 0.395 \\
\hline & 10 & 0.368 & 0.314 & 0.508 & 0.532 & 0.721 & 0.661 & 0.790 & 0.779 & 0.740 & 0.722 \\
\hline & 20 & 0.873 & 0.620 & 0.950 & 0.729 & 0.989 & 0.769 & 0.997 & 0.893 & 0.991 & 0.872 \\
\hline \multirow[t]{3}{*}{$S M A I C$} & 5 & 0.184 & 0.151 & 0.202 & 0.267 & 0.270 & 0.433 & 0.323 & 0.526 & 0.289 & 0.476 \\
\hline & 10 & 0.506 & 0.358 & 0.578 & 0.566 & 0.727 & 0.718 & 0.791 & 0.822 & 0.759 & 0.808 \\
\hline & 20 & 0.962 & 0.647 & 0.984 & 0.780 & 0.996 & 0.852 & 0.999 & 0.928 & 0.998 & 0.942 \\
\hline \multirow[t]{3}{*}{$S M A I C \_P Q$} & 5 & & 0.155 & & 0.276 & & 0.424 & & 0.526 & & 0.476 \\
\hline & 10 & & 0.388 & & 0.582 & & 0.709 & & 0.827 & & 0.808 \\
\hline & 20 & & 0.731 & & 0.802 & & 0.853 & & 0.938 & & 0.942 \\
\hline \multirow[t]{3}{*}{$t-s q(5 \%)$} & 5 & 0.105 & 0.100 & 0.159 & 0.219 & 0.250 & 0.379 & 0.305 & 0.462 & 0.289 & 0.406 \\
\hline & 10 & 0.271 & 0.226 & 0.411 & 0.457 & 0.630 & 0.617 & 0.731 & 0.757 & 0.725 & 0.742 \\
\hline & 20 & 0.680 & 0.492 & 0.840 & 0.638 & 0.967 & 0.715 & 0.990 & 0.865 & 0.988 & 0.899 \\
\hline \multirow[t]{3}{*}{$t-s q(10 \%)$} & 5 & 0.097 & 0.099 & 0.148 & 0.227 & 0.224 & 0.356 & 0.281 & 0.446 & 0.274 & 0.389 \\
\hline & 10 & 0.232 & 0.216 & 0.371 & 0.461 & 0.595 & 0.584 & 0.715 & 0.740 & 0.724 & 0.735 \\
\hline & 20 & 0.609 & 0.463 & 0.805 & 0.638 & 0.959 & 0.681 & 0.991 & 0.855 & 0.990 & 0.894 \\
\hline \multirow[t]{3}{*}{$t-b m(10 \%)$} & 5 & 0.138 & 0.128 & 0.200 & 0.256 & 0.303 & 0.396 & 0.337 & 0.476 & 0.300 & 0.398 \\
\hline & 10 & 0.379 & 0.315 & 0.535 & 0.541 & 0.738 & 0.656 & 0.801 & 0.775 & 0.750 & 0.726 \\
\hline & 20 & 0.895 & 0.637 & 0.959 & 0.737 & 0.993 & 0.759 & 0.998 & 0.890 & 0.992 & 0.882 \\
\hline \multirow[t]{3}{*}{$\overline{t-r t(10 \%)}$} & 5 & 0.135 & 0.126 & 0.191 & 0.256 & 0.290 & 0.396 & 0.331 & 0.479 & 0.290 & 0.389 \\
\hline & 10 & 0.368 & 0.312 & 0.507 & 0.532 & 0.719 & 0.652 & 0.788 & 0.774 & 0.735 & 0.716 \\
\hline & 20 & 0.865 & 0.617 & 0.945 & 0.730 & 0.988 & 0.760 & 0.997 & 0.891 & 0.991 & 0.869 \\
\hline
\end{tabular}


Appendix Table A.5 (continued)

\begin{tabular}{|c|c|c|c|c|c|c|c|c|c|c|c|}
\hline & \multirow[b]{2}{*}{$c$} & \multicolumn{2}{|c|}{$t_{0}$} & \multicolumn{2}{|c|}{$t_{2}$} & \multicolumn{2}{|c|}{$\overline{F_{1}}$} & \multicolumn{2}{|c|}{$F_{12}$} & \multicolumn{2}{|c|}{$F_{012}$} \\
\hline & & $O L S$ & $G L S$ & $O L S$ & $G L S$ & $O L S$ & $G L S$ & $O L S$ & $G L S$ & $O L S$ & $G L S$ \\
\hline \multicolumn{12}{|c|}{ Panel C. Seasonal $M A: \theta=0, \Theta=-0.5$} \\
\hline \multirow[t]{3}{*}{$A I C$} & 5 & 0.085 & 0.118 & 0.106 & 0.250 & 0.178 & 0.373 & 0.219 & 0.490 & 0.220 & 0.429 \\
\hline & 10 & 0.164 & 0.242 & 0.234 & 0.539 & 0.422 & 0.743 & 0.532 & 0.870 & 0.530 & 0.836 \\
\hline & 20 & 0.432 & 0.568 & 0.607 & 0.864 & 0.847 & 0.958 & 0.936 & 0.996 & 0.949 & 0.998 \\
\hline \multirow[t]{3}{*}{$M A I C$} & 5 & 0.069 & $\begin{array}{l}0.103 \\
\end{array}$ & 0.079 & 0.260 & 0.122 & 0.360 & 0.165 & 0.505 & 0.161 & 0.444 \\
\hline & 10 & 0.129 & 0.238 & 0.188 & 0.564 & 0.341 & 0.751 & 0.480 & 0.908 & 0.501 & 0.897 \\
\hline & 20 & 0.352 & 0.601 & 0.535 & 0.885 & 0.833 & 0.966 & 0.945 & 0.997 & 0.963 & 0.998 \\
\hline \multirow[t]{3}{*}{$M A I C_{-} P Q$} & 5 & & 0.107 & & 0.257 & & 0.354 & & 0.506 & & 0.442 \\
\hline & 10 & & 0.255 & & 0.563 & & 0.747 & & 0.910 & & 0.897 \\
\hline & 20 & & 0.605 & & 0.886 & & 0.966 & & 0.998 & & 0.998 \\
\hline \multirow[t]{3}{*}{$B I C$} & 5 & 0.090 & 0.113 & 0.134 & 0.268 & 0.239 & 0.529 & 0.297 & 0.644 & 0.283 & 0.561 \\
\hline & 10 & 0.180 & 0.271 & 0.307 & 0.599 & 0.606 & 0.898 & 0.734 & 0.950 & 0.743 & 0.934 \\
\hline & 20 & 0.538 & 0.684 & 0.769 & 0.918 & 0.967 & 0.989 & 0.986 & 0.998 & 0.984 & 0.996 \\
\hline \multirow[t]{3}{*}{$M B I C_{-} P Q$} & 5 & 0.027 & 0.039 & 0.031 & 0.170 & 0.067 & 0.262 & 0.070 & 0.350 & 0.052 & 0.267 \\
\hline & 10 & 0.037 & 0.096 & 0.064 & 0.389 & 0.170 & 0.582 & 0.206 & 0.777 & 0.173 & 0.720 \\
\hline & 20 & 0.197 & 0.460 & 0.358 & 0.882 & 0.718 & 0.968 & 0.879 & 0.998 & 0.906 & 0.999 \\
\hline \multirow[t]{3}{*}{$S A I C$} & 5 & 0.083 & 0.100 & 0.128 & 0.248 & 0.180 & 0.383 & 0.219 & 0.479 & 0.195 & 0.390 \\
\hline & 10 & 0.168 & 0.214 & 0.282 & 0.534 & 0.438 & 0.766 & 0.549 & 0.873 & 0.509 & 0.819 \\
\hline & 20 & 0.447 & 0.551 & 0.676 & 0.872 & 0.886 & 0.957 & 0.953 & 0.993 & 0.942 & 0.989 \\
\hline \multirow[t]{3}{*}{$S M A I C$} & 5 & 0.093 & 0.115 & 0.103 & 0.273 & 0.166 & 0.416 & 0.194 & 0.552 & 0.210 & 0.473 \\
\hline & 10 & 0.199 & 0.275 & 0.246 & 0.597 & 0.442 & 0.812 & 0.534 & 0.923 & 0.557 & 0.887 \\
\hline & 20 & 0.372 & 0.600 & 0.479 & 0.867 & 0.685 & 0.948 & 0.718 & 0.973 & 0.710 & 0.845 \\
\hline \multirow[t]{3}{*}{$S M A I C \_P Q$} & 5 & & 0.121 & & 0.277 & & 0.399 & & 0.554 & & 0.473 \\
\hline & 10 & & 0.298 & & 0.607 & & 0.797 & & 0.924 & & 0.887 \\
\hline & 20 & & 0.503 & & 0.747 & & 0.855 & & 0.898 & & 0.845 \\
\hline \multirow[t]{3}{*}{$t-s q(5 \%)$} & 5 & 0.077 & 0.100 & 0.101 & 0.268 & 0.158 & 0.360 & 0.210 & 0.487 & 0.196 & 0.443 \\
\hline & 10 & 0.141 & 0.211 & 0.213 & 0.546 & 0.383 & 0.722 & 0.519 & 0.877 & 0.523 & 0.860 \\
\hline & 20 & 0.372 & 0.512 & 0.546 & 0.850 & 0.835 & 0.944 & 0.942 & 0.990 & 0.952 & 0.994 \\
\hline \multirow[t]{3}{*}{$t-s q(10 \%)$} & 5 & 0.078 & 0.096 & 0.100 & 0.263 & 0.160 & 0.373 & 0.209 & 0.484 & 0.197 & 0.437 \\
\hline & 10 & 0.139 & 0.205 & 0.212 & 0.531 & 0.384 & 0.727 & 0.523 & 0.873 & 0.528 & 0.856 \\
\hline & 20 & 0.360 & 0.489 & 0.528 & 0.832 & 0.827 & 0.939 & 0.936 & 0.988 & 0.950 & 0.992 \\
\hline \multirow[t]{3}{*}{$t-b m(10 \%)$} & 5 & 0.085 & 0.104 & 0.125 & 0.246 & 0.184 & 0.383 & 0.218 & 0.507 & 0.195 & 0.415 \\
\hline & 10 & 0.170 & 0.222 & 0.283 & 0.536 & 0.456 & 0.757 & 0.554 & 0.887 & 0.523 & 0.841 \\
\hline & 20 & 0.471 & 0.562 & 0.682 & 0.871 & 0.891 & 0.957 & 0.954 & 0.993 & 0.946 & 0.989 \\
\hline \multirow[t]{3}{*}{$\overline{t-r t(10 \%)}$} & 5 & 0.084 & 0.101 & 0.130 & 0.250 & 0.183 & 0.383 & 0.218 & 0.477 & 0.195 & 0.385 \\
\hline & 10 & 0.169 & 0.215 & 0.283 & 0.534 & 0.444 & 0.762 & 0.543 & 0.871 & 0.509 & 0.812 \\
\hline & 20 & 0.442 & 0.548 & 0.677 & 0.874 & 0.885 & 0.956 & 0.951 & 0.992 & 0.941 & 0.988 \\
\hline
\end{tabular}

Notes: As for Table 5, except that $N=100$. 
Appendix Table A.6: Size-corrected power of quarterly seasonal unit root tests for autoregressive disturbances with $N=100$

\begin{tabular}{|c|c|c|c|c|c|c|c|c|c|c|c|}
\hline & \multirow[b]{2}{*}{$c$} & \multicolumn{2}{|c|}{$t_{0}$} & \multicolumn{2}{|c|}{$t_{2}$} & \multicolumn{2}{|c|}{$\overline{F_{1}}$} & \multicolumn{2}{|c|}{$F_{12}$} & \multicolumn{2}{|c|}{$F_{012}$} \\
\hline & & $O L S$ & $G L S$ & $O L S$ & $G L S$ & $O L S$ & $G L S$ & $O L S$ & $G L S$ & $O L S$ & $G L S$ \\
\hline \multicolumn{12}{|c|}{ A. Seasonal $A R: \phi=0, \Phi=0.5$} \\
\hline \multirow[t]{3}{*}{$A I C$} & 5 & 0.087 & 0.105 & $\overline{0.110}$ & 0.274 & 0.150 & 0.434 & 0.196 & 0.576 & 0.208 & 0.502 \\
\hline & 10 & 0.157 & 0.245 & 0.249 & 0.581 & 0.423 & 0.839 & 0.573 & 0.950 & 0.603 & 0.938 \\
\hline & 20 & 0.426 & 0.595 & 0.626 & 0.910 & 0.886 & 0.994 & 0.971 & 0.999 & 0.986 & 0.999 \\
\hline \multirow[t]{3}{*}{$M A I C$} & 5 & 0.088 & 0.110 & 0.103 & 0.270 & 0.133 & 0.440 & 0.195 & 0.587 & 0.198 & 0.532 \\
\hline & 10 & 0.157 & 0.250 & 0.244 & 0.577 & 0.395 & 0.841 & 0.564 & 0.946 & 0.571 & 0.937 \\
\hline & 20 & 0.356 & 0.561 & 0.544 & 0.871 & 0.787 & 0.982 & 0.901 & 0.997 & 0.901 & 0.997 \\
\hline \multirow[t]{3}{*}{$\overline{M A I C_{-} P Q}$} & 5 & & 0.112 & & 0.278 & & $\overline{0.440}$ & & 0.593 & & 0.535 \\
\hline & 10 & & 0.258 & & 0.594 & & 0.843 & & 0.948 & & 0.940 \\
\hline & 20 & & 0.548 & & 0.865 & & 0.976 & & 0.995 & & 0.996 \\
\hline \multirow[t]{3}{*}{$B I C$} & 5 & 0.087 & 0.108 & 0.108 & 0.280 & 0.147 & 0.444 & 0.203 & 0.588 & 0.213 & 0.513 \\
\hline & 10 & 0.160 & 0.250 & 0.256 & 0.593 & 0.423 & 0.848 & 0.593 & 0.956 & 0.611 & 0.942 \\
\hline & 20 & 0.435 & 0.609 & 0.639 & 0.918 & 0.897 & 0.995 & 0.975 & 0.999 & 0.987 & 0.999 \\
\hline \multirow{3}{*}{$M B I C_{-} P Q$} & 5 & 0.032 & 0.049 & 0.048 & 0.172 & 0.082 & 0.318 & 0.095 & 0.404 & 0.075 & 0.343 \\
\hline & 10 & 0.011 & 0.022 & 0.022 & 0.114 & 0.093 & 0.359 & 0.096 & 0.383 & 0.067 & 0.262 \\
\hline & 20 & 0.016 & 0.039 & 0.058 & 0.335 & 0.225 & 0.749 & 0.273 & 0.883 & 0.182 & 0.810 \\
\hline \multirow[t]{3}{*}{$\overline{S A I C}$} & 5 & 0.085 & 0.093 & 0.114 & 0.247 & 0.155 & 0.381 & 0.203 & 0.518 & 0.197 & 0.434 \\
\hline & 10 & 0.152 & 0.214 & 0.250 & 0.533 & 0.405 & 0.777 & 0.546 & 0.915 & 0.545 & 0.886 \\
\hline & 20 & 0.383 & 0.535 & 0.595 & 0.863 & 0.839 & 0.977 & 0.946 & 0.996 & 0.955 & 0.994 \\
\hline \multirow[t]{3}{*}{$S M A I C$} & 5 & 0.108 & 0.122 & 0.117 & 0.247 & 0.177 & 0.462 & 0.231 & 0.603 & 0.241 & 0.557 \\
\hline & 10 & 0.205 & 0.289 & 0.285 & 0.586 & 0.489 & 0.854 & 0.618 & 0.950 & 0.623 & 0.932 \\
\hline & 20 & 0.459 & 0.649 & 0.606 & 0.884 & 0.816 & 0.985 & 0.873 & 0.993 & 0.870 & 0.959 \\
\hline \multirow[t]{3}{*}{$S M A I C_{-} P Q$} & 5 & & 0.127 & & 0.251 & & 0.461 & & 0.594 & & 0.557 \\
\hline & 10 & & 0.303 & & 0.589 & & 0.855 & & 0.947 & & 0.932 \\
\hline & 20 & & 0.626 & & 0.846 & & 0.956 & & 0.972 & & 0.959 \\
\hline \multirow[t]{3}{*}{$t-s q(5 \%)$} & 5 & 0.084 & 0.104 & 0.114 & 0.265 & 0.148 & 0.405 & 0.200 & 0.549 & 0.206 & 0.482 \\
\hline & 10 & 0.150 & 0.232 & 0.238 & 0.551 & 0.401 & 0.792 & 0.556 & 0.924 & 0.571 & 0.914 \\
\hline & 20 & 0.384 & 0.543 & 0.572 & 0.867 & 0.828 & 0.975 & 0.942 & 0.997 & 0.960 & 0.997 \\
\hline \multirow[t]{3}{*}{$t-s q(10 \%)$} & 5 & 0.080 & 0.099 & 0.108 & 0.252 & 0.142 & 0.382 & 0.197 & 0.525 & 0.190 & 0.446 \\
\hline & 10 & 0.140 & 0.221 & 0.223 & 0.536 & 0.377 & 0.760 & 0.542 & 0.903 & 0.541 & 0.886 \\
\hline & 20 & 0.348 & 0.498 & 0.526 & 0.834 & 0.784 & 0.962 & 0.923 & 0.995 & 0.937 & 0.994 \\
\hline \multirow[t]{3}{*}{$t$-bm(10\%) } & 5 & 0.101 & 0.104 & 0.119 & 0.257 & 0.160 & 0.390 & 0.217 & 0.523 & 0.211 & 0.469 \\
\hline & 10 & 0.164 & 0.225 & 0.258 & 0.549 & 0.420 & 0.781 & 0.581 & 0.916 & 0.579 & 0.903 \\
\hline & 20 & 0.423 & 0.564 & 0.614 & 0.864 & 0.849 & 0.978 & 0.953 & 0.997 & 0.960 & 0.996 \\
\hline \multirow[t]{3}{*}{$t-r t(10 \%)$} & 5 & 0.091 & 0.089 & 0.114 & 0.245 & 0.154 & 0.379 & 0.203 & 0.518 & 0.197 & 0.432 \\
\hline & 10 & 0.159 & 0.210 & 0.248 & 0.527 & 0.404 & 0.765 & 0.545 & 0.909 & 0.544 & 0.884 \\
\hline & 20 & 0.394 & 0.516 & 0.588 & 0.852 & 0.829 & 0.974 & 0.943 & 0.996 & 0.953 & 0.994 \\
\hline
\end{tabular}


Appendix Table A.6 (continued)

\begin{tabular}{|c|c|c|c|c|c|c|c|c|c|c|c|}
\hline & \multirow[b]{2}{*}{$c$} & \multicolumn{2}{|c|}{$t_{0}$} & \multicolumn{2}{|c|}{$t_{2}$} & \multicolumn{2}{|c|}{$\overline{F_{1}}$} & \multicolumn{2}{|c|}{$F_{12}$} & \multicolumn{2}{|c|}{$F_{012}$} \\
\hline & & $O L S$ & $G L S$ & $O L S$ & $G L S$ & $O L S$ & $G L S$ & $O L S$ & $G L S$ & $O L S$ & $G L S$ \\
\hline \multicolumn{12}{|c|}{ Panel B. Seasonal $A R: \phi=0, \Phi=-0.5$} \\
\hline \multirow[t]{3}{*}{$A I C$} & 5 & 0.088 & 0.124 & 0.122 & 0.265 & 0.159 & 0.340 & 0.212 & 0.496 & 0.234 & 0.474 \\
\hline & 10 & 0.186 & 0.273 & 0.318 & 0.554 & 0.485 & 0.681 & 0.678 & 0.873 & 0.729 & 0.900 \\
\hline & 20 & 0.627 & 0.739 & 0.842 & 0.864 & 0.980 & 0.889 & 0.999 & 0.989 & 1.000 & 0.997 \\
\hline \multirow[t]{3}{*}{$M A I C$} & 5 & 0.090 & 0.121 & 0.119 & 0.258 & 0.153 & 0.343 & 0.195 & 0.492 & 0.221 & 0.475 \\
\hline & 10 & 0.183 & 0.268 & 0.305 & 0.538 & 0.467 & 0.666 & 0.636 & 0.849 & 0.688 & 0.869 \\
\hline & 20 & 0.591 & 0.608 & 0.798 & 0.786 & 0.955 & 0.819 & 0.987 & 0.948 & 0.988 & 0.971 \\
\hline \multirow[t]{3}{*}{$M A I C_{-} P Q$} & 5 & & 0.130 & & 0.265 & & 0.333 & & 0.492 & & 0.478 \\
\hline & 10 & & 0.287 & & 0.551 & & 0.662 & & 0.855 & & 0.876 \\
\hline & 20 & & 0.711 & & 0.834 & & 0.855 & & 0.967 & & 0.983 \\
\hline \multirow[t]{3}{*}{$B I C$} & 5 & 0.094 & 0.125 & 0.126 & 0.270 & 0.158 & 0.346 & 0.217 & 0.498 & 0.235 & 0.477 \\
\hline & 10 & 0.195 & 0.281 & 0.321 & 0.563 & 0.489 & 0.689 & 0.689 & 0.877 & 0.733 & 0.903 \\
\hline & 20 & 0.650 & 0.751 & 0.862 & 0.877 & 0.985 & 0.903 & 0.999 & 0.991 & 1.000 & 0.998 \\
\hline \multirow[t]{3}{*}{$M B I C_{-} P Q$} & 5 & 0.095 & 0.128 & 0.120 & 0.265 & 0.155 & 0.344 & 0.205 & 0.497 & 0.223 & 0.484 \\
\hline & 10 & 0.183 & 0.282 & 0.310 & 0.552 & 0.476 & 0.676 & 0.655 & 0.861 & 0.703 & 0.891 \\
\hline & 20 & 0.606 & 0.710 & 0.814 & 0.840 & 0.959 & 0.866 & 0.989 & 0.972 & 0.990 & 0.986 \\
\hline \multirow[t]{3}{*}{$S A I C$} & 5 & 0.106 & 0.124 & 0.138 & 0.266 & 0.187 & 0.357 & 0.216 & 0.481 & 0.236 & 0.438 \\
\hline & 10 & 0.236 & 0.284 & 0.373 & 0.563 & 0.545 & 0.690 & 0.686 & 0.849 & 0.711 & 0.852 \\
\hline & 20 & 0.740 & 0.714 & 0.883 & 0.852 & 0.974 & 0.861 & 0.997 & 0.968 & 0.998 & 0.977 \\
\hline \multirow[t]{3}{*}{$S M A I C$} & 5 & 0.125 & 0.144 & 0.152 & 0.290 & 0.186 & 0.405 & 0.232 & 0.530 & 0.249 & 0.493 \\
\hline & 10 & 0.329 & 0.338 & 0.423 & 0.596 & 0.590 & 0.740 & 0.715 & 0.865 & 0.727 & 0.878 \\
\hline & 20 & 0.875 & 0.709 & 0.947 & 0.859 & 0.989 & 0.894 & 0.997 & 0.960 & 0.997 & 0.980 \\
\hline \multirow[t]{3}{*}{$S M A I C \_P Q$} & 5 & & 0.152 & & 0.297 & & 0.398 & & 0.524 & & 0.493 \\
\hline & 10 & & 0.363 & & 0.618 & & 0.737 & & 0.871 & & 0.878 \\
\hline & 20 & & 0.795 & & 0.884 & & 0.911 & & 0.977 & & 0.980 \\
\hline \multirow[t]{3}{*}{$t-s q(5 \%)$} & 5 & 0.083 & 0.110 & 0.120 & 0.246 & 0.156 & 0.317 & 0.198 & 0.469 & 0.231 & 0.440 \\
\hline & 10 & 0.173 & 0.243 & 0.308 & 0.499 & 0.457 & 0.625 & 0.622 & 0.822 & 0.693 & 0.850 \\
\hline & 20 & 0.554 & 0.617 & 0.779 & 0.787 & 0.947 & 0.804 & 0.989 & 0.948 & 0.995 & 0.976 \\
\hline \multirow[t]{3}{*}{$t-s q(10 \%)$} & 5 & 0.083 & 0.107 & 0.120 & 0.228 & 0.155 & 0.309 & 0.193 & 0.444 & 0.220 & 0.416 \\
\hline & 10 & 0.166 & 0.232 & 0.292 & 0.458 & 0.431 & 0.599 & 0.589 & 0.790 & 0.659 & 0.815 \\
\hline & 20 & 0.502 & 0.559 & 0.729 & 0.736 & 0.922 & 0.759 & 0.983 & 0.925 & 0.991 & 0.962 \\
\hline \multirow[t]{3}{*}{$t$-bm $(10 \%)$} & 5 & 0.102 & 0.134 & 0.141 & 0.268 & 0.194 & 0.341 & 0.218 & 0.476 & 0.233 & 0.436 \\
\hline & 10 & 0.231 & 0.294 & 0.376 & 0.561 & 0.563 & 0.672 & 0.692 & 0.849 & 0.704 & 0.854 \\
\hline & 20 & 0.741 & 0.730 & 0.891 & 0.849 & 0.980 & 0.848 & 0.999 & 0.965 & 0.999 & 0.974 \\
\hline \multirow[t]{3}{*}{$\overline{t-r t(10 \%)}$} & 5 & 0.110 & 0.120 & 0.139 & 0.268 & 0.187 & 0.353 & 0.214 & 0.485 & 0.229 & 0.435 \\
\hline & 10 & 0.238 & 0.273 & 0.369 & 0.558 & 0.541 & 0.683 & 0.686 & 0.848 & 0.697 & 0.850 \\
\hline & 20 & 0.734 & 0.701 & 0.878 & 0.846 & 0.972 & 0.853 & 0.997 & 0.966 & 0.998 & 0.975 \\
\hline
\end{tabular}

Notes: As for Table 6, except that $N=100$. 